\title{
Quantum Channels Arising From Abstract Harmonic Analysis
}

by

\author{
Jason A. Crann \\ A thesis submitted to \\ the Faculty of Graduate and Postdoctoral Affairs \\ in partial fulfillment of \\ the requirements for the degree of \\ Master of Science
}

School of Mathematics and Statistics Ottawa-Carleton Institute for Mathematics and Statistics Carleton University Ottawa, Ontario, Canada

(C)Copyright 2011, Jason A. Crann 
Library and Archives

Canada

Published Heritage

Branch

395 Wellington Street Ottawa ON K1A ON4 Canada
Bibliothèque et

Archives Canada

Direction du

Patrimoine de l'édition

395, rue Wellington

Ottawa ON K1A ON4

Canada
Your file Votre référence

ISBN: 978-0-494-83171-7

Our file Notre référence

ISBN: $978-0-494-83171-7$
NOTICE:

The author has granted a nonexclusive license allowing Library and Archives Canada to reproduce, publish, archive, preserve, conserve, communicate to the public by telecommunication or on the Internet, loan, distribute and sell theses worldwide, for commercial or noncommercial purposes, in microform, paper, electronic and/or any other formats.

The author retains copyright ownership and moral rights in this thesis. Neither the thesis nor substantial extracts from it may be printed or otherwise reproduced without the author's permission.

\section{AVIS:}

L'auteur a accordé une licence non exclusive permettant à la Bibliothèque et Archives Canada de reproduire, publier, archiver, sauvegarder, conserver, transmettre au public par télécommunication ou par l'Internet, prêter, distribuer et vendre des thèses partout dans le monde, à des fins commerciales ou autres, sur support microforme, papier, électronique et/ou autres formats.

L'auteur conserve la propriété du droit d'auteur et des droits moraux qui protège cette thèse. $\mathrm{Ni}$ la thèse ni des extraits substantiels de celle-ci ne doivent être imprimés ou autrement reproduits sans son autorisation.
In compliance with the Canadian Privacy Act some supporting forms may have been removed from this thesis.

While these forms may be included in the document page count, their removal does not represent any loss of content from the thesis.
Conformément à la loi canadienne sur la protection de la vie privée, quelques formulaires secondaires ont été enlevés de cette thèse.

Bien que ces formulaires aient inclus dans la pagination, il n'y aura aucun contenu manquant. 


\section{Abstract}

Throughout the $20^{\text {th }}$ century, harmonic analysis played a critical role in the theoretical development of quantum mechanics and the standard model of particle physics. Today, it continues to provide important applications to many areas of modern science. This thesis is dedicated to a new application of harmonic analysis to the growing field of quantum information. One of the central mathematical objects in quantum information is the notion of a quantum channel, the quantum analogue of a classical information channel. Using the recent representation theory of quantum groups, we present a systematic method to generate an intriguing class of quantum channels for every locally compact quantum group. Moreover, we obtain explicit Kraus decompositions for these channels, which are required if one hopes to implement them in the laboratory. In the group setting, we not only recover well known channels of importance in quantum error correction, we obtain new counter-examples to recently solved conjectures. The fixed points of these quantum group channels - a highly sought-after object - have recently been characterized, and we explicitly compute them for the smallest nontrivial quantum group. 


\section{Acknowledgements}

First and foremost I would like to thank my supervisor, Dr. Matthias Neufang, for providing invaluable guidance and support throughout the project. His teachings have opened my eyes to many new areas of mathematics and strengthened by passion for the subject as a whole. I am really looking forward to working with him in the future.

A special thanks also goes out to Dr. Mehrdad Kalantar, whose guidance in the early stages of the Masters was crucial to my success. He was truly a co-supervisor to this project.

Finally, I would like to thank my friends and family whose love and support are responsible for the man I am today. In particular, this thesis would not have been possible without the reassuring love of my beautiful fiancée, Carley. 


\section{Contents}

$\begin{array}{ll}\text { Abstract } & \text { ii }\end{array}$

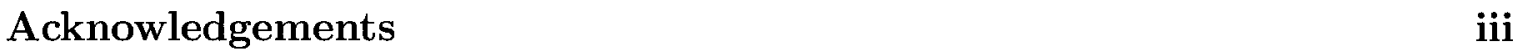

1 Introduction $\quad 1$

2 Preliminaries $\quad 4$

2.1 Topological Groups . . . . . . . . . . . . . . . . . 4

2.2 Banach Algebras . . . . . . . . . . . . . . . . 7

2.3 Operator Spaces . . . . . . . . . . . . . . . . 14

2.4 von Neumann Algebras . . . . . . . . . . . . . . . . . . 17

2.5 Quantum Information Theory ............... 25

3 Locally Compact Quantum Groups $\quad 31$

3.1 von Neumann Algebraic Quantum Groups . . . . . . . . . . . . 32

3.2 The Fundamental Unitary . . . . . . . . . . . . . . . . 37

3.3 Duality............................... 40 
3.4 The Antipode . . . . . . . . . . . . . . . . . . 43

3.5 Quantum Groups in the Universal Setting . . . . . . . . . . . . 46

4 Completely Bounded Multipliers $\quad 52$

4.1 Introduction . . . . . . . . . . . . . . . . 52

4.2 The Representation Theorem . . . . . . . . . . . . . . . 57

4.3 Positive Definite Functionals . . . . . . . . . . . . . . . . . . 62

4.3 .1 Classical Theory . . . . . . . . . . . . . . . . . . 62

4.3.2 Quantum Theory . . . . . . . . . . . . . . . 65

4.4 Harmonic Operators . . . . . . . . . . . . . . . . . 73

5 Quantum Group Channels $\quad 78$

5.1 Dual Channels . . . . . . . . . . . . . . . . . . . . . . 78

5.2 Commutative and Co-Commutative Quantum Groups . . . . . . . . 80

5.2 .1 Fixed Point Algebras . . . . . . . . . . . . . . . . . 86

5.2.2 The Asymptotic Quantum Birkoff Conjecture . . . . . . . . 90

5.3 The Kac-Paljutkin Algebra . . . . . . . . . . . . . . . . . . . . . 104

$\begin{array}{lr}\text { Bibliography } & 115\end{array}$

$\begin{array}{ll}\text { Appendix } & 122\end{array}$ 


\section{Chapter 1}

\section{Introduction}

In order to obtain a concrete realization of an abstract object in mathematics, one usually represents that object in a more familiar space where the algebraic and topological properties are easier to study. For instance, in the field of operator algebras it is known that every $C^{*}$-algebra $\mathcal{A}$ may be isometrically embedded into $\mathcal{B}(H)$ for some Hilbert space $H$ via the GNS construction [73]. In many cases of interest in harmonic analysis (such as $\mathcal{A}=C_{0}(G)$ for a locally compact group $G$ ), the dual $\mathcal{A}^{*}$ has a Banach algebra structure, so it is natural to consider the dual representation

problem. However, in this setting $\mathcal{B}(H)$ is not the appropriate target algebra [26].

This led mathematicians to develop a new representation framework for certain Banach algebras where the target algebra is replaced by $\mathcal{C B}(\mathcal{B}(H))$, the algebra of completely bounded maps on $\mathcal{B}(H)$. This framework incorporates the recent theory of operator spaces as an essential tool, allowing for many new applications. One 
such application, providing the backbone of the thesis, is the completely bounded multiplier algebra of a locally compact quantum group $\mathbb{G}[41]$.

In what follows we shall explore the properties of this Banach algebra and show how its representation theory leads to a new connection between harmonic analysis and quantum information. In order to establish this connection, we begin in chapter 2 with a review of the mathematical essentials in either domain: locally compact groups, their Banach algebras, and quantum channels. We also include important preliminary notions from operator spaces and von Neumann algebras, which are essential for the present work.

Chapter 3 is devoted to a brief introduction to locally compact quantum groups in the sense of Kustermans and Vaes [51]. We present the main constructions and results concerning fundamental unitaries, nonabelian Pontrjagin duality, and quantum groups in the universal setting [48].

In chapter 4 , we study completely bounded multipliers of locally compact quantum groups and their representation theory [41]. In this vein, we also study positive definite functionals and their connection with unitary co-representations. As an application, we show that every normalized positive definite functional on a locally compact quantum group yields a quantum channel via the Junge-Neufang-Ruan representation theorem [41]. Furthermore, we introduce a systematic method of constructing the resulting channel in terms of its unitary co-representation. We finish the chapter with a description of the fixed points for this class of quantum channels, i.e., the 
quantum harmonic operators studied in [46].

The final chapter contains the main applications to quantum information theory. Focusing primarily on commutative and co-commutative quantum groups, we study several concrete examples and discuss their connections to quantum error correction. In particular, we provide new counter-examples to two recently solved conjectures concerning fixed point algebras in infinite dimensions and the asymptotic nature of bistochastic channels. We also study the relation between quantum channels arising from dual quantum groups, and see that this duality sheds new insight into the structure of some well known channels. In the final section, we study those quantum channels obtained via the smallest nontrivial quantum group, the Kac-Paljutkin algebra [42]. By explicitly computing the (left) fundamental unitary, we arrive at a concrete expression of the quantum channel for any (normalized) positive definite functional. We finish with a discussion on the structure of the fixed points of these channels by computing the idempotent states and comparing the results with the classical setting. 


\section{Chapter 2}

\section{Preliminaries}

\subsection{Topological Groups}

Definition 2.1.1. A topological group is a set $G$ that has the structure of a group and of a topological space such that the group operations $(s, t) \mapsto s t$ and $s \mapsto s^{-1}$ are continuous (where the topology on $G \times G$ is the product topology). If the topology on $G$ is Hausdorff and locally compact, then $G$ is called a locally compact group.

Arguably the most important object encountered in elementary mathematics, $\mathbb{R}$, is a locally compact group. A crucial feature of this group is that it possesses a nontrivial translation invariant measure, namely the Lebesgue measure.

Definition 2.1.2. Let $G$ be a locally compact group. A left (respectively, right) Haar measure on $G$ is a nonzero regular Borel measure $\mu$ that satisfies $\mu(s A)=\mu(A)$ (respectively, $\mu(A s)=\mu(A))$ for every Borel set $A \subseteq G$ and every $s \in G$. 
One of the crowning achievements of harmonic analysis is the following theorem, which allows us to generalize Fourier analysis to the setting of locally compact groups.

Theorem 2.1.3. [25, Theorem 2.10] Every locally compact group possesses a left and a right Haar measure which are unique up to positive multiplicative constants.

Given a left Haar measure $\mu_{G}$ on a locally compact group $G$, for each $t \in G$ the formula $\mu_{t}(A)=\mu_{G}(A t)$ defines a regular Borel measure on $G$. By translational invariance of $\mu_{G}$, we have $\mu_{t}(s A)=\mu_{G}(s A t)=\mu_{G}(A t)=\mu_{t}(A)$ for every Borel subset $A$ and every $s \in G$. Thus $\mu_{t}$ is a left Haar measure, so there exists a positive number $\Delta(t)$ such that $\mu_{t}=\Delta(t) \mu_{G}$. The function $\Delta: G \rightarrow \mathbb{R}$ defined in this way is called the modular function of $G$. It is easily seen that $\Delta$ is determined by the group and does not depend on a particular Haar measure.

Let $L^{\infty}(G)$ be the space of essentially bounded complex-valued Borel measurable functions on $G$. A bounded linear functional $m: L^{\infty}(G) \rightarrow \mathbb{C}$ is called a left invariant mean if

$$
\langle m, 1\rangle=\|m\|=1 \quad \text { and } \quad m\left(l_{s} f\right)=m(f)
$$

for all $s \in G, f \in L^{\infty}(G)$ where $l_{s} f(t)=f(s t)$. A locally compact group $G$ is amenable if there is a left invariant mean on $L^{\infty}(G)$.

Similarly to Banach space theory, there is a notion of duality in the setting of locally compact abelian groups. On the real line this duality has been studied via Fourier analysis which has produced some of the most important applications of mathematics to the natural sciences. The generalization to arbitrary locally compact 
abelian groups was done in the early 20 th century. We shall now present a brief outline, as it provides one of the main motivations for studying quantum groups.

Definition 2.1.4. Let $G$ be a locally compact abelian group and let $\mathbb{T}$ denote the group of complex numbers of modulus one. A character on $G$ is a continuous homomorphism $\chi: G \rightarrow \mathbb{T}$.

The set of characters on $G$, denoted $\hat{G}$, forms an abelian group under pointwise multiplication, and is called the dual group of $G$. Given the topology of compact convergence, the dual group becomes a locally compact abelian group such that each $s \in G$ induces a character $\Phi(s)$ on $\hat{G}$ via $\Phi(s)(\chi)=\chi(s)$. In fact, these are all the characters.

Theorem 2.1.5 (Pontrjagin Duality Theorem). [25, Theorem 4.31] Let $G$ be a locally compact abelian group. The mapping $G \ni s \mapsto \Phi(s) \in \hat{\hat{G}}$ is a homeomorphism of topological groups.

If $X$ is a locally compact Hausdorff space, we denote by $C_{0}(X)$, the space of continuous functions on $X$ vanishing at infinity. We also denote by $L^{1}(G)$, the Lebesgue space of integrable functions with respect to a fixed left Haar measure on a locally compact group $G$. When $G$ is abelian, the Fourier transform is the mapping $\mathcal{F}: L^{1}(G) \rightarrow C_{0}(\hat{G})$ given by

$$
\mathcal{F}(f)(\chi)=\int_{G} \overline{\chi(s)} f(s) d \mu_{G}(s), \quad f \in L^{1}(G), \quad \chi \in \hat{G},
$$


where $\mu_{G}$ is the Haar measure on $G$.

The corresponding duality theory for nonabelian locally compact groups took mathematicians over 40 years to complete. At this point in time it remains a beautiful, albeit technically challenging area of mathematics relying heavily on the modern theory of operator algebras. A topic to which we now turn.

\subsection{Banach Algebras}

Definition 2.2.1. A Banach algebra is an algebra $\mathcal{A}$ over the field of complex numbers equipped with a norm with respect to which it is a Banach space and which satisfies $\|a b\| \leq\|a\|\|b\|$ for all $a, b \in \mathcal{A}$. $\mathcal{A}$ is said to

- be unital if it contains a multiplicative identity, which we denote by 1 ;

- have a bounded approximate identity if there exists a bounded net $\left(e_{\alpha}\right)_{\alpha \in A}$ satisfying

$$
\lim _{\alpha} e_{\alpha} a=\lim _{\alpha} a e_{\alpha}=a \quad \text { for all } \quad a \in \mathcal{A} \text {. }
$$

Remark 2.2.2. In what follows, if $\mathcal{A}$ is a unital Banach algebra, then a unital subalgebra of $\mathcal{A}$ will mean a subalgebra $\mathcal{B} \subseteq \mathcal{A}$ containing the identity of $\mathcal{A}$. 
Definition 2.2.3. $\quad$ - An involution on an algebra $\mathcal{A}$ is a map ${ }^{*}: \mathcal{A} \rightarrow \mathcal{A}$ that satisfies

$$
(a+b)^{*}=a^{*}+b^{*},(\lambda a)^{*}=\bar{\lambda} a^{*},(a b)^{*}=b^{*} a^{*}, a^{* *}=a
$$

for all $a, b \in \mathcal{A}, \lambda \in \mathbb{C}$. An algebra equipped with an involution is called an involutive algebra.

- An involutive Banach algebra whose $*$-operation is an isometry is called a $B a$ nach *-algebra.

- An involutive Banach algebra $\mathcal{A}$ that satisfies

$$
\left\|a^{*} a\right\|=\|a\|^{2} \quad \text { for all } a \in \mathcal{A}
$$

is called a $C^{*}$-algebra.

The prototypical example of a $C^{*}$-algebra is $\mathcal{B}(H)$, the space of bounded linear operators on a Hilbert space, with the operator norm and involution given by the adjoint operation. The space $\mathcal{B}(H)$ is the dual of the Banach space of trace-class operators, denoted $\mathcal{T}(H)$, where an element $\rho \in \mathcal{B}(H)$ is called trace-class if $\operatorname{tr}|\rho|<$ $\infty$, with tr denoting the canonical trace of an operator with respect to an orthonormal basis of $H$. The duality between $\mathcal{B}(H)$ and $\mathcal{T}(H)$ is given by

$$
\langle\rho, T\rangle=\operatorname{tr}(\rho T) \quad \rho \in \mathcal{T}(H), \quad T \in \mathcal{B}(H) .
$$


If $\left(e_{\imath}\right)_{\imath \in I}$ is an orthonormal basis for $H$, we denote by $\theta_{e_{2}, e_{j}}$, the rank-one operator in $\mathcal{T}(H)$ defined by $\theta_{e_{2}, e_{j}}(\xi)=\left\langle\xi, e_{\jmath}\right\rangle e_{2}, \xi \in H$.

Given a Banach algebra $\mathcal{A}$, one defines an $\mathcal{A}$-bimodule structure on $\mathcal{A}^{*}$ given by

$$
\langle a \cdot \varphi, b\rangle=\langle\varphi, b a\rangle \quad \text { and } \quad\langle\varphi \cdot a, b\rangle=\langle\varphi, a b\rangle, \quad \varphi \in \mathcal{A}^{*}, \quad a, b \in \mathcal{A} .
$$

It is known that there exists left and right Arens products on the bidual $\mathcal{A}^{* *}[2]$, denoted $\square$ and $\diamond$, respectively, which are defined by

$$
\langle m \square n, \varphi\rangle=\langle m, n \square \varphi\rangle \quad \text { and } \quad\langle m \diamond n, \varphi\rangle=\langle n, \varphi \diamond m\rangle \quad m, n \in \mathcal{A}^{* *}, \quad \varphi \in \mathcal{A}^{*}
$$

where $n \square \varphi$ and $\varphi \diamond m$ are the elements in $\mathcal{A}^{*}$ given by

$$
\langle n \square \varphi, a\rangle=\langle n, \varphi \cdot a\rangle \quad \text { and } \quad\langle\varphi \diamond m, a\rangle=\langle m, a \cdot \varphi\rangle, \quad a \in \mathcal{A} .
$$

It follows that $\left(\mathcal{A}^{* *}, \square\right)$ and $\left(\mathcal{A}^{* *}, \diamond\right)$ are Banach algebras extending the product in $\mathcal{A}$. When the left and right Arens products on $\mathcal{A}^{* *}$ coincide, $\mathcal{A}$ is said to be Arens regular. For example, it can be shown that $C^{*}$-algebras are Arens regular [62, Theorem 2.11.3]. By construction, if one of the factors $m$ or $n$ belongs to $\mathcal{A} \subseteq \mathcal{A}^{* *}$, then the left and right products agree.

Much of abstract harmonic analysis concerns the study of Banach algebras associated to a locally compact group. Here we discuss the main examples that will be important throughout the thesis, with $G$ denoting a fixed locally compact group with 
left Haar measure $\mu_{G}$.

Example 2.2.4. The space $C_{0}(G)$ with the uniform norm, pointwise multiplication and involution given by complex conjugation, forms a commutative $C^{*}$-algebra. In fact, every commutative $C^{*}$-algebra is of the form $C_{0}(X)$ for a locally compact Hausdorff space $X\left[73\right.$, Theorem 4.4], suggesting that $C^{*}$-algebras play the role of "noncommutative topological spaces".

By the Riesz representation theorem, the Banach space dual $C_{0}(G)^{*}$ is isometrically isomorphic to $M(G)$, the space of bounded complex regular Borel measures on $G$ with total variation norm. The duality is given by

$$
\langle\mu, f\rangle=\int_{G} f(s) d \mu(s), \quad \mu \in M(G), \quad f \in C_{0}(G)
$$

Example 2.2.5. The space $M(G)$ becomes a Banach algebra when equipped with the convolution product, defined by

$$
\langle\mu * \nu, f\rangle=\int_{G} \int_{G} f(s t) d \mu(s) d \nu(t), \quad \mu, \nu \in M(G), \quad f \in C_{0}(G) .
$$

The resulting algebra is called the measure algebra of $G$, and is commutative if and only if $G$ is abelian. With the involution $\mu^{*}(A)=\overline{\mu\left(A^{-1}\right)}, A \subseteq G$ Borel, $M(G)$ becomes a Banach $*$-algebra.

Example 2.2.6. We may identify $L^{1}(G)$ with a norm closed ideal in $M(G)$ consisting of those measures absolutely continuous with respect to the Haar measure. With this 
identification, the convolution of $f$ and $g$ in $L^{1}(G)$ is the function

$$
(f * g)(s)=\int_{G} f(t) g\left(t^{-1} s\right) d \mu_{G}(t), \quad s \in G .
$$

The restricted involution on $L^{1}(G)$ takes the form $f^{*}(s)=\Delta\left(s^{-1}\right) \overline{f\left(s^{-1}\right)}, s \in G$. Under the convolution product and the above involution, $L^{1}(G)$ also becomes a Banach *-algebra, called the group algebra of $G$.

Throughout mathematics, especially quantum mechanics, the notion of positivity plays a crucial role. In the setting of $C^{*}$-algebras, it provides a deep connection with representation theory whose manifestation at the level of groups comprises some of the most beautiful results in harmonic analysis (cf. $§ 4.3 .1)$.

Definition 2.2.7. An element $a$ in a Banach $*$-algebra $\mathcal{A}$ said to be positive, denoted $a \geq 0$, if $a=b^{*} b$ for some $b \in \mathcal{A}$. We denote the set of positive operators by $\mathcal{A}^{+}$.

A linear map $\varphi: \mathcal{A} \rightarrow \mathcal{B}$ between Banach $*$-algebras is said to be positive if $\varphi\left(\mathcal{A}^{+}\right) \subseteq \mathcal{B}^{+}$. In the special case where $\mathcal{B}=\mathbb{C}$, we say that $\varphi$ is a positive linear functional. The collection of positive linear functionals of norm one, denoted $\mathcal{S}(\mathcal{A})$, is called the state space of $\mathcal{A}$. When $\mathcal{A}$ is a $C^{*}$-algebra, $\mathcal{S}(\mathcal{A})$ forms a convex subset of the closed unit ball of $\mathcal{A}^{*}[62$, Proposition 2.3.7]. Its extreme points are called pure states. In this case, we say that a state $\varphi \in \mathcal{S}(\mathcal{A})$ is tracial if $\varphi(a b)=\varphi(b a)$ for all $a, b \in \mathcal{A}$. 
Remark 2.2.8. If $\mathcal{A}$ is a unital Banach $*$-algebra, then a positive linear functional $\varphi$ is a state if and only if $\varphi(1)=1\left[73\right.$, Lemma 9.9]. In this case, $\mathcal{S}(\mathcal{A})$ is a weak ${ }^{*}$ closed subset of the closed unit ball of $\mathcal{A}^{*}$, and is therefore compact by Alaoglu's theorem.

Definition 2.2.9. A $*$-representation of a Banach $*$-algebra $\mathcal{A}$ is a $*$-homomorphism $\pi: \mathcal{A} \rightarrow \mathcal{B}\left(H_{\pi}\right)$, where $H_{\pi}$ is a Hilbert space. We say that $\pi$ is:

- spatially nondegenerate, or s.nd. for short, if $\pi(\mathcal{A}) H_{\pi}=\{\pi(a) \xi: a \in \mathcal{A}, \quad \xi \in$ $\left.H_{\pi}\right\}$ is dense in $H_{\pi}$;

- cyclic if there is a vector $\xi \in H_{\pi}$ such that $\pi(\mathcal{A}) \xi$ is dense in $H_{\pi}$;

- irreducible if there are no nontrivial proper invariant subspaces of $H_{\pi}$ under $\pi(\mathcal{A})$

- faithful if $\pi$ is injective.

Two $*$-representations $\pi_{1}$ and $\pi_{2}$ are said to be unitarily equivalent if there exists a unitary $U: H_{\pi_{1}} \rightarrow H_{\pi_{2}}$ such that $\pi_{2}(a)=U \pi_{1}(a) U^{*}$ for all $a \in \mathcal{A}$. It follows that any *-representation of a $C^{*}$-algebra is bounded [62, Proposition 2.3.17].

Example 2.2.10. For $f \in L^{1}(G)$, define

$$
\|f\|_{*}=\sup \left\{\|\pi(f)\| \mid \pi: L^{1}(G) \rightarrow \mathcal{B}\left(H_{\pi}\right) \text { is a s.nd. *-representation }\right\} .
$$

It is easily seen that $\|\cdot\|_{*}$ is a norm on $L^{1}(G)$ satisfying $\|\cdot\|_{*} \leq\|\cdot\|_{1}$, whose completion becomes a $C^{*}$-algebra, denoted $C^{*}(G)$, called the (full) group $C^{*}$-algebra of $G$. In 
fact, $C^{*}(G)$ is the enveloping $C^{*}$-algebra of the Banach *-algebra $L^{1}(G)$.

Given a $*$-representation $\pi: \mathcal{A} \rightarrow \mathcal{B}(H)$ of a Banach $*$-algebra and a vector $\xi \in H_{\pi}$, the function $\varphi(x)=\langle\pi(x) \xi, \xi\rangle, x \in \mathcal{A}$, defines a positive linear functional on $\mathcal{A}$. The converse of this result is known as the GNS construction.

Theorem 2.2.11. [73, Theorem 9.14] If $\varphi$ us a positive linear functional on a Banach

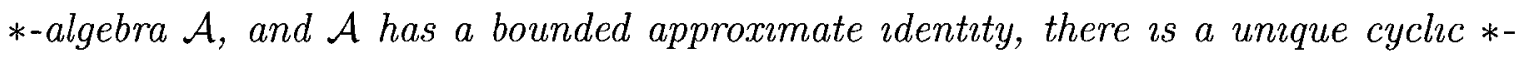

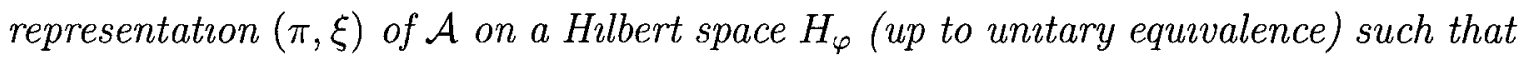
$\varphi(x)=\langle\pi(x) \xi, \xi\rangle$ for all $x \in \mathcal{A}$. Moreover, if $\varphi \in \mathcal{S}(\mathcal{A})$, then $\varphi$ us pure if and only if $\pi$ us ırreducıble.

As every $C^{*}$-algebra has a bounded approximate identity $[62, \S 2.4]$, one obtains:

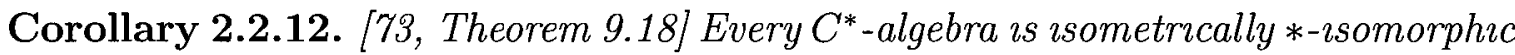
to a $C^{*}$-subalgebra of $\mathcal{B}(H)$ for some Hilbert space $H$.

Given two $C^{*}$-algebras $\mathcal{A}$ and $\mathcal{B}$, acting on Hilbert spaces $H$ and $K$, respectively, the algebraic tensor product $\mathcal{A} \otimes \mathcal{B}$ acts naturally on the H1lbert space tensor product $H \otimes_{2} K$, and its norm closure inside $\mathcal{B}(H) \otimes \mathcal{B}(K)$ is a $C^{*}$-algebra called the minımal $C^{*}$-tensor product of $\mathcal{A}$ and $\mathcal{B}$, denoted $\mathcal{A} \otimes_{\text {min }} \mathcal{B}$. It can be shown that this definition is independent of the Hilbert space representation [62, §3.2]. Furthermore, denoting the identity map by $\iota$, if $\varphi \in \mathcal{A}^{*}$, then the right slice map $\varphi \otimes \iota: \mathcal{A} \otimes \mathcal{B} \rightarrow \mathcal{B}$ defined on elementary tensors by

$$
(\varphi \otimes \iota)(a \otimes b)=\varphi(a) b, \quad a \in \mathcal{A}, \quad b \in \mathcal{B},
$$


extends uniquely to a bounded linear map $\varphi \otimes \iota: \mathcal{A} \otimes_{\operatorname{m\imath n}} \mathcal{B} \rightarrow \mathcal{B}[62, \S 3.2]$.

\subsection{Operator Spaces}

In 1925, our view of the quantum world drastically changed with the advent of Heisenberg's matrix mechanics. He showed that we may accurately describe quantum phenomena by interpreting time dependent variables as infinite matrices rather than functions. This "quantization" of functions motivated mathematicians to quantize other areas of mathematics. One such quantization, mainly developed by EffrosRuan and Blecher-Paulsen is the theory of operator spaces, which may be viewed as a quantization of Banach space theory.

During the late 20th century, mainly due to results of Effros, Haagerup and Ruan, it became apparent that the category of operator spaces is the natural context in which to view the various Banach algebras associated to a locally compact group. This created an entirely new facet of abstract harmonic analysis, which in particular generated the representation theoretical framework we shall study in this thesis. In order to establish this framework, we review the main definitions and examples from operator space theory in this section.

If $V$ is a linear space, for $m, n \in \mathbb{N}$ we let $M_{m, n}(V)$ denote the space of $m \times n$ matrices with entries in $V$. If $m=n$, we simply denote $M_{m, n}(V)$ by $M_{n}(V)$.

A matrix norm on a linear space $V$ is a sequence of norms $\left(\|\cdot\|_{n}\right)_{n \in \mathbb{N}}$ where $\|\cdot\|_{n}$ is defined on $M_{n}(V)$ for every $n \in \mathbb{N}$. An abstract operator space is a linear space $V$ 
together with a matrix norm such that each $\|\cdot\|_{n}$ is complete and satisfies

- R1 $\|v \oplus w\|_{m+n}=\max \left\{\|v\|_{m},\|w\|_{n}\right\}$,

- R2 $\|\alpha v \beta\|_{n} \leq\|\alpha\|\|v\|_{m}\|\beta\|$,

for all $m, n \in \mathbb{N}, v \in M_{m}(V), w \in M_{n}(V), \alpha \in M_{n, m}(\mathbb{C}), \beta \in M_{m, n}(\mathbb{C})$. Such a matrix norm is called an operator space matrix norm.

Given abstract operator spaces $V, W$ and a linear mapping $\Phi: V \rightarrow W$, for each $n \in \mathbb{N}$ there is a corresponding linear map $\Phi_{n}: M_{n}(V) \rightarrow M_{n}(W)$ given by

$$
\Phi_{n}(v)=\left[\Phi\left(v_{\imath \jmath}\right)\right]
$$

for all $v=\left[v_{\imath \jmath}\right] \in M_{n}(V)$, called the $n^{\text {th }}$ amplification of $\Phi$.

Definition 2.3.1. Let $V$ and $W$ be abstract operator spaces. A linear map $\Phi: V \rightarrow$ $W$ is called:

- completely bounded if $\|\Phi\|_{c b}:=\sup \left\{\left\|\Phi_{n}\right\|: n \in \mathbb{N}\right\}<\infty$;

- completely contractive if $\|\Phi\|_{c b} \leq 1$;

- a complete isometry if $\Phi_{n}$ is an isometry for all $n \in \mathbb{N}$;

- a complete quotient map if $\Phi_{n}$ is a quotient map for all $n \in \mathbb{N}$.

If, in addition, $V$ and $W$ are self-adjoint unital subspaces of $\mathcal{B}(H)$ and $\mathcal{B}(K)$ respectively, then we say that $\Phi$ is completely positive if $\Phi_{n}$ is positive for all $n \in \mathbb{N}$. In 
this case, it follows that $\Phi$ is automatically completely bounded with $\|\Phi\|_{c b}=\|\Phi\|=$ $\|\Phi(1)\|[61$, Proposition 3.6].

The prototypical example of an abstract operator space is a closed subspace $V$ of $\mathcal{B}(H)$ for some Hilbert space $H$. The matrix norms are defined by the identification $M_{n}(V) \subseteq M_{n}(\mathcal{B}(H)) \cong \mathcal{B}\left(H^{n}\right)$, where $H^{n}$ is the $n$-fold Hilbert space direct sum and $n \in \mathbb{N}$. These are known as concrete operator spaces, and in fact, there is a bijective correspondence.

Theorem 2.3.2 (Ruan's Theorem). [65] Every abstract operator space is completely isometrically isomorphic to a concrete operator space.

In what follows, we will therefore omit the adjectives abstract and concrete and simply use the term operator space.

There are many interesting tensor product structures in operator space theory. One that will be important for us is the operator space projective tensor product, which is defined as follows. If $V$ and $W$ are operator spaces, given $u \in M_{n}(V \otimes W)$, we define

$$
\|u\|_{\wedge}=\inf \{\|\alpha\|\|v\|\|w\|\|\beta\|: u=\alpha(v \otimes w) \beta\}
$$

where the infimum is taken over all possible decompositions with $v \in M_{p}(V), w \in$ $M_{q}(V), \alpha \in M_{n, p q}(\mathbb{C}), \beta \in M_{p q, n}(\mathbb{C})$ and $p, q \in \mathbb{N}$. One may verify that this is indeed an operator space matrix norm. The completion of the algebraic tensor product $V \otimes W$ under this matrix norm becomes an operator space, called the operator space projective tensor product, denoted by $V \widehat{\otimes} W$. 
Given operator spaces $V$ and $W$, we denote the set of completely bounded mappings from $V$ to $W$ by $\mathcal{C B}(V, W)$. Note that each $\Phi \in M_{n}(\mathcal{C B}(V, W))$ may be identified with an element of $\mathcal{C B}\left(V, M_{n}(W)\right)$, for $n \in \mathbb{N}$. By defining

$$
\|\Phi\|_{M_{n}(\mathcal{C B}(V, W))}:=\|\Phi\|_{\mathcal{C B}\left(V, M_{n}(W)\right)},
$$

it follows that $\|\cdot\|_{M_{n}(\mathcal{C B}(V, W))}$ is an operator space matrix norm, turning $\mathcal{C B}(V, W)$ into an operator space in its own right. Since every bounded linear functional $\varphi: V \rightarrow \mathbb{C}$ is completely bounded [61, Proposition 3.8], $V^{*}=\mathcal{C B}(V, \mathbb{C})$. Thus, the dual of an operator space carries a canonical operator space structure. In particular, norm closed subspaces of $V^{*}$ are operator spaces, a fact we shall consider without comment in the remainder of the thesis.

As is customary, if $V=W$ we simply write $\mathcal{C B}(V)$ for $\mathcal{C B}(V, V)$. Under composition, the space $\mathcal{C B}(V)$ becomes a completely contractive Banach algebra, meaning that multiplication in $\mathcal{C B}(V)$, seen as a map $\mathcal{C B}(V) \widehat{\otimes} \mathcal{C B}(V) \rightarrow \mathcal{C B}(V)$ is a completely contractive operation.

\section{4 von Neumann Algebras}

For a locally compact group $G$, the algebra $L^{\infty}(G)$ belongs to a special class of $C^{*}$ algebras, known as von Neumann algebras. To discuss elements of the theory of von Neumann algebras, we must first introduce further interesting topologies on $\mathcal{B}(H)$. 
1. The strong operator topology (SOT) is the topology induced by the seminorms $\mathcal{B}(H) \ni T \mapsto\|T u\|, u \in H$. A net $\left(T_{\alpha}\right)_{\alpha \in A}$ converges to $T$ strongly if and only if $\left\|T_{\alpha} u-T u\right\| \rightarrow 0$ for every $u \in H$.

2. The weak operator topology (WOT) is the topology induced by the seminorms $\mathcal{B}(H) \ni T \mapsto|\langle T u, v\rangle|, u, v \in H$. A net $\left(T_{\alpha}\right)_{\alpha \in A}$ converges to $T$ weakly if and only if $\left\langle T_{\alpha} u, v\right\rangle \rightarrow\langle T u, v\rangle$ for every $u, v \in H$.

3. The weak* topology is the topology defined by the seminorms $\mathcal{B}(H) \ni T \mapsto$ $|\operatorname{tr}(T \rho)|, \rho \in \mathcal{T}(H)$. A net $\left(T_{\alpha}\right)_{\alpha \in A}$ converges to $T$ weak* if and only if $\operatorname{tr}\left(T_{\alpha} \rho\right) \rightarrow$ $\operatorname{tr}(T \rho)$ for every $\rho \in \mathcal{T}(H)$.

Definition 2.4.1. A von Neumann algebra $M$ acting on a Hilbert space $H$ is a unital $C^{*}$-subalgebra of $\mathcal{B}(H)$ that is closed under the strong operator topology.

For a subset $\mathcal{S}$ of $B(H)$, the commutant of $\mathcal{S}$, denoted $\mathcal{S}^{\prime}$, is the set of operators in $\mathcal{B}(H)$ that commute with every element of $\mathcal{S}$, that is

$$
\mathcal{S}^{\prime}=\{T \in \mathcal{B}(H): T S=S T \text { for all } S \in \mathcal{S}\}
$$

The double commutant or bicommutant of $\mathcal{S}$ is defined by $\mathcal{S}^{\prime \prime}=\left\{\mathcal{S}^{\prime}\right\}^{\prime}$. One of the many beautiful results in the theory of operator algebras relates this purely algebraic concept with the above topologies in the setting of $C^{*}$-algebras. 
Theorem 2.4.2 (von Neumann's Double Commutant Theorem). [15, Theorem 12.3] If $M$ is a self-adjoint unital subalgebra of $\mathcal{B}(H)$, then the weak, weak ${ }^{*}$ and strong operator closures of $M$ coincide with $M^{\prime \prime}$.

Another distinguishing feature of a von Neumann algebra $M \subseteq \mathcal{B}(H)$ is that it is (isometrically isomorphic to) the dual of a unique Banach space $M_{*}$, called the predual of $M$, which consists of all weak* continuous linear functionals on $M$ [66].

Definition 2.4.3. If $M$ and $N$ a von Neumann algebras, then a linear (or anti-linear) map $\Phi: M \rightarrow N$ is said to be normal if it is weak*-weak* continuous.

If $M \subseteq \mathcal{B}(H)$, observe that for $\xi, \eta \in H$, the linear functional $\omega_{\xi, \eta}: M \rightarrow \mathbb{C}$ defined by $\left\langle\omega_{\xi, \eta}, x\right\rangle=\langle x \xi, \eta\rangle, x \in M$, is normal. For simplicity, we denote $\omega_{\xi, \xi}$ by $\omega_{\xi}$. Note that $\omega_{\xi}=\theta_{\xi, \xi}$ under the notation of $\S 2.2$ and that the adjoint operation in $M$ is also normal.

Throughout the thesis, when $H$ is a Hilbert space, the algebra of normal completely bounded maps on $\mathcal{B}(H)$ will be denoted $\mathcal{C B}^{\sigma}(\mathcal{B}(H)$ ). If $\mathcal{A}$ is subspace of $\mathcal{B}(H)$, and $\mathcal{B}$ is a subalgebra of $\mathcal{B}(H)$, we denote by $\mathcal{C B}_{\mathcal{B}}^{\sigma, \mathcal{A}}(\mathcal{B}(H))$, the algebra of normal completely bounded $\mathcal{B}$-bimodule maps on $\mathcal{B}(H)$ that leave $\mathcal{A}$ invariant, that is, those completely bounded maps $\Phi$ on $\mathcal{B}(H)$ that satisfy

$$
\begin{gathered}
\Phi(b T c)=b \Phi(T) c, \quad T \in \mathcal{B}(H), \quad b, c \in \mathcal{B} \quad \text { and } \\
\Phi(a) \in \mathcal{A}, \quad a \in \mathcal{A} .
\end{gathered}
$$


Example 2.4.4. Let $(X, \Sigma, \mu)$ be a $\sigma$-finite measure space. Under pointwise multiplication, the space of (complex-valued) essentially bounded $\mu$-measurable functions $L^{\infty}(X, \mu)$ becomes a commutative von Neumann algebra when viewed as the algebra of multiplication operators on $L^{2}(X, \mu)$. In fact, every commutative von Neumann algebra arises in this fashion for a suitable measure space $(X, \Sigma, \mu)[44, \S 9.4]$, suggesting that von Neumann algebras play the role of "noncommutative measure spaces".

If $G$ is a locally compact group, $L^{\infty}(G)$ is a von Neumann algebra with predual $L^{1}(G)[25, \S 2.3]$. For the remainder of the thesis, elements of $L^{\infty}(G)$ will be viewed as functions and/or multiplication operators when convenient. We will denote functions by $f$ and their corresponding multiplication operators by $M_{f}$.

Many important von Neumann algebras in abstract harmonic analysis arise from representations of a locally compact group $G$, the two main examples being the left and right regular representations, given, respectively, by $\lambda: G \rightarrow \mathcal{B}\left(L^{2}(G)\right)$ and $\rho: G \rightarrow \mathcal{B}\left(L^{2}(G)\right)$, where

$$
\lambda(s) \xi(t)=\xi\left(s^{-1} t\right) \quad \text { and } \quad \rho(s) \xi(t)=\Delta(s)^{1 / 2} \xi(t s)
$$

for $\xi \in L^{2}(G), s, t \in G$. It follows that

$$
\mathcal{L}(G):=\overline{\operatorname{span}\{\lambda(s): s \in G\}}^{S O T} \quad \text { and } \quad \mathcal{R}(G):=\overline{\operatorname{span}\{\rho(s): s \in G\}}^{S O T}
$$


are von Neumann algebras in $\mathcal{B}\left(L^{2}(G)\right)$, called the left and right group von Neumann algebras, respectively. Furthermore, they satisfy the following commutation relations:

$$
\mathcal{L}(G)^{\prime}=\mathcal{R}(G) \quad \text { and } \quad \mathcal{R}(G)^{\prime}=\mathcal{L}(G)
$$

Remark 2.4.5. By lifting the left regular representation to $\lambda: L^{1}(G) \rightarrow \mathcal{B}\left(L^{2}(G)\right)$ via:

$$
\langle\lambda(f), \rho\rangle=\int_{G} f(s)\langle\lambda(s), \rho\rangle d \mu_{G}(s), \quad f \in L^{1}(G), \quad \rho \in \mathcal{T}\left(L^{2}(G)\right)
$$

it follows that

$$
\mathcal{L}(G)={\overline{\operatorname{span}\left\{\lambda(f): f \in L^{1}(G)\right\}}}^{S O T}
$$

If instead we take the norm closure in equation (2.2), then we obtain a $C^{*}$-algebra, denoted $C_{\lambda}^{*}(G)$, known as the left reduced $C^{*}$-algebra of $G$. Similarly, we have the corresponding result for the right regular representation.

The set of coefficient functions of the left regular representation,

$$
A(G)=\left\{f: G \rightarrow \mathbb{C}: f(s)=\langle\lambda(s) \xi, \eta\rangle, \quad \xi, \eta \in L^{2}(G), \quad s \in G\right\}
$$

is called the Fourier algebra of $G$. It was shown by Eymard that, endowed with the norm $\|f\|=\inf \{\|\xi\|\|\eta\|: f(\cdot)=\langle\lambda(\cdot) \xi, \eta\rangle\}, A(G)$ is a Banach algebra under pointwise multiplication [23, Proposition 3.4]. Furthermore, it is the predual of the 
left group von Neumann algebra $\mathcal{L}(G)$, where the duality is given by

$$
\langle\psi, \lambda(s)\rangle=\psi(s), \quad \psi \in A(G), \quad s \in G
$$

When $G$ is abelian with dual group $\hat{G}$, the algebra $A(\hat{G})$ is simply the image of the Fourier transform $\mathcal{F}: L^{1}(G) \rightarrow C_{0}(\hat{G})$. The dual mapping $\mathcal{F}^{*}: \mathcal{L}(\hat{G}) \rightarrow L^{\infty}(G)$ therefore provides a von Neumann algebraic isomorphism. In this sense, $L^{\infty}(G)$ and $\mathcal{L}(G)$ are "dual" to one another. In fact, it was this correspondence that set the stage for the duality theory of arbitrary locally compact groups, and hence the theory of locally compact quantum groups, which utilizes the theory of noncommutative integration.

Definition 2.4.6. A weight on a von Neumann algebra $M$ is a function $\varphi: M^{+} \rightarrow$ $[0, \infty]$ satisfying:

1. $\varphi(x+y)=\varphi(x)+\varphi(y) \quad$ for all $\quad x, y \in M^{+}$

2. $\varphi(r x)=r \varphi(x) \quad$ for all $\quad r \in \mathbb{R}^{+}, \quad x \in M^{+}$.

The fact that weights may be viewed as noncommutative integrals is justified by the following canonical example.

Example 2.4.7. If $(X, \Sigma, \mu)$ is a $\sigma$-finite measure space, then the function

$$
\varphi(f)=\int_{X} f(s) d \mu(s), \quad 0 \leq f \in L^{\infty}(X, \mu)
$$


is a weight on the commutative von Neumann algebra $L^{\infty}(X, \mu)$.

Given a weight $\varphi$ on a von Neumann algebra $M$, we adopt the following standard notations:

- $\mathcal{M}_{\varphi}^{+}=\left\{x \in M^{+}: \varphi(x)<\infty\right\}$

- $\mathcal{N}_{\varphi}=\left\{x \in M: \varphi\left(x^{*} x\right)<\infty\right\}$

- $\mathcal{M}_{\varphi}=\operatorname{span}\left\{x^{*} y: x, y \in \mathcal{N}_{\varphi}\right\}$.

It follows that $\mathcal{N}_{\varphi}$ is a left ideal in $M$, and that $\mathcal{M}_{\varphi}=\operatorname{span} \mathcal{M}_{\varphi}^{+}$is a ${ }^{*}$-subalgebra of $M$ [74, Lemma 1.2]. We may therefore extend $\varphi$ to a positive linear form on $\mathcal{M}_{\varphi}$, which will also be denoted by $\varphi$.

In the classical setting of Example 2.4.7, $\mathcal{M}_{\varphi}$ and $\mathcal{N}_{\varphi}$ correspond to $L^{\infty}(X, \mu) \cap$ $L^{1}(X, \mu)$ and $L^{\infty}(X, \mu) \cap L^{2}(X, \mu)$, respectively. In general, we say that the weight $\varphi$ is:

- semi-finite if $\mathcal{M}_{\varphi}$ is weakly dense in $M$;

- faithful if $\varphi(x)=0$ implies $x=0$ for all $x \in M^{+}$;

- normal if $\varphi\left(\sup x_{\alpha}\right)=\sup \varphi\left(x_{\alpha}\right)$ for every bounded increasing net $\left(x_{\alpha}\right)_{\alpha \in A}$ in $M^{+}$

If $\varphi$ is a normal semi-finite faithful (n.s.f.) weight on a von Neumann algebra $M$, we may define an inner product on $\mathcal{N}_{\varphi}$ by

$$
\langle x, y\rangle=\varphi\left(y^{*} x\right), \quad x, y \in \mathcal{N}_{\varphi} .
$$


Denoting by $H_{\varphi}$ the Hilbert space completion of $\mathcal{N}_{\varphi}$, we obtain the canonical embed$\operatorname{ding} \Lambda_{\varphi}: \mathcal{N}_{\varphi} \rightarrow H_{\varphi}$ and a $*$-representation $\pi_{\varphi}: M \rightarrow B\left(H_{\varphi}\right)$ of $M$ given by

$$
\pi_{\varphi}(x)\left(\Lambda_{\varphi}(a)\right)=\Lambda_{\varphi}(x a), \quad x \in M, \quad a \in \mathcal{N}_{\varphi}
$$

The triple $\left(H_{\varphi}, \pi_{\varphi}, \Lambda_{\varphi}\right)$ is called the GNS-construction for $\varphi$.

In the case of Example 2.4.7, the Hilbert space $H_{\varphi}$ is nothing but $L^{2}(X, \mu)$, and the *-representation $\pi_{\varphi}: L^{\infty}(X, \mu) \rightarrow \mathcal{B}\left(L^{2}(X, \mu)\right)$ is the realization of functions as multiplication operators.

Alongside noncommutative integration, another reoccurring object in quantum group theory is the tensor product of von Neumann algebras. If $M$ and $N$ are von Neumann algebras acting on Hilbert spaces $H$ and $K$, respectively, then the algebraic tensor product $M \otimes N$ acts naturally on $H \otimes K$, and its weak* closure is a von Neumann algebra, called the von Neumann algebraic tensor product of $M$ and $N$, denoted $M \bar{\otimes} N$. It can be shown that this definition is independent of the choice of Hilbert spaces $[73, \S 4.5]$. At the level of preduals, there is a fundamental relationship with the operator space projective tensor product [20]:

$$
(M \bar{\otimes} N)_{*} \cong M_{*} \widehat{\otimes} N_{*},
$$

completely isometrically. 
If $M_{\imath}$ and $N_{\imath}$ are von Neumann algebras for $i=1,2$ and $\Phi: M_{1} \rightarrow N_{1}$ and $\Psi: M_{2} \rightarrow N_{2}$ are normal completely bounded maps, then the map $\Phi \otimes \Psi: M_{1} \otimes M_{2} \rightarrow$ $N_{1} \otimes N_{2}$ extends to a normal completely bounded map from $M_{1} \bar{\otimes} M_{2}$ to $N_{1} \bar{\otimes} N_{2}$.

Denoting the identity map by $\iota$, if $M$ and $N$ are von Neumann algebras and $\varphi \in M_{*}$, then the right slice map $\varphi \otimes \iota: M \otimes N \rightarrow N$ defined on elementary tensors by

$$
(\varphi \otimes \iota)(x \otimes y)=\varphi(x) y, \quad x \in M, y \in N
$$

extends to a completely bounded normal map from $M \bar{\otimes} N$ to $N[75, \S 1]$. Left slice maps are defined similarly. As we shall see below, these slice maps play an important role in the representation theory of locally compact quantum groups.

\subsection{Quantum Information Theory}

In the early 1980 's, Feynman put forth the idea that in order to efficiently simulate a quantum mechanical system, one would need a computer based on the principles of quantum theory, i.e., a quantum computer $[60, \S 1.1]$. Since then, much research has been done in this direction, connecting foundational ideas in modern physics, mathematics, computer science, and information theory. The resulting field, known as quantum information science, is a growing interdisciplinary science making breakthroughs in each respective subfield. In particular, the notion of a quantum computer is becoming more of a reality, with hopes and expectations to change the face of technology. This section is devoted to a brief overview of some of the fundamental concepts 
in quantum information that will be important for the thesis.

Definition 2.5.1. A quantum state on $H$ is a positive trace-class operator $\rho \in \mathcal{T}(H)$ of trace 1 . The Hilbert space $H$ is called the representation space of the quantum system, and we denote the set of quantum states on $H$ by $\mathcal{D}(H)$.

Under the identification $\mathcal{T}(H)=\mathcal{B}(H)_{*}$, the set $\mathcal{D}(H)$ is weak* closed and convex in the state space of the unital $C^{*}$-algebra $\mathcal{B}(H)$ (see Remark 2.2.8). As such, any $\rho \in$ $\mathcal{D}(H)$ may be approximated in the weak* topology by a net of convex combinations of extreme points. We call a state pure if it is extreme in $\mathcal{D}(H)$, otherwise it is said to be mixed. If $\operatorname{dim} H=n$, quantum states may be viewed as "noncommutative probability distributions". For example, the noncommutative uniform distribution is $\frac{1}{n} I_{n}$, where $I_{n}$ is the $n \times n$ identity matrix, and is called the maximally mixed state.

In classical information theory, a bit describes the state of a two-level system by assigning 0 or 1 . In quantum information theory, a "quantum bit", or qubit for short, describes the state of a two-dimensional quantum system, and is the quantum analogue of a classical bit of information. The striking difference in the quantum setting is that a qubit can be in both the " 0 " and the " 1 " state at the same time. For example, consider a hydrogen atom consisting of one proton with one orbiting electron. The spin space of the electron is two-dimensional, spanned by the states corresponding to "spin-down" (zero) and "spin-up" (one). In general, we do not know the direction of the spin until it is measured, so we describe the spin state of the electron by a probabilistic mixture of "spin-down" and "spin-up". Formally, let 
$\left\{e_{0}, e_{1}\right\}$ be the standard orthonormal basis of $\mathbb{C}^{2}$. Then the pure states $\theta_{e_{0}, e_{0}}$ and $\theta_{e_{1}, e_{1}}$ correspond to spin-down and spin-up, respectively, while in general the spin will be described by the mixed state $\rho=\alpha \theta_{e_{0}, e_{0}}+\beta \theta_{e_{1}, e_{1}}$ with $\alpha, \beta \geq 0, \alpha+\beta=1$. The coefficients $\alpha$ and $\beta$ are precisely the probabilities that a spin measurement will yield down or up, respectively.

Another important requirement in quantum information theory is an accurate mathematical model of state dynamics. In general, quantum systems are "open" in that they constantly interact with their surrounding environment. For a realistic description one then considers a system space of the form $E \otimes H$, where $E$ is the environment Hilbert space. Since $E$ may be arbitrarily large, an appropriate dynamical map must preserve positivity at all levels, i.e., be completely positive.

Completely positive maps may be viewed as a generalization of positive linear functionals of $C^{*}$-algebras [20], and as such, they have a corresponding GNS construction known as the Stinespring factorization theorem [69]. In finite dimensions this theorem implies that a linear map $\Phi: \mathcal{B}(H) \rightarrow \mathcal{B}(H)$ is completely positive if and only if there exists a set of operators $\left(a_{\imath}\right)_{i=1}^{n}$ in $\mathcal{B}(H)$ such that

$$
\Phi(x)=\sum_{\imath=1}^{n} a_{\imath} x a_{\imath}^{*}, \quad x \in \mathcal{B}(H)
$$

One can show that $\Phi$ is trace preserving if and only if $\sum_{\imath=1}^{n} a_{\imath}^{*} a_{\imath}=1$.

In the Schrödinger picture of quantum mechanics, a quantum channel is a completely positive trace preserving linear map $\Phi: \mathcal{T}(H) \rightarrow \mathcal{T}(H)$ which describes the 
evolution of quantum states. Taking the adjoint, we obtain a normal, unital completely positive map $\Phi^{*} \in \mathcal{C B}^{\sigma}(\mathcal{B}(H))$ which, in the Heisenberg picture, describes the evolution of observables such as energy and momentum. Both pictures are known to be equivalent $[70,80]$, so we shall take the Heisenberg approach.

Definition 2.5.2. A quantum channel is a normal, unital completely positive linear map $\Phi: \mathcal{B}(H) \rightarrow \mathcal{B}(H)$, where $H$ is a Hilbert space.

One advantage of this approach is the following representation theorem [28] (see also [8, Theorem 4.2]), which states that $\Phi \in \mathcal{C B}^{\sigma}(\mathcal{B}(H))$ is completely positive if and only if there is a net $\left(a_{\imath}\right)_{\imath \in I}$ in $\mathcal{B}(H)$ such that

$$
\Phi(x)=w^{*}-\sum_{\imath \in I} a_{\imath} x a_{\imath}^{*}, \quad x \in \mathcal{B}(H)
$$

and $\|\Phi\|_{c b}=\left\|w *-\sum_{\imath \in I} a_{\imath} a_{\imath}^{*}\right\|$, where $w^{*}-$ denotes the weak* limit in $\mathcal{B}(H)$. The operators $\left(a_{\imath}\right)_{\imath \in I}$ are called the Kraus operators of $\Phi$, and we say that $\Phi$ is:

- trace preserving if $\left.\Phi\right|_{\mathcal{T}(H)}: \mathcal{T}(H) \rightarrow \mathcal{T}(H)$ and $\operatorname{tr}(\Phi(\rho))=\operatorname{tr}(\rho)$ for all $\rho \in$ $\mathcal{T}(H)$

- bistochastic if it is unital and trace preserving.

In what follows, we denote the set of completely positive maps in $\mathcal{C B}^{\sigma}(\mathcal{B}(H))$ by $\mathcal{C} \mathcal{P}^{\sigma}(\mathcal{B}(H))$ 
Proposition 2.5.3. Let $H$ be a Hilbert space, and let $\Phi \in \mathcal{C P}^{\sigma}(\mathcal{B}(H))$ with Kraus operators $\left(a_{\imath}\right)_{\imath \in I}$. If $w^{*}-\sum_{\imath \in I} a_{\imath}^{*} a_{\imath}=1$, then $\Phi$ is trace preserving.

Proof. Since $w^{*}-\sum_{\imath \in I} a_{\imath}^{*} a_{\imath}=1$, the map $\tilde{\Phi}: \mathcal{B}(H) \rightarrow \mathcal{B}(H)$ defined by

$$
\tilde{\Phi}(x)=w^{*}-\sum_{\imath \in I} a_{\imath}^{*} x a_{\imath}, \quad x \in \mathcal{B}(H)
$$

is unital and satisfies $\|\tilde{\Phi}\|_{c b}=\left\|w^{*}-\sum_{\imath \in I} a_{\imath}^{*} a_{\imath}\right\|=1$. Hence, $\tilde{\Phi} \in \mathcal{C} \mathcal{P}^{\sigma}(\mathcal{B}(H))$ by [28]. Letting $\left(e_{\jmath}\right)_{\jmath \in J}$ be an orthonormal basis for $H$, we have $w^{*}-\sum_{\jmath \in J} \theta_{e_{\jmath}, e_{\jmath}}=1$, and so for $\rho \in \mathcal{T}(H)$,

$$
\begin{aligned}
\operatorname{tr}(\Phi(\rho)) & =\sum_{\jmath \in J}\left\langle\Phi(\rho), \theta_{e_{\jmath}, e_{\jmath}}\right\rangle=\sum_{\jmath \in J} \sum_{\imath \in I}\left\langle a_{\imath} \rho a_{\imath}^{*}, \theta_{e_{\jmath}, e_{\jmath}}\right\rangle \\
& =\sum_{\jmath \in J} \sum_{\imath \in I}\left\langle\rho, a_{\imath}^{*} \theta_{e_{\jmath}, e_{\jmath}} a_{\imath}\right\rangle=\sum_{\jmath \in J}\left\langle\rho, \tilde{\Phi}\left(\theta_{e_{\jmath}, e_{\jmath}}\right)\right\rangle \\
& =\langle\rho, \tilde{\Phi}(1)\rangle=\langle\rho, 1\rangle=\operatorname{tr}(\rho) .
\end{aligned}
$$

An important class of quantum channels is given by convex combinations of unitary conjugations.

Definition 2.5.4. A quantum channel $\Phi: \mathcal{B}(H) \rightarrow \mathcal{B}(H)$ is random unitary if there exists a probability space $(X, \Sigma, \mu)$ and a measurable map $U: X \rightarrow \mathcal{U}(H)$ such that

$$
\Phi(a)=\int_{X} U(x) a U(x)^{*} d \mu(x), \quad a \in \mathcal{B}(H),
$$


where $\mathcal{U}(H)$ is the group of unitary operators on $H$ and measurability is with respect to the SOT on $\mathcal{U}(H)$.

Example 2.5.5. Let $X=\{0,1\}, \Sigma=\mathcal{P}(X)$ and $0 \leq p \leq 1$. Then defining $U(0)=I_{2}$ and $U(1)=\left(\begin{array}{ll}0 & 1 \\ 1 & 0\end{array}\right)$, the map $\Phi: \mathcal{B}\left(\mathbb{C}^{2}\right) \rightarrow \mathcal{B}\left(\mathbb{C}^{2}\right)$ given by

$$
\Phi(\rho)=p \rho+(1-p) U(1) \rho U(1)^{*}, \quad \rho \in \mathcal{B}\left(\mathbb{C}^{2}\right),
$$

is a random unitary channel, known as the bit-fip channel. In the laboratory, it is one of the most common errors that can occur on a qubit via interaction with the environment [52] (see also [60, §8.3.3]), leaving a qubit invariant with probability $p$ and "flipping" a qubit with probability $1-p$. In our example above, the "flipping" corresponds to sending spin-up to spin-down, and vice versa.

From the harmonic analysis perspective, this channel arises from a locally compact group: its Kraus operators are precisely the left regular representation of $\mathbb{Z}_{2}$ ! As we shall see, our representation theorem 4.2.4 will shed new insight into this channel and many others by revealing their underlying algebraic structure. 


\section{Chapter 3}

\section{Locally Compact Quantum Groups}

In the attempt to generalize Pontrjagin duality to nonabelian groups, many partial results were established over half a century culminating in the early seventies with the theory of Kac algebras [21]. This wider category contains that of locally compact groups and provides the general framework necessary to establish duality.

Shortly afterwards, similar structures were appearing in algebra and mathematical physics, and the concept of a "quantum group" emerged. This resulted in many different axiomatic approaches, but from an abstract harmonic analysis perspective, the most natural are the von Neumann algebraic locally compact quantum groups, as developed by Kustermans and Vaes [50, 51]. This chapter is devoted to a brief introduction to this theory. We refer the reader to $[50,51,76]$ for details and proofs. 


\section{1 von Neumann Algebraic Quantum Groups}

Since the theory of von Neumann algebras may be viewed as noncommutative measure theory, these algebras are the perfect candidate for quantizing harmonic analysis.

Definition 3.1.1. A Hopf-von Neumann algebra is a pair $(M, \Gamma)$ where $M$ is a von Neumann algebra and $\Gamma: M \rightarrow M \bar{\otimes} M$ is a normal, unital, injective *-homomorphism satisfying the co-associativity condition $(\iota \otimes \Gamma) \Gamma=(\Gamma \otimes \iota) \Gamma$, that is, the following diagram

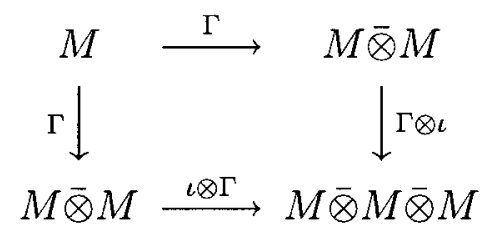

commutes. $\Gamma$ is then called a co-multiplication.

Given a Hopf-von Neumann algebra $(M, \Gamma)$, the preadjoint $\Gamma_{*}: M_{*} \widehat{\otimes} M_{*} \rightarrow M_{*}$ of the co-multiplication induces an associative completely contractive multiplication on $M_{*}$, turning it into a completely contractive Banach algebra.

Definition 3.1.2. A locally compact quantum group $\mathbb{G}$ is a quadruple $\mathbb{G}=(M, \Gamma, \varphi, \psi)$ where:

1. $(M, \Gamma)$ is a Hopf-von Neumann algebra;

2. $\varphi$ is an n.s.f. weight on $M$, called the left Haar weight, satisfying:

$$
\varphi((\omega \otimes \iota) \Gamma(x))=\omega(1) \varphi(x), \quad x \in \mathcal{M}_{\varphi}, \quad \omega \in M_{*} ;
$$


3. $\psi$ is an n.s.f. weight on $M$, called the right Haar weight, satisfying:

$$
\psi((\iota \otimes \omega) \Gamma(x))=\omega(1) \psi(x), \quad x \in \mathcal{M}_{\psi}, \quad \omega \in M_{*} .
$$

If $\mathbb{G}=(M, \Gamma, \varphi, \psi)$ is a locally compact quantum group, we say that $\mathbb{G}$ is commutative if $M$ is commutative, and co-commutative if $\Gamma$ is symmetric, that is, $\Sigma \circ \Gamma=\Gamma$ where $\Sigma: M \bar{\otimes} M \rightarrow M \bar{\otimes} M$ is the flip map given by $\Sigma(x \otimes y)=y \otimes x$. Two quantum groups $\mathbb{G}_{1}$ and $\mathbb{G}_{2}$ are isomorphic if there exists a von Neumann algebraic isomorphism $\alpha: M_{1} \rightarrow M_{2}$ such that $\Gamma_{2} \circ \alpha=(\alpha \otimes \alpha) \circ \Gamma_{1}$.

Example 3.1.3. Let $G$ be a locally compact group, and define $\Gamma_{a}: L^{\infty}(G) \rightarrow$ $L^{\infty}(G) \bar{\otimes} L^{\infty}(G) \cong L^{\infty}(G \times G)$ by

$$
\Gamma_{a}(f)(s, t)=f(s t), \quad s, t \in G
$$

Then $\Gamma_{a}$ is a co-multiplication which turns $\left(L^{\infty}(G), \Gamma_{a}\right)$ into a Hopf-von Neumann algebra. If $\varphi_{a}$ is the weight defined by the left Haar measure $d t$, then for $\omega \in L^{1}(G)=$ $L^{\infty}(G)_{*}$ and $f \in L^{\infty}(G) \cap L^{1}(G)=\mathcal{M}_{\varphi_{a}}$, we have

$$
\begin{aligned}
\varphi_{a}\left((\omega \otimes \iota) \Gamma_{a}(f)\right) & =\int_{G}(\omega \otimes \iota) \Gamma_{a}(f)(t) d t \\
& =\int_{G} \int_{G} \omega(s) f(s t) d s d t
\end{aligned}
$$


Since $f \in \mathcal{M}_{\varphi_{a}}$, we may apply Fubini's theorem to obtain:

$$
\begin{aligned}
\varphi_{a}\left((\omega \otimes \iota) \Gamma_{a}(f)\right) & =\int_{G} \omega(s) \int_{G} f(s t) d t d s \\
& =\int_{G} \omega(s) \int_{G} f(t) d t d s \\
& =\omega(1) \varphi_{a}(f)
\end{aligned}
$$

Thus, $\varphi_{a}$ is a left Haar weight on $L^{\infty}(G)$. Similarly, the weight $\psi_{a}$ given by the right Haar measure is a right Haar weight, and $\mathbb{G}_{a}=\left(L^{\infty}(G), \Gamma_{a}, \varphi_{a}, \psi_{a}\right)$ is a commutative locally compact quantum group. The co-multiplication $\Gamma_{a}$ is the dual of convolution in $L^{1}(G)$, viewed as a map $*: L^{1}(G) \widehat{\otimes} L^{1}(G) \rightarrow L^{1}(G)$.

As expected, we see that every locally compact group $G$ generates a "classical" locally compact quantum group given by the commutative von Neumann algebra $L^{\infty}(G)$. In fact, these are all such quantum groups.

Theorem 3.1.4. [77, §2] Let $\mathbb{G}=(M, \Gamma, \varphi, \psi)$ be a commutative locally compact quantum group. Then there exists a unique locally compact group $G$ for which $\mathbb{G} \cong$ $\mathbb{G}_{a}=\left(L^{\infty}(G), \Gamma_{a}, \varphi_{a}, \psi_{a}\right)$.

Example 3.1.5. Let $G$ be a locally compact group. Define $\Gamma_{s}: \mathcal{L}(G) \rightarrow \mathcal{L}(G) \bar{\otimes} \mathcal{L}(G)$ by

$$
\Gamma_{s}(\lambda(t))=\lambda(t) \otimes \lambda(t), \quad t \in G
$$

Then $\Gamma_{s}$ can be extended to a symmetric co-multiplication on $\mathcal{L}(G)$. It follows that there exists an n.s.f. weight $\varphi_{s}$ on $\mathcal{L}(G)$ (unique up to a positive scalar), namely 
Haagerup's Plancherel weight, which is both a left and a right Haar weight with respect to $\Gamma_{s}\left([73], \S\right.$ VII.3). Thus, $\mathbb{G}_{s}=\left(\mathcal{L}(G), \Gamma_{s}, \varphi_{s}\right)$ is a co-commutative locally compact quantum group. The co-multiplication $\Gamma_{s}$ is the dual of pointwise multiplication in $A(G)$, viewed as a map $\cdot: A(G) \widehat{\otimes} A(G) \rightarrow A(G)$.

Locally compact quantum groups therefore provide a sufficient framework for studying the duality of nonabelian groups, that is, the duality between $L^{\infty}(G)$ and $\mathcal{L}(G)$. Consequently, there is a dual version of Theorem 3.1.4. The proof can be established using theory we will encounter in $\S 3.3$, along with $[77, \S 2]$.

Theorem 3.1.6. Let $\mathbb{G}=(M, \Gamma, \varphi, \psi)$ be a co-commutative locally compact quantum group. Then there exists a unique locally compact group $G$ for which $\mathbb{G} \cong \mathbb{G}_{s}=$ $\left(\mathcal{L}(G), \Gamma_{s}, \varphi_{s}\right)$.

Motivated by the above examples, we may generalize various concepts from abstract harmonic analysis to the setting of locally compact quantum groups. Firstly, note that the existence of Haar weights is assumed in the definition of a locally compact quantum group. To find an axiomatization which yields the existence is a major open problem in quantum group theory. Despite this fact, assuming existence is enough to guarantee uniqueness.

Theorem 3.1.7. [76, §1.10] The left and right Haar weights on a locally compact quantum group are unique up to positive scalars. 
Remark 3.1.8. Under the GNS construction of the left (respectively, right) Haar weight, we may identify $M$ with a subalgebra in $\mathcal{B}\left(H_{\varphi}\right)$ (respectively, $\mathcal{B}\left(H_{\psi}\right)$ ) and we will do so without comment throughout the thesis.

For notational symmetry, given a locally compact quantum group $\mathbb{G}=(M, \Gamma, \varphi, \psi)$, we write $L^{\infty}(\mathbb{G}):=M$ and $L^{1}(\mathbb{G}):=M_{*}$. In the commutative (respectively, cocommutative) setting we simply have $L^{\infty}\left(\mathbb{G}_{a}\right)=L^{\infty}(G)$ and $L^{1}\left(\mathbb{G}_{a}\right)=L^{1}(G)$ (respectively, $L^{\infty}\left(\mathbb{G}_{s}\right)=\mathcal{L}(G)$ and $\left.L^{1}\left(\mathbb{G}_{s}\right)=A(G)\right)$ for a locally compact group $G$.

Definition 3.1.9. A locally compact quantum group $\mathbb{G}=(M, \Gamma, \varphi, \psi)$ is said to be:

- discrete if $L^{1}(\mathbb{G})$ is a unital Banach algebra;

- compact if $\varphi$ is finite;

- amenable if there exists a state $F$ on $L^{\infty}(\mathbb{G})$ such that

$$
\langle(f \otimes \iota) \Gamma(x), F\rangle=\langle(\iota \otimes f) \Gamma(x), F\rangle=\langle f, 1\rangle\langle x, F\rangle
$$

for all $f \in L^{1}(\mathbb{G}), x \in L^{\infty}(\mathbb{G})$;

- co-amenable if $L^{1}(\mathbb{G})$ has a bounded approximate identity.

In what follows, we will refrain from writing the quadruple notation for a locally compact quantum group and simply write $\mathbb{G}$, where the usual notations for the comultiplication and Haar weights will be understood. 


\subsection{The Fundamental Unitary}

Given a locally compact group $G$, there is a fundamental unitary operator $W_{a}$ : $L^{2}(G \times G) \rightarrow L^{2}(G \times G)$ given by

$$
W_{a} \xi(s, t)=\xi\left(s, s^{-1} t\right), \quad s, t \in G,
$$

for all $\xi \in L^{2}(G \times G) \cong L^{2}(G) \otimes_{2} L^{2}(G)$, where $\otimes_{2}$ denotes the Hilbert space tensor product. We use the adjective "fundamental" as this unitary carries nearly all the information of $G$ along with its corresponding quantum group $\mathbb{G}_{a}$ in the following sense:

1. $\Gamma_{a}\left(M_{f}\right)=W_{a}^{*}\left(1 \otimes M_{f}\right) W_{a}, \quad f \in L^{\infty}(G)$;

2. $W_{a} \in L^{\infty}(G) \bar{\otimes} \mathcal{L}(G)=L^{\infty}(G) \bar{\otimes} \mathcal{R}(G)^{\prime}$;

3. $L^{\infty}(G)={\overline{\left\{(\iota \otimes \psi) W_{a}: \psi \in A(G)\right.}}^{w^{*}}$;

4. $C_{0}(G)={\overline{\left\{(\iota \otimes \psi) W_{a}: \psi \in A(G)\right.}}^{\|\cdot\|}$;

5. $\lambda(f)=(f \otimes \iota)\left(W_{a}\right), \quad f \in L^{1}(G)$

6. $\mathcal{L}(G)={\overline{\left\{(f \otimes \iota) W_{a}: f \in L^{1}(G)\right.}}^{w^{*}}$;

7. $\left.C_{\lambda}^{*}(G)=\overline{\left\{(f \otimes \iota) W_{a}: f \in L^{1}(G)\right.}\right\}^{\|\cdot\|}$. 
In general, given a locally compact quantum group $\mathbb{G}$, there exists a unitary operator $W: H_{\varphi} \otimes_{2} H_{\varphi} \rightarrow H_{\varphi} \otimes_{2} H_{\varphi}$ satisfying

$$
W^{*}\left(\Lambda_{\varphi}(x) \otimes \Lambda_{\varphi}(y)\right)=\left(\Lambda_{\varphi} \otimes \Lambda_{\varphi}\right)(\Gamma(y)(x \otimes 1)), \quad x, y \in \mathcal{N}_{\varphi}
$$

This operator satisfies the pentagonal relation, that is

$$
W_{12} W_{13} W_{23}=W_{23} W_{12}
$$

where $W_{12}=W \otimes 1, W_{23}=1 \otimes W, W_{13}=(\sigma \otimes 1) W_{23}(\sigma \otimes 1)$ and $\sigma$ is the flip map on $H_{\varphi} \otimes_{2} H_{\varphi}$. The map $W$ is therefore called a multiplicative unitary, and similarly to the classical case above, it carries nearly all the information about $\mathbb{G}$. In particular, the co-multiplication on $L^{\infty}(\mathbb{G})$ may be written as

$$
\Gamma(x)=W^{*}(1 \otimes x) W, \quad x \in L^{\infty}(\mathbb{G})
$$

The operator $W$ will be called the left fundamental unitary of $\mathbb{G}$. It may also be used to extend $\Gamma$ to $\tilde{\Gamma}: \mathcal{B}\left(H_{\varphi}\right) \rightarrow L^{\infty}(\mathbb{G}) \bar{\otimes} \mathcal{B}\left(H_{\varphi}\right)$ via the same formula:

$$
\tilde{\Gamma}(x)=W^{*}(1 \otimes x) W, \quad x \in \mathcal{B}\left(H_{\varphi}\right)
$$


Definition 3.2.1. The left regular representation $\lambda: L^{1}(\mathbb{G}) \rightarrow \mathcal{B}\left(H_{\varphi}\right)$ of $\mathbb{G}$ is defined by

$$
\lambda(f)=(f \otimes \iota)(W), \quad f \in L^{1}(\mathbb{G}) .
$$

Using the properties of $W$, it can be shown that $\lambda$ is an injective completely contractive homomorphism.

In a similar fashion, there is a right fundamental unitary operator $V: H_{\psi} \otimes_{2} H_{\psi} \rightarrow$ $H_{\psi} \otimes_{2} H_{\psi}$ defined by

$$
V\left(\Lambda_{\psi}(x) \otimes \Lambda_{\psi}(y)\right)=\left(\Lambda_{\psi} \otimes \Lambda_{\psi}\right)(\Gamma(x)(1 \otimes y)), \quad x, y, \in \mathcal{N}_{\psi}
$$

It can be shown that $V$ also satisfies the pentagonal relation and that the co-multiplication on $L^{\infty}(\mathbb{G})$ may be written as

$$
\Gamma(x)=V(x \otimes 1) V^{*}, \quad x \in L^{\infty}(\mathbb{G}) .
$$

We also obtain the right regular representation $\rho: L^{1}(\mathbb{G}) \rightarrow \mathcal{B}\left(H_{\psi}\right)$, given by

$$
\rho(f)=(\iota \otimes f)(V), \quad f \in L^{1}(\mathbb{G})
$$




\subsection{Duality}

One of the many triumphs of Fourier analysis on locally compact abelian groups provided the following isomorphisms:

$$
L^{\infty}(G) \cong \mathcal{L}(\hat{G}), \quad L^{1}(G) \cong A(\hat{G}), \quad \text { and } \quad L^{2}(G) \cong L^{2}(\hat{G})
$$

In order to achieve a successful generalization, we must therefore ensure the duality of $\mathbb{G}_{a}=\left(L^{\infty}(G), \Gamma_{a}, \varphi_{a}, \psi_{a}\right)$ and $\mathbb{G}_{s}=\left(\mathcal{L}(G), \Gamma_{s}, \varphi_{s}\right)$ for all locally compact groups. This may be done with the aid of the fundamental unitaries introduced above.

Theorem 3.3.1 (The Dual Quantum Group). [76, $§ 1.11]$ Let $\mathbb{G}$ be a locally compact quantum group with left fundamental unitary $W$. Define $L^{\infty}(\hat{\mathbb{G}})=\{\lambda(f): f \in$ $\left.L^{1}(\mathbb{G})\right\}^{\prime \prime}$. Then $\hat{\Gamma}: L^{\infty}(\hat{\mathbb{G}}) \rightarrow L^{\infty}(\hat{\mathbb{G}}) \bar{\otimes} L^{\infty}(\hat{\mathbb{G}})$ given by

$$
\hat{\Gamma}(\hat{x})=\hat{W}^{*}(1 \otimes \hat{x}) \hat{W}, \quad \hat{x} \in L^{\infty}(\hat{\mathbb{G}}),
$$

where $\hat{W}=\sigma W^{*} \sigma$, is a co-multiplication on $L^{\infty}(\hat{\mathbb{G}})$. Furthermore, there exists a left Haar weight $\hat{\varphi}$ and a right Haar weight $\hat{\psi}$ on $L^{\infty}(\hat{\mathbb{G}})$ such that $\hat{\mathbb{G}}=\left(L^{\infty}(\hat{\mathbb{G}}), \hat{\Gamma}, \hat{\varphi}, \hat{\psi}\right)$ is a locally compact quantum group.

As a consequence, it follows that $W \in L^{\infty}(\mathbb{G}) \bar{\otimes} L^{\infty}(\hat{\mathbb{G}})$ and $H_{\varphi} \cong H_{\hat{\varphi}}$. Hence the dual (left) regular representation

$$
\hat{\lambda}: L^{1}(\hat{\mathbb{G}}) \ni \hat{f} \mapsto(\hat{f} \otimes \iota)(\hat{W})=(\iota \otimes \hat{f})\left(W^{*}\right) \in L^{\infty}(\mathbb{G})
$$


generates a von Neumann subalgebra $L^{\infty}(\hat{\hat{\mathbb{G}}}):=\left\{\hat{\lambda}(\hat{f}): \hat{f} \in L^{1}(\hat{\mathbb{G}})\right\}^{\prime \prime}$ of $L^{\infty}(\mathbb{G})$. In fact, we have a perfect duality.

Theorem 3.3.2 (Quantum Pontrjagin Duality). [76, §1.11] Let $\mathbb{G}$ be a locally compact quantum group. Then

$$
\hat{\hat{\mathbb{G}}} \cong \mathbb{G}
$$

By considering the right regular representation, we obtain the corresponding dual quantum group $\hat{\mathbb{G}}^{\prime}=\left(L^{\infty}\left(\hat{\mathbb{G}}^{\prime}\right), \hat{\Gamma}^{\prime}, \hat{\varphi}^{\prime}, \hat{\psi}^{\prime}\right)$, where the co-multiplication $\hat{\Gamma}^{\prime}$ is defined by

$$
\hat{\Gamma}^{\prime}\left(\hat{x}^{\prime}\right)=\hat{V}\left(\hat{x}^{\prime} \otimes 1\right) \hat{V}^{*}, \quad \hat{x}^{\prime} \in L^{\infty}\left(\hat{\mathbb{G}}^{\prime}\right),
$$

and $\hat{V}=\sigma V^{*} \sigma$. In this case (by considering the duality between $\mathbb{G}$ and $\hat{\mathbb{G}}^{\prime}$ ), we obtain $\hat{V} \in L^{\infty}(\mathbb{G}) \bar{\otimes} L^{\infty}\left(\hat{\mathbb{G}}^{\prime}\right)$ and therefore $V \in L^{\infty}\left(\hat{\mathbb{G}}^{\prime}\right) \bar{\otimes} L^{\infty}(\mathbb{G})$.

As noted above, the Hilbert spaces in the GNS-constructions of the left Haar weights on $\mathbb{G}$ and $\hat{\mathbb{G}}$ may be identified. The analogous result holds for the right Haar weights, and it follows that $H_{\varphi} \cong H_{\hat{\varphi}} \cong H_{\psi} \cong H_{\hat{\psi}}$. We will therefore denote this common Hilbert space by $L^{2}(\mathbb{G})$.

By analogy with the classical setting, we define

$$
C_{0}(\mathbb{G}):={\overline{\hat{\lambda}}\left(L^{1}(\hat{\mathbb{G}})\right)}^{\|\cdot\|}
$$

Then $C_{0}(\mathbb{G})$ is a weak* dense $C^{*}$-subalgebra of $L^{\infty}(\mathbb{G})$, called the reduced quantum group associated to $\mathbb{G}$. We define $M(\mathbb{G})$ to be the operator dual $C_{0}(\mathbb{G})^{*}$. Then we 
have $C_{0}\left(\mathbb{G}_{a}\right)=C_{0}(G)$ and $M\left(\mathbb{G}_{a}\right)=M(G)$.

Example 3.3.3. If $G$ is a locally compact group, $\mathbb{G}_{a}=\left(L^{\infty}(G), \Gamma_{a}, \varphi_{a}, \psi_{a}\right)$ and $\mathbb{G}_{s}=$ $\left(\mathcal{L}(G), \Gamma_{s}, \varphi_{s}\right)$ are dual objects in the category of locally compact quantum groups, therefore establishing Pontrjagin duality for nonabelian locally compact groups.

A beautiful consequence of this duality is given by the following proposition, known as Heisenberg's theorem.

Proposition 3.3.4. Let $G$ be a locally compact group. Then

$$
L^{\infty}(G) \cap \mathcal{L}(G)=\mathbb{C} 1=L^{\infty}(G) \cap \mathcal{R}(G)
$$

Equivalently, $L^{\infty}(G) \vee \mathcal{L}(G)=\mathcal{B}\left(L^{2}(G)\right)=L^{\infty}(G) \vee \mathcal{R}(G)$, where $L^{\infty}(G) \vee \mathcal{L}(G)$ denotes the von Neumann algebra generated by $L^{\infty}(G)$ and $\mathcal{L}(G)$.

Proof. If $f \in L^{\infty}(G)$ and $s \in G$, then $\lambda(s) M_{f}=M_{\lambda(s) f} \lambda(s)$. Thus, if $M_{f} \in L^{\infty}(G) \cap$ $\mathcal{R}(G)$ then

$$
M_{\lambda(s) f}=\lambda(s) M_{f} \lambda(s)^{*}=M_{f}
$$

for all $s \in G$, which implies that $f$ is a constant function. Hence, $L^{\infty}(G) \cap \mathcal{R}(G)=\mathbb{C} 1$. Taking the commutant, we obtain

$$
\mathcal{B}\left(L^{2}(G)\right)=(\mathbb{C} 1)^{\prime}=\left(L^{\infty}(G) \cap \mathcal{R}(G)\right)^{\prime}=L^{\infty}(G) \vee \mathcal{L}(G)
$$


The following two propositions constitute the generalization of Heisenberg's theorem to the noncommutative setting. The notation $\left\langle L^{\infty}(\mathbb{G}) L^{\infty}(\hat{\mathbb{G}})\right\rangle$ refers to the weak* closed linear span of $L^{\infty}(\mathbb{G}) L^{\infty}(\hat{\mathbb{G}})=\left\{x \hat{x}: x \in L^{\infty}(\mathbb{G}), \hat{x} \in L^{\infty}(\hat{\mathbb{G}})\right\}$.

Proposition 3.3.5. [79] Let $\mathbb{G}$ be a locally compact quantum group. Then

$$
\begin{aligned}
\mathbb{C} 1 & =L^{\infty}(\mathbb{G}) \cap L^{\infty}(\hat{\mathbb{G}})=L^{\infty}(\mathbb{G})^{\prime} \cap L^{\infty}(\hat{\mathbb{G}}) \\
& =L^{\infty}(\mathbb{G}) \cap L^{\infty}\left(\hat{\mathbb{G}}^{\prime}\right)=L^{\infty}(\mathbb{G})^{\prime} \cap L^{\infty}\left(\hat{\mathbb{G}}^{\prime}\right) .
\end{aligned}
$$

Proposition 3.3.6. [78, Proposition 2.5] Let $\mathbb{G}$ be a locally compact quantum group. Then

$$
\begin{aligned}
\mathcal{B}\left(L^{2}(\mathbb{G})\right) & =\left\langle L^{\infty}\left(\hat{\mathbb{G}}^{\prime}\right) L^{\infty}(\hat{\mathbb{G}})\right\rangle=\left\langle L^{\infty}(\hat{\mathbb{G}}) L^{\infty}(\mathbb{G})^{\prime}\right\rangle \\
& =\left\langle L^{\infty}(\mathbb{G}) L^{\infty}(\hat{\mathbb{G}})\right\rangle=\left\langle L^{\infty}(\mathbb{G})^{\prime} L^{\infty}\left(\hat{\mathbb{G}}^{\prime}\right)\right\rangle .
\end{aligned}
$$

\subsection{The Antipode}

The antipode is the quantum analogue of group inversion. Since inversion is the distinguishing feature between groups and semi-groups, it is not surprising that its generalization to quantum groups plays a vital role in the theory. For motivation, let us consider the commutative case.

If $G$ is a locally compact group, define $S: L^{\infty}(G) \rightarrow L^{\infty}(G)$ by $S(f)(s)=f\left(s^{-1}\right)$, 
$s \in G$. We may also define a corresponding right Haar weight $\psi$ on $L^{\infty}(G)$ by

$$
\psi(f)=\int_{G} f\left(s^{-1}\right) d s, \quad f \in L^{\infty}(G)
$$

where $d s$ denotes a fixed left Haar measure on $G$. In terms of the co-multiplication on $L^{\infty}(G)$, it follows that

$$
S\left((\psi \otimes \iota)\left((f \otimes 1) \Gamma_{a}(g)\right)\right)=(\psi \otimes \iota)\left(\Gamma_{a}(f)(g \otimes 1)\right), \quad f, g \in L^{\infty}(G) .
$$

Indeed,

$$
\begin{aligned}
S\left((\psi \otimes \iota)\left((f \otimes 1) \Gamma_{a}(g)\right)\right)(s) & =(\psi \otimes \iota)\left((f \otimes 1) \Gamma_{a}(g)\right)\left(s^{-1}\right) \\
& =\int_{G}(f \otimes 1) \Gamma_{a}(g)\left(t^{-1}, s^{-1}\right) d t \\
& =\int_{G} f\left(t^{-1}\right) g\left(t^{-1} s^{-1}\right) d t \\
& =\int_{G} f\left(t^{-1} s\right) g\left(t^{-1}\right) d t \\
& =(\psi \otimes \iota)\left(\Gamma_{a}(f)(g \otimes 1)\right)(s) .
\end{aligned}
$$

In order to obtain a similar formula for general $\mathbb{G}$, we begin at the level of the Hilbert space $L^{2}(\mathbb{G})$ on which it acts. 
Proposition 3.4.1. [76, Propositıon 1.14.3] For every $x, y \in \mathcal{N}_{\varphi}^{*} \mathcal{N}_{\psi}$, we have $(\psi \otimes \iota)\left(\Gamma\left(x^{*}\right)(y \otimes 1)\right) \in \mathcal{N}_{\varphi}$. If we define

$$
G \Lambda_{\varphi}\left((\psi \otimes \iota)\left(\Gamma\left(x^{*}\right)(y \otimes 1)\right)\right)=\Lambda_{\varphi}\left((\psi \otimes \iota)\left(\Gamma\left(y^{*}\right)(x \otimes 1)\right)\right)
$$

then $G$ ıs a closed densely defined antı-linear operator on $L^{2}(\mathbb{G})$. Furthermore, if $I N^{\frac{1}{2}}$ us its polar decomposition, then

1. $I=I^{*}$;

2. $I^{2}=1$;

3. $I N I=N^{-1}$.

Defining the operators

$$
\begin{aligned}
& R: L^{\infty}(\mathbb{G}) \ni x \mapsto I x^{*} I \in L^{\infty}(\mathbb{G}) ; \\
& \tau_{t}: L^{\infty}(\mathbb{G}) \ni x \mapsto N^{-\imath t} x N^{\imath t} \in L^{\infty}(\mathbb{G}), \quad t \in \mathbb{R},
\end{aligned}
$$

it follows that $R$ is an anti-automorphism on $L^{\infty}(\mathbb{G})$ such that $R^{2}=1$, and $\left(\tau_{t}\right)_{t \in \mathbb{R}}$ is a strongly continuous one-parameter group of automorphisms on $L^{\infty}(\mathbb{G})$. We call $R$ the unitary antipode of $\mathbb{G}$, and $\left(\tau_{t}\right)_{t \in \mathbb{R}}$ the scalıng group of $\mathbb{G}$. Note that $\tau_{t} R=R \tau_{t}$ for all $t \in \mathbb{R}$ since $I N I=N^{-1}$.

Definition 3.4.2. The antrpode of $\mathbb{G}$ is defined as $S=R \tau_{-\frac{2}{2}}$, where $\tau_{-\frac{2}{2}}$ is the analytic extension of $\left(\tau_{t}\right)_{t \in \mathbb{R}}$ at $z=-\frac{\imath}{2} \in \mathbb{C}$. We let $\mathcal{D}(S)$ denote its domain. 
Theorem 3.4.3. [76, §1.4] The antipode $S$ has the following properties:

1. $S$ is densely defined and has dense range;

2. $S$ is injective and $S^{-1}=R \tau_{\frac{2}{2}}=\tau_{\frac{2}{2}} R$;

3. $S$ is anti-multiplicative, that is, for all $x, y \in \mathcal{D}(S), x y \in \mathcal{D}(S)$ and $S(x y)=$ $S(y) S(x)$

4. for all $x \in \mathcal{D}(S), S(x)^{*} \in \mathcal{D}(S)$ and $S\left(S(x)^{*}\right)^{*}=x$;

5. for every $x, y \in \mathcal{N}_{\psi}$, we have $(\psi \otimes \iota)\left(\left(x^{*} \otimes 1\right) \Gamma(y)\right) \in \mathcal{D}(S)$ and

$$
S\left((\psi \otimes \iota)\left(\left(x^{*} \otimes 1\right) \Gamma(y)\right)\right)=(\psi \otimes \iota)\left(\Gamma\left(x^{*}\right)(y \otimes 1)\right)
$$

6. $\mathcal{D}(S)$ contains $(\iota \otimes \hat{f})(W)$ for all $\hat{f} \in L^{1}(\hat{\mathbb{G}})$, and

$$
S((\iota \otimes \hat{f})(W))=(\iota \otimes \hat{f})\left(W^{*}\right)
$$

\subsection{Quantum Groups in the Universal Setting}

In the classical setting one uses the antipode $S$ to define an involution on $L^{1}(G)=$ $L^{1}\left(\hat{\mathbb{G}}_{s}\right)$, whose enveloping $C^{*}$-algebra $C^{*}(G)$ plays an important role in abstract harmonic analysis. For instance, the representation theory of $G$ is equivalent to that 
of $C^{*}(G)$, and for abelian groups, $C^{*}(G)$ is isometrically $*$-isomorphic to $C_{0}(\hat{G})[25$, $\S 7.1]$.

For general locally compact quantum groups, one would like an analogue of the group $C^{*}$-algebra. Unfortunately, the straightforward generalization to define an involution on $L^{1}(\hat{\mathbb{G}})$ respected by the left regular representation is not possible as the antipode $S$ may be unbounded. Instead, one defines an involution on a dense subalgebra of $L^{1}(\hat{\mathbb{G}})$, then passes to its enveloping $C^{*}$-algebra in the same manner as in the classical case. The resulting object is known as the universal quantum group associated to $\mathbb{G}$, and we outline its construction below; for details, see [48].

Let $\mathbb{G}$ be a locally compact quantum group with dual $\hat{\mathbb{G}}$, and let $L_{*}^{1}(\hat{\mathbb{G}})$ be the subspace of $L^{1}(\hat{\mathbb{G}})$ defined by

$$
L_{*}^{1}(\hat{\mathbb{G}})=\left\{\hat{f} \in L^{1}(\hat{\mathbb{G}}): \exists \hat{g} \in L^{1}(\hat{\mathbb{G}}) \quad \text { s.t. } \quad \hat{g}(\hat{x})=\hat{f}^{*} \circ \hat{S}(\hat{x}) \quad \forall \hat{x} \in \mathcal{D}(\hat{S})\right\}
$$

where $\hat{f}^{*}(\hat{x})=\overline{\hat{f}\left(\hat{x}^{*}\right)}, \hat{x} \in L^{\infty}(\hat{\mathbb{G}})$. It is known from $[76, \S 1.13]$ that $L_{*}^{1}(\hat{\mathbb{G}})$ is a dense subalgebra of $L^{1}(\hat{\mathbb{G}})$. There is an involution on $L_{*}^{1}(\hat{\mathbb{G}})$ given by $\hat{f}^{o}=\hat{f}^{*} \circ \hat{S}$, such that $L_{*}^{1}(\hat{\mathbb{G}})$ becomes a Banach $*$-algebra under the norm

$$
\|\hat{f}\|_{*}=\max \left\{\|\hat{f}\|,\left\|\hat{f}^{o}\right\|\right\}
$$

Moreover, the restricted left regular representation $\hat{\lambda}: L_{*}^{1}(\hat{\mathbb{G}}) \rightarrow C_{0}(\mathbb{G})$ becomes a *-homomorphism. Indeed, by Theorem 3.4.3, the domain of $\hat{S}$ contains $(\iota \otimes f)(\hat{W})$, 
$f \in L^{1}(\mathbb{G})$, and since $\hat{S}((\iota \otimes f)(\hat{W}))=(\iota \otimes f)\left(\hat{W}^{*}\right)$, we have

$$
\begin{aligned}
\left\langle\hat{\lambda}\left(\hat{f}^{o}\right), f\right\rangle & =\left\langle\left(\hat{f}^{o} \otimes \iota\right)(\hat{W}), f\right\rangle \\
& =\left\langle\hat{f}^{o},(\iota \otimes f)(\hat{W})\right\rangle \\
& =\left\langle\hat{f}^{*},(\iota \otimes f)\left(\hat{W}^{*}\right)\right\rangle \\
& =\left\langle\left(\hat{f}^{*} \otimes \iota\right)\left(\hat{W}^{*}\right), f\right\rangle \\
& =\left\langle[(\hat{f} \otimes \iota)(\hat{W})]^{*}, f\right\rangle \\
& =\left\langle\hat{\lambda}(\hat{f})^{*}, f\right\rangle,
\end{aligned}
$$

where in the second last step we have used the normality of the linear functional $\left(\hat{f}^{*} \otimes \iota\right): L^{\infty}(\hat{\mathbb{G}}) \bar{\otimes} L^{\infty}(\mathbb{G}) \rightarrow L^{\infty}(\mathbb{G})$, together with the weak* continuity of the adjoint.

Next, define the enveloping $C^{*}$-algebra by introducing the following norm for all $\hat{f} \in L_{*}^{1}(\hat{\mathbb{G}})$,

$$
\|\hat{f}\|_{u}=\sup \left\{\|\pi(\hat{f})\| \mid \pi: L_{*}^{1}(\hat{\mathbb{G}}) \rightarrow \mathcal{B}(H) \text { is a s.nd. *-representation }\right\}
$$

Taking the completion, we obtain the universal quantum group $C^{*}$-algebra of $\mathbb{G}$, denoted $C_{u}(\mathbb{G})$. Writing $\hat{\lambda}_{u}$ for the embedding of $L_{*}^{1}(\hat{\mathbb{G}})$ into $C_{u}(\mathbb{G})$, the pair $\left(C_{u}(\mathbb{G}), \hat{\lambda}_{u}\right)$ is determined (up to $*$-isomorphism) by the following universal property: If $\mathcal{A}$ is a $C^{*}$-algebra and $\pi: L_{*}^{1}(\hat{\mathbb{G}}) \rightarrow \mathcal{A}$ is a $*$-representation, then there exists a unique *-representation $\pi_{u}: C_{u}(\mathbb{G}) \rightarrow \mathcal{A}$ such that $\pi_{u} \circ \hat{\lambda}_{u}=\pi$. 
For commutative quantum groups $\mathbb{G}_{a}$, we have $C_{u}\left(\mathbb{G}_{a}\right)=C_{0}(G)$ and for cocommutative quantum groups $\mathbb{G}_{s}$, we obtain $C_{u}\left(\mathbb{G}_{s}\right)=C^{*}(G)$, the full group $C^{*}$ algebra of $G$, thereby making $C_{u}(\mathbb{G})$ the appropriate generalization to the quantum group setting.

Definition 3.5.1. Let $\mathcal{A}$ be a $C^{*}$-algebra. The idealizer of $\mathcal{A}$ in $\left(\mathcal{A}^{* *}, \square\right)$, denoted $M(\mathcal{A})$, is defined by

$$
\left\{x \in \mathcal{A}^{* *}: x \square a, a \square x \in \mathcal{A} \text { for all } a \in \mathcal{A}\right\}
$$

and is called the multiplier algebra of $\mathcal{A}$.

Note that $M(\mathcal{A})$ is a $C^{*}$-subalgebra of $\mathcal{A}^{* *}$, containing $\mathcal{A}$ as a norm-closed two sided ideal, and $\mathcal{A}$ is unital if and only if $M(\mathcal{A})=\mathcal{A}$. Moreover, if $\mathcal{A}$ is a $C^{*}$ subalgebra of $\mathcal{B}(H)$ for a Hilbert space $H$, then $M(\mathcal{A}) \subseteq \mathcal{B}(H)[62$, Proposition 2.12.9]. For instance, if $\mathcal{A}=\mathcal{K}(H)$, the $C^{*}$-algebra of compact operators $H$, then $M(\mathcal{A})=\mathcal{B}(H)$. For details on multiplier algebras, see $[62, \S 2.12]$.

Definition 3.5.2. Let $\mathcal{A}$ and $\mathcal{B}$ be $C^{*}$-algebras. For every $a \in \mathcal{A}$, define a semi-norm on $M(\mathcal{A})$ by

$$
\|x\|_{a}=\|x \square a\|+\|a \square x\|, \quad x \in M(\mathcal{A}) .
$$

The weak topology generated by the semi-norms $\left\{\|\cdot\|_{a}: a \in \mathcal{A}\right\}$ is called the strict topology in $M(\mathcal{A})$. We say that a linear map $\pi: \mathcal{A} \rightarrow M(\mathcal{B})$ is strict if it is norm bounded and strictly continuous on bounded sets. 
One can show that if $\pi: \mathcal{A} \rightarrow M(\mathcal{B})$ is strict, then it has a unique bounded linear extension $\tilde{\pi}: M(\mathcal{A}) \rightarrow M(\mathcal{B})$ which is strictly continuous on bounded sets $[49, \S 7]$. Three examples of importance are bounded linear functionals $\varphi \in \mathcal{A}^{*}$, their associated slice maps

$$
\varphi \otimes \iota: \mathcal{A} \otimes_{m \imath n} \mathcal{B} \rightarrow M(\mathcal{B}) \quad \text { and } \quad \iota \otimes \varphi: \mathcal{B} \otimes_{m \imath n} \mathcal{A} \rightarrow M(\mathcal{B}), \quad \varphi \in \mathcal{A}^{*}
$$

and nondegenerate $*$-representations $\pi: \mathcal{A} \rightarrow M(\mathcal{B})$, meaning

$$
\mathcal{B}=\overline{\{\pi(a) \square b: a \in \mathcal{A}, \quad b \in \mathcal{B}\}}^{\|\cdot\|} .
$$

In what follows, we will refrain from writing $\tilde{\pi}$ for the extensions of strict linear mappings, and simply write $\pi$.

The multiplier algebra of $C_{0}(\mathbb{G})$ plays a prominent role in the $C^{*}$-algebraic formulation of locally compact quantum groups. For instance, $\left.\Gamma\right|_{C_{0}(\mathbb{G})}: C_{0}(\mathbb{G}) \rightarrow$ $M\left(C_{0}(\mathbb{G}) \otimes_{\min } C_{0}(\mathbb{G})\right)$ is the co-multiplication on the reduced quantum group $C_{0}(\mathbb{G})$ [4]. This allows one to construct a multiplication on $M(\mathbb{G})$ via

$$
\langle\mu \star \nu, x\rangle=\langle\mu \otimes \nu, \Gamma(x)\rangle, \quad x \in C_{0}(\mathbb{G}), \quad \mu, \nu \in M(\mathbb{G})
$$

where $\mu \otimes \nu \in M\left(C_{0}(\mathbb{G}) \otimes_{m \imath n} C_{0}(\mathbb{G})\right)^{*}$ is understood as the composition $\mu \circ(\iota \otimes \nu)$. Under $\star$, the space $M(\mathbb{G})$ becomes a completely contractive Banach algebra containing $L^{1}(\mathbb{G})$ as a norm closed two-sided ideal via the map $\left.L^{1}(\mathbb{G}) \ni f \mapsto f\right|_{C_{0}(\mathbb{G})} \in M(\mathbb{G})$. 
Since $\hat{\lambda}: L_{*}^{1}(\hat{\mathbb{G}}) \rightarrow C_{0}(\mathbb{G})$ is a $*$-homomorphism, by the universal property of $C_{u}(\mathbb{G})$, there exists a unique surjective $*$-homomorphism $\pi: C_{u}(\mathbb{G}) \rightarrow C_{0}(\mathbb{G})$ such that $\pi \circ \hat{\lambda}_{u}(\hat{f})=\hat{\lambda}(\hat{f})$ for all $\hat{f} \in L_{*}^{1}(\hat{\mathbb{G}})$. Furthermore, there is a nondegenerate co-associative $*$-homomorphism $\Gamma_{u}: C_{u}(\mathbb{G}) \rightarrow M\left(C_{u}(\mathbb{G}) \otimes_{\min } C_{u}(\mathbb{G})\right)$ satisfying

$$
(\pi \otimes \pi) \circ \Gamma_{u}=\Gamma \circ \pi
$$

see [48, Proposition 6.2]. The space $C_{u}(\mathbb{G})^{*}$ then has the structure of a unital completely contractive Banach algebra via

$$
\left\langle\mu \star_{u} \nu, x\right\rangle=\left\langle\mu \otimes \nu, \Gamma_{u}(x)\right\rangle, \quad x \in C_{u}(\mathbb{G}), \quad \mu, \nu \in C_{u}(\mathbb{G})^{*}
$$

Under this multiplication, the map $i: L^{1}(\mathbb{G}) \rightarrow C_{u}(\mathbb{G})^{*}$ given by the composition of the inclusion $L^{1}(\mathbb{G}) \subseteq M(\mathbb{G})$ and $\pi^{*}: M(\mathbb{G}) \rightarrow C_{u}(\mathbb{G})^{*}$ is an injective homomorphism. It follows that $i\left(L^{1}(\mathbb{G})\right)$ is a norm closed two-sided ideal in $C_{u}(\mathbb{G})^{*}[48$, Proposition 8.3]. This result allows us to view the elements of $C_{u}(\mathbb{G})^{*}$ as completely bounded multipliers of $\mathbb{G}$, and as we shall see, the positive elements in $C_{u}(\mathbb{G})^{*}$ will be our main tool for generating quantum channels from locally compact quantum groups. 


\section{Chapter 4}

\section{Completely Bounded Multipliers}

This chapter contains the main theoretical results of the thesis and is largely based on the Junge-Neufang-Ruan representation theorem in [41]. In their paper they define the left (respectively, right) completely bounded multiplier algebra of $L^{1}(\mathbb{G})$, which can be viewed as a quantization of the measure algebra of a locally compact group. The modern representation framework they use has an interesting history and incorporates the theory of operator spaces in an essential way. We therefore begin this chapter with a brief introduction to the new framework and the main results concerning representations of group algebras.

\subsection{Introduction}

The framework utilized in [41] is motivated by the following question. It is well known that for every locally compact group $G$, we may isometrically represent $C_{0}(G)$ inside 
$\mathcal{B}(H)$ for some Hilbert space $H$. Can a similar representation be found for the dual $M(G) ?$

It was shown by Ghahramani [26] that if $G$ contains at least two elements, the convolution algebra $L^{1}(G)$ (and thus the measure algebra) cannot be isometrically isomorphic to a subalgebra of operators on any Hilbert space. Hence, the representation of the measure algebra must be considered outside the realm of Hilbert spaces.

The first such representation came from Wendel [82] in 1952. He showed that $M(G)$ is isometrically isomorphic to the right centralizer algebra $C^{r}\left(L^{1}(G)\right)$ of $L^{1}(G)$, i.e., those bounded maps on $\Phi$ on $L^{1}(G)$ satisfying $\Phi(f * g)=f * \Phi(g), f, g \in L^{1}(G)$. Indeed, he showed that every measure $\mu \in M(G)$ corresponds uniquely to a bounded right centralizer

$$
m_{\mu}: L^{1}(G) \ni f \mapsto f * \mu \in L^{1}(G)
$$

The adjoint $\Phi_{\mu}=m_{\mu}^{*}$ then defines a (bounded) weak* continuous operator on $L^{\infty}(G)$ commuting with left translations,

$$
\Phi_{\mu}(f)(s)=\mu * f(s)=\int_{G} f\left(t^{-1} s\right) d \mu(t), \quad f \in L^{\infty}(G), s \in G
$$

Moreover, every such operator on $L^{\infty}(G)$ is implemented by a measure in this fashion, and we obtain an isometric isomorphism

$$
\Phi: M(G) \ni \mu \mapsto \Phi_{\mu} \in \mathcal{B}_{l}^{\sigma}\left(L^{\infty}(G)\right)
$$


where $\mathcal{B}_{l}^{\sigma}\left(L^{\infty}(G)\right)$ is the space of (bounded) weak* continuous maps on $L^{\infty}(G)$ commuting with left translations.

For abelian locally compact groups $G$, Størmer showed in $[71, \S 4]$ that there exists an isometric homomorphism $\Theta^{l}$ from $M(G)$ into $\mathcal{B}_{\mathcal{R}(G)}^{\sigma}\left(\mathcal{B}\left(L^{2}(G)\right)\right)$, the space of normal bounded $\mathcal{R}(G)$-bimodule morphisms on $\mathcal{B}\left(L^{2}(G)\right)$, which is given by

$$
\left\langle\Theta^{l}(\mu)(x), \rho\right\rangle=\int_{G}\left\langle\lambda(s)^{*} x \lambda(s), \rho\right\rangle d \mu(s)
$$

for $\mu \in M(G), x \in \mathcal{B}\left(L^{2}(G)\right), \rho \in \mathcal{T}\left(L^{2}(G)\right)$. Recall that a bounded map $\Phi$ on $\mathcal{B}\left(L^{2}(G)\right)$ is an $\mathcal{R}(G)$-bimodule if $\Phi(a T b)=a \Phi(T) b$ for all $T \in \mathcal{B}\left(L^{2}(G)\right), a, b \in$ $\mathcal{R}(G)$. This result was extended to nonabelian groups by Ghahramani [26] and further studied by Neufang in his Ph.D. thesis [57]. Neufang showed that $\Theta^{l}$ is actually a completely isometric algebra isomorphism from $M(G)$ onto $\mathcal{C B}_{\mathcal{R}(G)}^{\sigma, L^{\infty}(G)}\left(\mathcal{B}\left(L^{2}(G)\right)\right)$, the space of normal completely bounded $\mathcal{R}(G)$-bimodule morphisms on $\mathcal{B}\left(L^{2}(G)\right)$ which map $L^{\infty}(G)$ into $L^{\infty}(G)$. The global invariance of $L^{\infty}(G)$ under $\Theta^{l}(\mu)$ is due to the relation $\Theta^{l}(\mu)\left(M_{f}\right)=M_{\mu \cdot f}$, where

$$
\mu \cdot f(s)=\int_{G} f(t s) d \mu(t), \quad f \in L^{\infty}(G), \quad s \in G
$$

Not surprisingly, there is a corresponding completely isometric algebra isomorphism

$$
\Theta^{r}: M(G) \cong \mathcal{C B}_{\mathcal{L}(G)}^{\sigma, L^{\infty}(G)}\left(\mathcal{B}\left(L^{2}(G)\right)\right)
$$


In the dual setting, one has the Fourier algebra and their completely bounded multipliers $M_{c b} A(G)$, where a function $\varphi: G \rightarrow \mathbb{C}$ lies in $M_{c b} A(G)$ if $m_{\varphi}(\psi)=\varphi \psi \in$ $A(G)$ for all $\psi \in A(G)$ and $\left\|m_{\varphi}\right\|_{c b}<\infty$. Note that any $\varphi \in M_{c b} A(G)$ is automatically bounded and continuous. Given a completely bounded multiplier $\varphi$, the adjoint

$$
M_{\varphi}=\left(m_{\varphi}\right)^{*}: \mathcal{L}(G) \ni \lambda(s) \mapsto \varphi(s) \lambda(s) \in \mathcal{L}(G)
$$

is a normal completely bounded map on $\mathcal{L}(G)$. It follows that we may identify $M_{c b} A(G)$ completely isometrically with $\mathcal{C B}_{A(G)}^{\sigma}(\mathcal{L}(G))$, the algebra of normal completely bounded maps on $\mathcal{L}(G)$ commuting with the bimodule action of $A(G)$ on $\mathcal{L}(G)$, where $\psi \cdot \lambda(s)=\lambda(s) \cdot \psi=\psi(s) \lambda(s)$ for $\psi \in A(G), s \in G$ [68, Proposition 4.1].

Using a representation theorem of Gilbert [27], which implies that for every $\varphi \epsilon$ $M_{c b} A(G)$ there exists a Hilbert space $H$ and two bounded continuous functions $\xi, \eta$ : $G \rightarrow H$ such that

$$
\varphi\left(s^{-1} t\right)=\langle\eta(t), \xi(s)\rangle
$$

for all $s, t \in G$, Neufang, Ruan and Spronk [58] established the dual version of (4.1) by exhibiting a completely isometric algebra isomorphism

$$
\hat{\Theta}: M_{c b} A(G) \cong \mathcal{C B}_{L^{\infty}(G)}^{\sigma, \mathcal{L}(G)}\left(\mathcal{B}\left(L^{2}(G)\right)\right)
$$

from the completely bounded Fourier multiplier algebra onto the algebra of normal completely bounded $L^{\infty}(G)$-bimodule maps on $\mathcal{B}\left(L^{2}(G)\right)$ which map $\mathcal{L}(G)$ into $\mathcal{L}(G)$. 
If $\varphi \in M_{c b} A(G)$ with Gilbert representation $\xi, \eta: G \rightarrow H$, and $\left(e_{\imath}\right)_{\imath \in I}$ is an orthonormal basis for $H$, the functions $\xi_{\imath}(s)=\left\langle e_{\imath}, \xi(s)\right\rangle$ and $\eta_{\imath}(t)=\left\langle\eta(t), e_{\imath}\right\rangle$ belong to $L^{\infty}(G)$ and the map $\hat{\Theta}(\varphi)$ is given by

$$
\hat{\Theta}(\varphi)(x)=w^{*}-\sum_{\imath \in I} M_{\xi_{2}} x M_{\eta_{\imath}}, \quad x \in \mathcal{B}\left(L^{2}(G)\right)
$$

Here, the invariance of $\mathcal{L}(G)$ under $\hat{\Theta}(\varphi)$ follows from the relation $\hat{\Theta}(\varphi)(\lambda(s))=$ $\varphi(s) \lambda(s), s \in G$.

Since Wendel's work shows $M(G) \cong M_{c b}\left(L^{1}(G)\right)$, we may regard $M_{c b} A(G)$ as the natural dual object of the measure algebra $M(G)$, and equations (4.1) and (4.2) show that both algebras can be represented on the same space $\mathcal{B}\left(L^{2}(G)\right)$ in a manner which perfectly displays their duality.

Shortly afterwards, Junge, Neufang and Ruan [41] unified and generalized the above representation theorems to the setting of locally compact quantum groups. In doing so, they defined a completely bounded (right) multiplier algebra $M_{c b}^{r}\left(L^{1}(\mathbb{G})\right.$ ) of $L^{1}(\mathbb{G})$ for an arbitrary locally compact quantum group $\mathbb{G}$ and showed that there exists a completely isometric algebra isomorphism

$$
\Theta^{r}: M_{c b}^{r}\left(L^{1}(\mathbb{G})\right) \cong \mathcal{C B}_{L^{\infty}(\widehat{\mathbb{G}})}^{\sigma, L^{\infty}(\mathbb{G})}\left(\mathcal{B}\left(L^{2}(\mathbb{G})\right)\right)
$$

In what follows, we give a detailed exposition of the representation theorem (4.3) and how it may be used to generate an intriguing class of quantum channels for every 
locally compact quantum group. Proofs that are not presented may be found in [41].

\subsection{The Representation Theorem}

Contrary to [41], we shall work with left as opposed to right multipliers.

Definition 4.2.1. Let $\mathbb{G}$ be a locally compact quantum group. An element $\hat{b} \in$ $L^{\infty}(\hat{\mathbb{G}})$ is said to be a completely bounded left multiplier of $L^{1}(\mathbb{G})$ if $\hat{b} \lambda(f) \in \lambda\left(L^{1}(\mathbb{G})\right)$ for all $f \in L^{1}(\mathbb{G})$ and the induced map

$$
m_{\hat{b}}^{l}: L^{1}(\mathbb{G}) \ni f \mapsto \lambda^{-1}(\hat{b} \lambda(f)) \in L^{1}(\mathbb{G})
$$

is completely bounded on $L^{1}(\mathbb{G})$. We let $M_{c b}^{l}\left(L^{1}(\mathbb{G})\right)$ denote the space of all completely bounded left multipliers of $L^{1}(\mathbb{G})$. It is easy to see that $M_{c b}^{l}\left(L^{1}(\mathbb{G})\right)$ is a completely contractive Banach algebra with respect to the norm

$$
\left\|\left[\hat{b}_{\imath \jmath}\right]\right\|_{M_{n}\left(M_{c b}^{l}\left(L^{1}(\mathbb{G})\right)\right)}=\left\|\left[m_{\hat{b}_{\imath \jmath}}^{l}\right]\right\|_{c b} .
$$

Recall that the multiplication in $L^{1}(\mathbb{G})$ is given by

$$
\star=\Gamma_{*}: L^{1}(\mathbb{G}) \widehat{\otimes} L^{1}(\mathbb{G}) \ni f \otimes g \mapsto f \star g=\Gamma_{*}(f \otimes g) \in L^{1}(\mathbb{G}) .
$$

A completely bounded map $T$ on $L^{1}(\mathbb{G})$ is said to be a completely bounded left 
centralizer on $L^{1}(\mathbb{G})$ if it satisfies

$$
T(f \star g)=T(f) \star g
$$

for all $f, g \in L^{1}(\mathbb{G})$. We let $C_{c b}^{l}\left(L^{1}(\mathbb{G})\right)$ denote the space of all completely bounded left centralizers of $L^{1}(\mathbb{G})$.

Proposition 4.2.2. [41, Proposition 4.1] If $\hat{b} \in M_{c b}^{l}\left(L^{1}(\mathbb{G})\right)$, then there exists a complete contraction $\tilde{m}_{\hat{b}}^{l}$ on $M(\mathbb{G})$ such that

$$
\tilde{m}_{\hat{b}}^{l}(\mu \star f)=\tilde{m}_{\hat{b}}^{l}(\mu) \star f
$$

for all $\mu \in M(\mathbb{G})$ and $f \in L^{1}(\mathbb{G})$, and $\left.\left(\tilde{m}_{\hat{b}}^{l}\right)\right|_{L^{1}(\mathbb{G})}=m_{\hat{b}}^{l}$. Consequently, $m_{\hat{b}}^{l} \in$ $C_{c b}^{l}\left(L^{1}(\mathbb{G})\right)$.

We therefore obtain the canonical linear injection

$$
m^{l}: M_{c b}^{l}\left(L^{1}(\mathbb{G})\right) \ni \hat{b} \mapsto m_{\hat{b}}^{l} \in C_{c b}^{l}\left(L^{1}(\mathbb{G})\right)
$$

which is a homomorphism since

$$
m_{\hat{a} \hat{b}}^{l}(f)=\lambda^{-1}(\hat{a} \hat{b} \lambda(f))=\lambda^{-1}\left(\hat{a} \lambda\left(m_{\hat{b}}^{l}(f)\right)\right)=m_{\hat{a}}^{l} \circ m_{\hat{b}}^{l}(f) .
$$

for all $\hat{a}, \hat{b} \in M_{c b}^{l}\left(L^{1}(\mathbb{G})\right)$. If $\hat{b}=\lambda(f)$ for some $f \in L^{1}(\mathbb{G})$, then one easily sees that $m_{\hat{b}}^{l}$ is simply left convolution by $f$. 
Taking the adjoint $\Phi_{\hat{b}}^{l}=\left(m_{\hat{b}}^{l}\right)^{*}$, we obtain an injective anti-homomorphism

$$
\Phi^{l}: M_{c b}^{l}\left(L^{1}(\mathbb{G})\right) \ni \hat{b} \mapsto \Phi_{\hat{b}}^{l} \in \mathcal{C B}^{\sigma}\left(L^{\infty}(\mathbb{G})\right)
$$

A normal completely bounded map $\Phi$ on $L^{\infty}(\mathbb{G})$ is said to be left covariant if it satisfies

$$
(\Phi \otimes \iota) \circ \Gamma=\Gamma \circ \Phi
$$

We let $\mathcal{C B}_{\text {cov }}^{\sigma}\left(L^{\infty}(\mathbb{G})\right)$ denote the space of all normal completely bounded left covariant maps on $L^{\infty}(\mathbb{G})$. We see that left covariance is a dual characterization of left centralizers since $T \in C_{c b}^{l}\left(L^{1}(\mathbb{G})\right)$ implies

$$
\left\langle\left(T^{*} \otimes \iota\right) \circ \Gamma(x), f \otimes g\right\rangle=\langle x, T(f) \star g\rangle \quad \text { and } \quad\left\langle\Gamma \circ T^{*}(x), f \otimes g\right\rangle=\langle x, T(f \star g)\rangle
$$

for all $x \in L^{\infty}(\mathbb{G})$ and $f, g \in L^{1}(\mathbb{G})$.

Proposition 4.2.3. [41, Proposition 4.2] If we define the operator space matrix norm

$$
\left\|\left[\hat{b}_{\imath \jmath}\right]\right\|_{M_{n}\left(M_{c b}^{l}\left(L^{1}(\mathbb{G})\right)\right)}=\left\|\left[m_{\hat{b}_{2 \jmath}}^{l}\right]\right\|_{c b}=\left\|\left[\Phi_{\hat{b}_{\imath \jmath}}^{l}\right]\right\|_{c b}
$$

on $M_{c b}^{l}\left(L^{1}(\mathbb{G})\right)$, then

$$
m^{l}: M_{c b}^{l}\left(L^{1}(\mathbb{G})\right) \ni \hat{b} \mapsto m_{\hat{b}}^{l} \in C_{c b}^{l}\left(L^{1}(\mathbb{G})\right)
$$


is a completely isometric homomorphism, and

$$
\Phi^{l}: M_{c b}^{l}\left(L^{1}(\mathbb{G})\right) \ni \hat{b} \mapsto \Phi_{\hat{b}}^{l} \in \mathcal{C B}_{\operatorname{cov}}\left(L^{\infty}(\mathbb{G})\right)
$$

is a completely isometric anti-homomorphism.

In [41, Corollary 4.4], Junge, Neufang and Ruan show that the above maps are actually surjective. Hence, we may regard the newly defined algebra $M_{c b}^{l}\left(L^{1}(\mathbb{G})\right)$ as a concrete realization of $C_{c b}^{l}\left(L^{1}(\mathbb{G})\right)$ or $\mathcal{C B}_{c o v}^{\sigma}\left(L^{\infty}(\mathbb{G})\right)$ inside the von Neumann algebra $L^{\infty}(\hat{\mathbb{G}})$. In what follows we shall identify $M_{c b}^{l}\left(L^{1}(\mathbb{G})\right)$ and $C_{c b}^{l}\left(L^{1}(\mathbb{G})\right)$ without comment. The authors also show that for an element $\mu \in M_{c b}^{l}\left(L^{1}(\mathbb{G})\right)$, the dual $\Phi_{\mu}^{l} \in \mathcal{C B}_{c o v}^{\sigma}\left(L^{\infty}(\mathbb{G})\right)$ may be uniquely extended to a normal completely bounded map $\Theta^{l}(\mu): \mathcal{B}\left(L^{2}(\mathbb{G})\right) \rightarrow \mathcal{B}\left(L^{2}(\mathbb{G})\right)$ in such a way that

$$
1 \otimes \Theta^{l}(\mu)(x)=W\left[\left(\Phi_{\mu}^{l} \otimes \iota\right)\left(W^{*}(1 \otimes x) W\right)\right] W^{*}
$$

for all $x \in \mathcal{B}\left(L^{2}(\mathbb{G})\right)$. We finally obtain the representation theorem.

Theorem 4.2.4. [41, Theorem 4.10] Let $\mathbb{G}$ be a locally compact quantum group. There exists a completely isometric anti-isomorphism $\Theta^{l}$ from $M_{c b}^{l}\left(L^{1}(\mathbb{G})\right)$ onto $\mathcal{C B}_{L^{\infty}\left(\hat{\mathbb{G}}^{\prime}\right)}^{\sigma, L^{\infty}(\mathbb{B})}\left(\mathcal{B}\left(L^{2}(\mathbb{G})\right)\right)$, the algebra of normal completely bounded $L^{\infty}\left(\hat{\mathbb{G}}^{\prime}\right)$-bimodule morphisms which map $L^{\infty}(\mathbb{G})$ into $L^{\infty}(\mathbb{G})$.

Not surprisingly, there is an analoguous representation theorem for right multipliers. We call an element $\hat{b}^{\prime} \in L^{\infty}\left(\hat{\mathbb{G}}^{\prime}\right)$ a completely bounded right multiplier of $L^{1}(\mathbb{G})$ 
if $\rho\left(L^{1}(\mathbb{G})\right) \hat{b}^{\prime} \subseteq \rho\left(L^{1}(\mathbb{G})\right)$, and the induced map

$$
m_{\hat{b}^{\prime}}^{r}: L^{1}(\mathbb{G}) \ni f \mapsto \rho^{-1}\left(\rho(f) \hat{b}^{\prime}\right) \in L^{1}(\mathbb{G})
$$

is completely bounded. Using the same methods, one may obtain the following result.

Theorem 4.2.5. [41, Theorem 4.5] Let $\mathbb{G}$ be a locally compact quantum group. There is a completely isometric isomorphism $\Theta^{r}$ from $M_{c b}^{r}\left(L^{1}(\mathbb{G})\right)$ onto $\mathcal{C B}_{L^{\infty}(\hat{\mathbb{G}})}^{\sigma, L^{\infty}(\mathbb{G})}\left(\mathcal{B}\left(L^{2}(\mathbb{G})\right)\right)$, the algebra of normal completely bounded $L^{\infty}(\hat{\mathbb{G}})$-bimodule morphisms which map $L^{\infty}(\mathbb{G})$ into $L^{\infty}(\mathbb{G})$.

In the case of commutative quantum groups, we have $M_{c b}^{l}\left(L^{1}\left(\mathbb{G}_{a}\right)\right)=M_{c b}^{l}\left(L^{1}(G)\right) \cong$ $M(G)$ for a locally compact group $G$, and we recover the (left) representation $\Theta^{l}$ in (4.1). For co-commutative quantum groups, $M_{c b}^{l}\left(L^{1}\left(\mathbb{G}_{s}\right)\right)=M_{c b}^{r}\left(L^{1}\left(\mathbb{G}_{s}\right)\right)=M_{c b} A(G)$, the completely bounded Fourier multiplier algebra, and we obtain the representation $\hat{\Theta}$ in $(4.2)$.

When $\mathbb{G}$ is discrete, i.e., when $L^{1}(\mathbb{G})$ is unital, every multiplier $\mu \in M_{c b}^{l}\left(L^{1}(\mathbb{G})\right)$ is of the form $\lambda(f)$ for some $f \in L^{1}(\mathbb{G})$. In this case it follows that

$$
\Theta^{l}(\lambda(f))(x)=\langle f \otimes \iota, \tilde{\Gamma}(x)\rangle=\left\langle f \otimes \iota, W^{*}(1 \otimes x) W\right\rangle
$$

for all $x \in \mathcal{B}\left(L^{2}(\mathbb{G})\right)$. 


\subsection{Positive Definite Functionals}

A fundamental object in abstract harmonic analysis is the class of positive definite functions. They were crucial in the development of Fourier analysis on locally compact abelian groups, and their connection with unitary representations led to the celebrated Gelfand-Raikov theorem [25, Thoerem 3.34].

We begin this section with a review of the relevant classical theory. We then define the notion of positive definite functionals on locally compact quantum groups and pursue certain generalizations of classical results such as the connection with unitary co-representations. Finally, we discuss completely positive multipliers which will be the main focus of the thesis thereafter.

\subsubsection{Classical Theory}

Throughout this entire section, $G$ will denote a locally compact group. Details may be found in $[25, \S 3.3]$.

Definition 4.3.1. A function $\varphi: G \rightarrow \mathbb{C}$ is positıve definute if

$$
\sum_{\imath, \jmath=1}^{n} \alpha_{\imath} \overline{\alpha_{\jmath}} \varphi\left(s_{\jmath}^{-1} s_{\imath}\right) \geq 0
$$

for all $\alpha_{1}, \ldots, \alpha_{n} \in \mathbb{C}, s_{1}, \ldots, s_{n} \in G$ and $n \in \mathbb{N}$.

Note that, by taking $n=2, s_{1}=s$ and $s_{2}=e$, this condition implies that the 
matrix

$$
\left(\begin{array}{cc}
\varphi(e) & \varphi(s) \\
\varphi\left(s^{-1}\right) & \varphi(e)
\end{array}\right)
$$

is positive semi-definite. Hence, $\varphi\left(s^{-1}\right)=\overline{\varphi(s)}$ and $\varphi(e)^{2}-\varphi(s) \varphi\left(s^{-1}\right) \geq 0$ so that $|\varphi(s)| \leq \varphi(e)$ for all $s \in G$. In particular, positive definite functions are bounded.

When a function $\varphi: G \rightarrow \mathbb{C}$ is bounded and continuous, it follows that $\varphi$ is positive definite if and only if it defines a positive linear functional on $L^{1}(G)[25$, Proposition 3.35], that is

$$
\left\langle\varphi, f^{*} * f\right\rangle=\int_{G} \varphi(s) f^{*} * f(s) d s \geq 0, \quad f \in L^{1}(G) .
$$

The set of continuous positive definite functions will be denoted $\mathcal{P}(G)$, and those of norm one $\mathcal{P}_{1}(G)$. Observe that $\varphi \in \mathcal{P}(G)$ satisfies $\|\varphi\|_{\infty}=\varphi(e)$.

Definition 4.3.2. A unitary representation of $G$ is a homomorphism $\pi$ from $G$ into $\mathcal{U}\left(H_{\pi}\right)$, the group of unitary operators on some nonzero Hilbert space $H_{\pi}$, that is continuous with respect to the strong operator topology.

We shall use the same terminology for unitary representations as we did for *representations of $C^{*}$-algebras in $\S 1.2$. Notably, two unitary representations $\pi_{1}$ and $\pi_{2}$ are said to be unitarily equivalent if there exists a unitary $U: H_{\pi_{1}} \rightarrow H_{\pi_{2}}$ such that $\pi_{2}(s)=U \pi_{1}(s) U^{*}$ for all $s \in G$.

Any unitary representation of $G$ determines a spatially nondegenerate *-representation of the group algebra $L^{1}(G)$ by the following recipe. If $f \in L^{1}(G)$, 
then $\pi(f)$ is the bounded linear operator on $H_{\pi}$ given by

$$
\langle\pi(f), \rho\rangle=\int_{G} f(s)\langle\pi(s), \rho\rangle d s, \quad \rho \in \mathcal{T}\left(H_{\pi}\right)
$$

Similarly to the setting of $C^{*}$-algebras, there is an intimate connection between positive definite functions and unitary representations.

Proposition 4.3.3. [25, Proposition 3.15] If $\pi$ is a unitary representation of $G$ and $\xi \in H_{\pi}$, then the function $\varphi(s)=\langle\pi(s) \xi$, $\xi\rangle$ lies in $\mathcal{P}(G)$.

Since $\varphi \in \mathcal{P}(G)$ defines a positive linear functional on the involutive Banach algebra $L^{1}(G)$, one may apply the GNS construction to achieve the converse of the above proposition.

Theorem 4.3.4. [25, Theorem 3.20] Given $\varphi \in \mathcal{P}(G)$, there exists a cyclic unitary representation $\left(\pi_{\varphi}, \xi_{\varphi}\right)$ such that $\varphi=\left\langle\pi_{\varphi}(\cdot) \xi_{\varphi}, \xi_{\varphi}\right\rangle \in L^{\infty}(G)$. Moreover, $\left(\pi_{\varphi}, \xi_{\varphi}\right)$ is unique up to unitary equivalence.

The set $\mathcal{P}(G)$ is a convex cone inside $L^{\infty}(G)$ containing $\mathcal{P}_{1}(G)=\{\varphi \in \mathcal{P}(G)$ : $\varphi(e)=1\}$ as a convex subset. Denoting $\mathcal{E}\left(\mathcal{P}_{1}(G)\right)$ the extreme points of $\mathcal{P}_{1}(G)$, the following result provides a similar interpretation of $\mathcal{E}\left(\mathcal{P}_{1}(G)\right)$ to Theorem 2.2.11.

Theorem 4.3.5. [25, Theorem 3.25] If $\varphi \in \mathcal{P}_{1}(G)$, then $\varphi \in \mathcal{E}\left(\mathcal{P}_{1}(G)\right)$ if and only if the unitary representation $\pi_{\varphi}$ is irreducible.

In connection with the representation of $M_{c b} A(G)$, it was shown that $\varphi \in M_{c b} A(G)$ generates a completely positive map $\hat{\Theta}(\varphi) \in \mathcal{C B}_{L^{\infty}(G)}^{\sigma, \mathcal{L}(G)}\left(\mathcal{B}\left(L^{2}(G)\right)\right)$ if and only if $\varphi \in$ 
$\mathcal{P}(G)[58$, Lemma 4.1]. In order to investigate the generalization of this result to the quantum setting, we must adopt yet another characterization of positive definite functions.

Proposition 4.3.6. [25, $\S 7.1] \mathcal{P}(G)$ us ısometrically ısomorphic to the set of positvve linear functionals on $C^{*}(G)$, the full group $C^{*}$-algebra of $G$.

\subsubsection{Quantum Theory}

Throughout this section, unless specified otherwise, $\mathbb{G}$ denotes a fixed locally compact quantum group. We will denote by $\otimes$, the minimal tensor product of $C^{*}$-algebras.

Definition 4.3.7. A posttve defintte functıonal on $\mathbb{G}$ is a positive element in $C_{u}(\mathbb{G})^{*}$. The set of positive definite functionals is denoted $\mathcal{P}(\mathbb{G})$, and we denote the set of states in $C_{u}(\mathbb{G})^{*}$ by $\mathcal{P}_{1}(\mathbb{G})$.

Recall from chapter 2 that $C_{u}\left(\mathbb{G}_{a}\right)=C_{0}(G)$, so in the commutative case $\mathcal{P}\left(\mathbb{G}_{a}\right)$ is the set of positive measures in $M(G), \mathcal{P}_{1}\left(\mathbb{G}_{a}\right)$ being the probability measures. On the other hand, $C_{u}\left(\mathbb{G}_{s}\right)=C^{*}(G)$, so we recover the usual positive definite functions in the co-commutative case. For this class we established a correspondence between elements of $\mathcal{P}(G)$ and unitary representations of $G$.

In the general quantum group setting, one passes from unitary representations to unitary co-representations. For instance, the fundamental unitary $W_{a}$ may be viewed as the "left regular co-representation" of $\mathbb{G}_{a}$ as it is intimately related to the usual left regular representation of a locally compact group. The prefix "co" is justified by 
the following identities

$$
\left(\Gamma_{a} \otimes \iota\right)\left(W_{a}\right)=\left(W_{a}\right)_{13}\left(W_{a}\right)_{23} \quad \text { and } \quad\left(\iota \otimes \hat{\Gamma_{a}}\right)\left(W_{a}\right)=\left(W_{a}\right)_{13}\left(W_{a}\right)_{12}
$$

Motivated by this, we seek a generalized relationship between positive definite functionals and unitary co-representations.

Definition 4.3.8. If $\mathcal{A} \subseteq \mathcal{B}(H)$ is a $C^{*}$-algebra, a unitary co-representation of $\hat{\mathbb{G}}$ is a unitary element $U \in M\left(\mathcal{A} \otimes C_{0}(\hat{\mathbb{G}})\right)$ satisfying $(\iota \otimes \hat{\Gamma})(U)=U_{13} U_{12}$. Two unitary co-representations $U_{1}$ and $U_{2}$ are unitarily equivalent if there exists a unitary $\mathcal{U}: H_{1} \rightarrow H_{2}$ such that $(\mathcal{U} \otimes \iota) U_{1}=U_{2}(\mathcal{U} \otimes \iota)$.

One of the major results of Kustermans in [48] is the existence of a "universal" co-representation $\mathcal{V} \in M\left(C_{u}(\mathbb{G}) \otimes C_{0}(\hat{\mathbb{G}})\right)$ satisfying

1. $\hat{\lambda}_{u}(\hat{f})=(\iota \otimes \hat{f})\left(\mathcal{V}^{*}\right)$ for all $\hat{f} \in L_{*}^{1}(\hat{\mathbb{G}})$,

2. $(\iota \otimes \pi) \circ \Gamma_{u}(x)=\mathcal{V}^{*}(1 \otimes \pi(x)) \mathcal{V}$ for all $x \in C_{u}(\mathbb{G})$,

where $\hat{\lambda}_{u}$ is the embedding of $L_{*}^{1}(\hat{\mathbb{G}})$ into $C_{u}(\mathbb{G})$, and $\pi: C_{u}(\mathbb{G}) \rightarrow C_{0}(\mathbb{G})$ is the (unique) extension of $\hat{\lambda}: L_{*}^{1}(\hat{\mathbb{G}}) \rightarrow C_{0}(\mathbb{G})$ [48, Proposition 5.1]. Moreover,

$$
C_{u}(\mathbb{G})=\overline{\operatorname{span}\left\{(\iota \otimes \hat{f})(\mathcal{V}): \hat{f} \in L^{1}(\hat{\mathbb{G}})\right\}} \|^{\|} .
$$


Proposition 4.3.9. [48, Proposition 5.3] Let $\mathcal{A}$ be a $C^{*}$-algebra and $U \in M(\mathcal{A} \otimes$ $\left.C_{0}(\hat{\mathbb{G}})\right)$ be a unitary co-representation of $\hat{\mathbb{G}}$. Then there exists a unique nondegenerate *-homomorphism $\pi_{U}: C_{u}(\mathbb{G}) \rightarrow M(\mathcal{A})$ such that $\left(\pi_{U} \otimes \iota\right)(\mathcal{V})=U$.

With the notation of Proposition 4.3.9, if $\mathcal{A} \subseteq \mathcal{B}(H)$ for a Hilbert space $H$, taking any vector $\xi \in H$ yields $\mu:=\omega_{\xi} \circ \pi_{U} \in \mathcal{P}(\mathbb{G})$. Furthermore, $(\mu \otimes \iota)(\mathcal{V})=\left(\omega_{\xi} \otimes \iota\right)(U)$.

Proposition 4.3.10. Let $\mu \in \mathcal{P}(\mathbb{G})$ with cyclic GNS representation $\left(\pi_{\mu}, \xi\right)$. Then there exists a $C^{*}$-algebra $\mathcal{A}$, and a unique unitary co-representation $U_{\mu} \in M(\mathcal{A} \otimes$ $\left.C_{0}(\hat{\mathbb{G}})\right)$ of $\hat{\mathbb{G}}$ (up to unitary equivalence) such that $(\mu \otimes \iota)(\mathcal{V})=\left(\omega_{\xi} \otimes \iota\right)\left(U_{\mu}\right)$.

Proof. As $\pi_{\mu}: C_{u}(\mathbb{G}) \rightarrow \mathcal{B}\left(H_{\mu}\right)$ is a $*$-homomorphism, its image $\mathcal{A}:=\pi_{\mu}\left(C_{u}(\mathbb{G})\right)$ is a $C^{*}$-algebra [62, Proposition 2.4.9]. Let $\left(e_{\alpha}\right)_{\alpha \in A}$ be a bounded approximate identity in $C_{u}(\mathbb{G})$. Viewing $\pi_{\mu}: C_{u}(\mathbb{G}) \rightarrow M(\mathcal{A})$, it follows by norm continuity of $\pi_{\mu}$ that for every $x \in C_{u}(\mathbb{G}), \pi_{\mu}\left(x e_{\alpha}\right) \rightarrow \pi_{\mu}(x)$ in $\mathcal{A}$. Hence,

$$
\left\{\pi_{\mu}(x) \square a \mid x \in C_{u}(\mathbb{G}), a \in \mathcal{A}\right\}=\left\{\pi_{\mu}(x y) \mid x, y \in C_{u}(\mathbb{G})\right\}
$$

is norm dense in $\mathcal{A}$, making $\pi_{\mu}$ nondegenerate. Taking $U_{\mu}=\left(\pi_{\mu} \otimes \iota\right)(\mathcal{V})$ yields our desired unitary co-representation. Note that if $\left(\pi_{\mu}^{\prime}, \xi^{\prime}\right)$ is another cyclic GNS construction for $\mu$, then the associated unitary co-representation $U_{\mu}^{\prime}$ is unitarily equivalent to $U_{\mu}$.

We have thus achieved the generalized relationship between positive definite functionals and unitary co-representations. To see the connection with completely positive 
multipliers, first note that the identification $C_{c b}^{l}\left(L^{1}(\mathbb{G})\right) \cong \mathcal{C B}_{\text {cov }}^{\sigma}\left(L^{\infty}(\mathbb{G})\right)$ induces a natural ordering on $C_{c b}^{l}\left(L^{1}(\mathbb{G})\right)$ given by

$$
C_{c b}^{l}\left(L^{1}(\mathbb{G})\right)^{+}=\left\{\mu \in C_{c b}^{l}\left(L^{1}(\mathbb{G})\right): \Phi_{\mu}^{l} \in \mathcal{C B}_{c o v}^{\sigma}\left(L^{\infty}(\mathbb{G})\right) \text { is completely positive }\right\}
$$

Under the usual ordering of $\mathcal{C B}^{\sigma}\left(\mathcal{B}\left(L^{2}(\mathbb{G})\right)\right)$ by completely positive maps, it follows that $\Theta^{l}: C_{c b}^{l}\left(L^{1}(\mathbb{G})\right) \rightarrow \mathcal{C} \mathcal{B}_{L^{\infty}\left(\hat{\mathbb{G}}^{\prime}\right)}^{\left.\sigma, L^{\infty}\right)}\left(\mathcal{B}\left(L^{2}(\mathbb{G})\right)\right)$ is order preserving by equation (4.4).

Now, it is known that $C_{u}(\mathbb{G})^{*}$ always embeds into $C_{c b}^{l}\left(L^{1}(\mathbb{G})\right)[18$, Proposition $8.3]$, and that for both the commutative, and co-commutative cases we have $[58, \S 3,4]$

$$
C_{c b}^{l}\left(L^{1}(\mathbb{G})\right)^{+}=\mathcal{P}(\mathbb{G})
$$

It has also recently been announced in a preprint that equality (4.7) holds for general locally compact quantum groups [19]. However, using unitary co-representations, we can explicitly see one direction, yielding a concrete method to generate new quantum channels.

Let $i: L^{1}(\mathbb{G}) \rightarrow C_{u}(\mathbb{G})^{*}$ be the canonical embedding (see $\S 3.5$ ), and recall that the lifting $\pi: C_{u}(\mathbb{G}) \rightarrow C_{0}(\mathbb{G})$ of $\hat{\lambda}: L_{*}^{1}(\hat{\mathbb{G}}) \rightarrow C_{0}(\mathbb{G})$ satisfies $\left.\pi^{*}\right|_{L^{1}(\mathbb{G})}=i$. Then the map

$$
m_{\mu}^{l}(f)=i^{-1}\left(\mu \star_{u} i(f)\right), \quad \mu \in C_{u}(\mathbb{G})^{*}, \quad f \in L^{1}(\mathbb{G})
$$

defines an element of $C_{c b}^{l}\left(L^{1}(\mathbb{G})\right)$. Its adjoint in $\mathcal{C B}_{\text {cov }}^{\sigma}\left(L^{\infty}(\mathbb{G})\right)$ will be denoted $\Phi_{\mu}$. 
Theorem 4.3.11. If $\mu \in \mathcal{P}(\mathbb{G})$, then $\Phi_{\mu}$ is completely positive. If in addition $\mu \in$ $\mathcal{P}_{1}(\mathbb{G})$, then $\Phi_{\mu}$ is unital.

Proof. We follow along similar lines as Daws in [17, Theorem 6.1]. Let $\left(\pi_{\mu}, \xi\right)$ be a GNS representation associated with $\mu$, and let $x=\hat{\lambda}(\hat{f}) \in C_{0}(\mathbb{G})$ for some $\hat{f} \in L_{*}^{1}(\hat{\mathbb{G}})$. Then, for all $f \in L^{1}(\mathbb{G})$,

$$
\begin{aligned}
\left\langle\Phi_{\mu}(x), f\right\rangle & =\left\langle x, m_{\mu}^{l}(f)\right\rangle=\left\langle\hat{\lambda}(\hat{f}), i^{-1}\left(\mu \star_{u} i(f)\right)\right\rangle \\
& =\left\langle\pi \circ \hat{\lambda}_{u}(\hat{f}), i^{-1}\left(\mu \star_{u} i(f)\right)\right\rangle=\left\langle\hat{\lambda}_{u}(\hat{f}), \mu \star_{u} i(f)\right\rangle \\
& =\left\langle\Gamma_{u}\left(\hat{\lambda}_{u}(\hat{f})\right), \mu \otimes i(f)\right\rangle=\left\langle(\iota \otimes \pi)\left(\Gamma_{u}\left(\hat{\lambda}_{u}(\hat{f})\right)\right), \mu \otimes f\right\rangle \\
& =\left\langle\mathcal{V}^{*}\left(1 \otimes \pi\left(\hat{\lambda}_{u}(\hat{f})\right)\right) \mathcal{V}, \mu \otimes f\right\rangle=\left\langle\mathcal{V}^{*}(1 \otimes x) \mathcal{V}, \mu \otimes f\right\rangle \\
& =\left\langle\left(\pi_{\mu} \otimes \iota\right) \mathcal{V}^{*}(1 \otimes x) \mathcal{V}, \omega_{\xi} \otimes f\right\rangle=\left\langle U_{\mu}^{*}(1 \otimes x) U_{\mu}, \omega_{\xi} \otimes f\right\rangle \\
& =\left\langle\left(\omega_{\xi} \otimes \iota\right)\left(U_{\mu}^{*}(1 \otimes x) U_{\mu}\right), f\right\rangle
\end{aligned}
$$

As $L_{*}^{1}(\hat{\mathbb{G}})$ is norm dense in $L^{1}(\hat{\mathbb{G}}), \hat{\lambda}\left(L_{*}^{1}(\hat{\mathbb{G}})\right)$ is norm dense in $C_{0}(\mathbb{G})$, and since $C_{0}(\mathbb{G})$ is weak* dense in $L^{\infty}(\mathbb{G})$, by weak* continuity it follows that

$$
\Phi_{\mu}(x)=\left(\omega_{\xi} \otimes \iota\right)\left(U_{\mu}^{*}(1 \otimes x) U_{\mu}\right), \quad x \in L^{\infty}(\mathbb{G})
$$

It is now clear that $\Phi_{\mu}$ is completely positive as a composition of completely positive maps. If $\mu \in \mathcal{P}_{1}(\mathbb{G})$, then $\|\xi\|=1$, which makes $\Phi_{\mu}$ unital. 
Corollary 4.3.12. If $\mu \in \mathcal{P}_{1}(\mathbb{G})$, then $\Theta^{l}(\mu)$ is a quantum channel on $\mathcal{B}\left(L^{2}(\mathbb{G})\right)$.

Using the relationship between positive definite functionals and unitary co-representations, we may provide an explicit Kraus representation of the quantum channel $\Theta^{l}(\mu)$ for any $\mu \in \mathcal{P}_{1}(\mathbb{G})$.

Theorem 4.3.13. Let $\mu=\left(\pi_{\mu}, \xi\right) \in \mathcal{P}_{1}(\mathbb{G})$ with corresponding unitary co-representation $U_{\mu} \in M\left(\mathcal{A} \otimes C_{0}(\hat{\mathbb{G}})\right)$. Then if $\mathcal{A} \subseteq \mathcal{B}(H)$ for some Hilbert space $H$, and $\left(e_{\imath}\right)_{\imath \in I}$ is an orthonormal basıs for $H$, then

$$
\Theta^{l}(\mu)(x)=w^{*}-\sum_{\imath \in I} W_{\imath}^{*} x W_{\imath}, \quad x \in \mathcal{B}\left(L^{2}(\mathbb{G})\right)
$$

where $W_{\iota}=\left(\omega_{\xi, e_{\imath}} \otimes \iota\right)\left(U_{\mu}\right) \in L^{\infty}(\hat{\mathbb{G}})$.

Proof. If $\theta_{e_{2}, e_{2}}$ denotes the rank-one operator $e_{2} e_{\imath}^{*}, i \in I$, then $w^{*}-\sum_{\imath \in I} \theta_{e_{2}, e_{2}}=$ $1_{H}$. Since multiplication in $\mathcal{B}(H) \bar{\otimes} \mathcal{B}\left(L^{2}(\mathbb{G})\right) \supseteq M\left(\mathcal{A} \otimes C_{0}(\hat{\mathbb{G}})\right)$ is separately weak* continuous, and $\omega_{\xi}$ is a normal state, equation (4.8) gives

$$
\Phi_{\mu}(x)=w^{*}-\sum_{\imath \in I}\left(\omega_{\xi} \otimes \iota\right)\left(U_{\mu}^{*}\left(\theta_{e_{2}, e_{2}} \otimes x\right) U_{\mu}\right)
$$


But if $i \in I, \eta, \zeta \in L^{2}(\mathbb{G})$, we have

$$
\begin{aligned}
\left\langle\left(\omega_{\xi} \otimes \iota\right)\left(U_{\mu}^{*}\left(\theta_{e_{\imath}, e_{\imath}} \otimes x\right) U_{\mu}\right) \eta, \zeta\right\rangle & =\left\langle U_{\mu}^{*}\left(\theta_{e_{\imath}, e_{\imath}} \otimes x\right) U_{\mu}(\xi \otimes \eta), \xi \otimes \zeta\right\rangle \\
& =\left\langle\left(\theta_{e_{\imath}, e_{\imath}} \otimes x\right) U_{\mu}(\xi \otimes \eta), U_{\mu}(\xi \otimes \zeta)\right\rangle \\
& =\left\langle x\left(\omega_{\xi, e_{\imath}} \otimes \iota\right)\left(U_{\mu}\right) \eta,\left(\omega_{\xi, e_{\imath}} \otimes \iota\right)\left(U_{\mu}\right) \zeta\right\rangle \\
& =\left\langle W_{\imath}^{*} x W_{\imath} \eta, \zeta\right\rangle
\end{aligned}
$$

where $W_{\imath}=\left(\omega_{\xi, e_{\imath}} \otimes \iota\right)\left(U_{\mu}\right) \in L^{\infty}(\hat{\mathbb{G}})$. Hence,

$$
\Phi_{\mu}(x)=w^{*}-\sum_{\imath \in I} W_{\imath}^{*} x W_{\imath}
$$

Now, $\Theta^{l}(\mu)$ is an $L^{\infty}\left(\hat{\mathbb{G}}^{\prime}\right)$-bimodule map on $\mathcal{B}\left(L^{2}(\mathbb{G})\right)$, and its restriction to $L^{\infty}(\mathbb{G})$ is $\Phi_{\mu}$, so if $\hat{x}^{\prime} \in L^{\infty}\left(\hat{\mathbb{G}}^{\prime}\right)$ and $x \in L^{\infty}(\mathbb{G})$,

$$
\Theta^{l}(\mu)\left(\hat{x}^{\prime} x\right)=\hat{x}^{\prime} \Phi_{\mu}(x)=\hat{x}^{\prime} \sum_{\imath \in I} W_{\imath}^{*} x W_{\imath}=\sum_{\imath \in I} W_{\imath}^{*} \hat{x}^{\prime} x W_{\imath}
$$

since $W_{\imath} \in L^{\infty}(\hat{\mathbb{G}})$ for every $i \in I$, and multiplication in $\mathcal{B}\left(L^{2}(\mathbb{G})\right)$ is separately weak* continuous. As $\mu \in \mathcal{P}_{1}(\mathbb{G})$, the above calculations imply that the linear map $\Psi(x)=w^{*}-\sum_{\imath \in I} W_{\imath}^{*} x W_{\imath}, x \in \mathcal{B}\left(L^{2}(\mathbb{G})\right)$, is unital and completely positive. Hence, by [61, Proposition 3.6], $\|\Psi\|_{c b}=\|\Psi(1)\|=\left\|w^{*}-\sum_{\imath \in I} W_{\imath}^{*} W_{\imath}\right\|=1$, so $\Psi$ belongs to $\mathcal{C B}^{\sigma}\left(\mathcal{B}\left(L^{2}(\mathbb{G})\right)\right)[28]$. Thus, $\Psi$ is normal, and it agrees with $\Theta^{l}(\mu)$ on the weak* dense 
subspace $\operatorname{span} L^{\infty}\left(\hat{\mathbb{G}}^{\prime}\right) L^{\infty}(\mathbb{G})$ of $\mathcal{B}\left(L^{2}(\mathbb{G})\right)$, so by Theorem 3.3.6 we must have

$$
\Theta^{l}(\mu)(x)=w^{*}-\sum_{\imath \in I} W_{\imath}^{*} x W_{\imath}, \quad x \in \mathcal{B}\left(L^{2}(\mathbb{G})\right)
$$

In [5, Theorem 3.1], it was shown that $\mathbb{G}$ is co-amenable if and only if $M(\mathbb{G}) \cong$ $C_{u}(\mathbb{G})^{*}$, which is equivalent to $C_{0}(\mathbb{G}) \cong C_{u}(\mathbb{G})$. In this case, $M_{c b}^{l}\left(L^{1}(\mathbb{G})\right) \cong M(\mathbb{G})$ [36, Theorem 4.2], $M(\mathbb{G})$ has a positive definite unit $\varepsilon$, and we have equality in (4.7):

Proposition 4.3.14. The following are equivalent for a co-amenable locally compact quantum group $\mathbb{G}$ :

1. $\mu \in \mathcal{P}(\mathbb{G})$;

2. $\Phi_{\mu}$ is completely positive;

3. $\Phi_{\mu}$ is positive.

Consequently, $C_{c b}^{l}\left(L^{1}(\mathbb{G})\right)^{+}=\mathcal{P}(\mathbb{G})$.

Proof. (1) $\Rightarrow(2) \Rightarrow(3)$ is clear. We show (3) $\Rightarrow(1)$. Suppose $\mu \in M(\mathbb{G})$ with $\Phi_{\mu}$ positive. Then $\left.\Phi_{\mu}\right|_{C_{0}(\mathbb{G})}: C_{0}(\mathbb{G}) \rightarrow C_{0}(\mathbb{G})$ is a complete contraction whose adjoint $\tilde{m}_{\mu}^{l}: M(\mathbb{G}) \rightarrow M(\mathbb{G})$ is given by left convolution by [41, Proposition 4.1]. Hence, if $x \in C_{0}(\mathbb{G})^{+}$,

$$
\langle\mu, x\rangle=\langle\mu \star \varepsilon, x\rangle=\left\langle\tilde{m}_{\mu}^{l}(\varepsilon), x\right\rangle=\left\langle\varepsilon, \Phi_{\mu}(x)\right\rangle \geq 0
$$

since $\varepsilon$ is positive definite. Therefore, $\mu \in M(\mathbb{G})^{+}=C_{c b}^{l}\left(L^{1}(\mathbb{G})\right)^{+}=\mathcal{P}(\mathbb{G})$. 


\subsection{Harmonic Operators}

Let $G$ be a locally compact group, and let $\mu$ be a probability measure on $G$, viewed as a completely bounded left centralizer of $L^{1}(G)$. Then the associated covariant map $\Phi_{\mu}$ on $L^{\infty}(G)$ is unital, completely positive and satisfies $\Phi_{\mu}(f)=f * \mu, f \in L^{1}(G)$. The fixed points of this map are called $\mu$-harmonic functions, and form a self-adjoint weak* closed subspace of $L^{\infty}(G)$, denoted $\mathcal{H}_{\mu}$. Remarkably, there is a product $\triangle$ on $\mathcal{H}_{\mu}$, usually different from that in $L^{\infty}(G)$, turning it into an abelian von Neumann algebra.

Over the past decade the notion of "harmonicity" has been studied in several different contexts. In [40], Jaworski and Neufang replaced the action of $\mu$ on $L^{\infty}(G)$ with the extended action of $\Theta^{l}(\mu)$ on $\mathcal{B}\left(L^{2}(G)\right)$, and characterized the corresponding fixed points as a certain crossed product. In [14], Chu and Lau replaced $L^{\infty}(G)$ by $\mathcal{L}(G)$ and studied the canonical action of a positive definite function $\varphi$ on $\mathcal{L}(G)$. In this dual setting, they showed that the fixed point set $\mathcal{H}_{\varphi}$ is always a von Neumann subalgebra of $\mathcal{L}(G)$, in stark contrast to the classical theory. Finally in [59], Neufang and Runde studied the corresponding extended action of $\hat{\Theta}(\varphi)$ on $\mathcal{B}\left(L^{2}(G)\right)$, and described its fixed points, leading to the concept of " $\varphi$-harmonic operators" on $\mathcal{B}\left(L^{2}(G)\right)$

In the quantum group setting, Izumi investigated the fixed points of Markov operators arising from discrete and compact quantum groups, coining the term "noncommutative Poisson boundaries" $[38,39]$. This motivated Kalantar, Neufang and 
Ruan to obtain an explicit description of the noncommutative Poisson boundaries over arbitrary locally compact quantum groups [46]. This section is devoted to an outline of the main results in [46] relevant to our study of quantum channels.

Let $\mathbb{G}$ be a locally compact quantum group, and let $M_{c p}^{1}\left(L^{1}(\mathbb{G})\right)$ be the subset of $M_{c b}^{l}\left(L^{1}(\mathbb{G})\right)$ consisting of those multipliers $\mu$ for which $\Phi_{\mu}$ is unital and completely positive. Assuming the main result of the recent preprint, we may write $M_{c p}^{1}\left(L^{1}(\mathbb{G})\right)=$ $\mathcal{P}_{1}(\mathbb{G})[19]$. For any such $\mu$, we denote the fixed points of $\Phi_{\mu}$ and $\Theta^{l}(\mu)$ by $\mathcal{H}_{\mu}$ and $\tilde{\mathcal{H}}_{\mu}$, respectively. Then $\mathcal{H}_{\mu}$ and $\tilde{\mathcal{H}}_{\mu}$ are weak* closed self-adjoint subspaces of $L^{\infty}(\mathbb{G})$ and $\mathcal{B}\left(L^{2}(\mathbb{G})\right)$, called the $\mu$-harmonic operators of $\Phi_{\mu}$ and $\Theta^{l}(\mu)$. As in the classical case, the Choi-Effros product turns $\mathcal{H}_{\mu}$ and $\tilde{\mathcal{H}}_{\mu}$ into von Neumann algebras. This product is defined as follows.

For $\mu \in \mathcal{P}_{1}(\mathbb{G})$, define a projection of norm one $\mathcal{E}_{\mu}: L^{\infty}(\mathbb{G}) \rightarrow L^{\infty}(\mathbb{G})$ by taking

$$
\mathcal{E}_{\mu}(x)=w^{*}-\lim _{n \mapsto \mathcal{U}} \frac{1}{n} \sum_{k=1}^{n} \Phi_{\mu}^{k}(x)
$$

with respect to a free ultrafilter $\mathcal{U}$ on $\mathbb{N}$. It follows that $\mathcal{E}\left(L^{\infty}(\mathbb{G})\right)=\mathcal{H}_{\mu}$ and that the (associative) product

$$
x \triangle y=\mathcal{E}_{\mu}(x y)
$$

defines a von Neumann algebra structure on $\mathcal{H}_{\mu}$ [12, Theorem 3.1]. Note that this product is independent of the chosen free ultrafilter as every completely positive isometric linear isomorphism between von Neumann algebras is a $*$-isomorphism [38, 
§2.5]. The product on $\tilde{\mathcal{H}}_{\mu}$ is defined similarly. As $\Theta^{l}(\mu)_{L_{L^{\infty}(\mathbb{G})}}=\Phi_{\mu}$, it follows that $\mathcal{H}_{\mu}$ is a von Neumann subalgebra of $\tilde{\mathcal{H}}_{\mu}$. We may also define an action of $\mathbb{G}$ on $\mathcal{H}_{\mu}$.

Definition 4.4.1. Let $N$ be a von Neumann algebra. A normal, unital $*$-homomorphism $\alpha: N \rightarrow N \bar{\otimes} L^{\infty}(\mathbb{G})$ is a right co-action of $\mathbb{G}$ on $N$ if

$$
(\alpha \otimes \iota) \circ \alpha=(\iota \otimes \Gamma) \circ \alpha
$$

The crossed product of $N$ with $\mathbb{G}$, denoted $N \rtimes_{\alpha} \mathbb{G}$, is defined to be the von Neumann subalgebra of $N \bar{\otimes} \mathcal{B}\left(L^{2}(\mathbb{G})\right)$ generated by $\alpha(N)$ and $1 \otimes L^{\infty}\left(\hat{\mathbb{G}}^{\prime}\right)$.

In what follows, $\mathbb{G}$ will denote a fixed locally compact quantum group. For notational simplicity we write $\Gamma_{\mu}$ for $\left.\Gamma\right|_{\mathcal{H}_{\mu}}$, and $\tilde{\Gamma}_{\mu}$ for $\left.\tilde{\Gamma}\right|_{\tilde{\mathcal{H}}_{\mu}}$.

Proposition 4.4.2. [ 46 , Theorem 7.2] If $\mu \in \mathcal{P}_{1}(\mathbb{G})$, then $\Gamma_{\mu}$ defines a right co-action of $\mathbb{G}$ on $\mathcal{H}_{\mu}$.

Proposition 4.4.3. [46, Theorem 7.3] If $\mu \in \mathcal{P}_{1}(\mathbb{G})$, then $\tilde{\Gamma}_{\mu}$ is a normal, injective, unital $*$-homomorphism from $\tilde{\mathcal{H}}_{\mu}$ into $\mathcal{H}_{\mu} \bar{\otimes} \mathcal{B}\left(L^{2}(\mathbb{G})\right)$.

One of the main results of [46] identifies the range of $\tilde{\Gamma}_{\mu}$.

Theorem 4.4.4. [46, Theorem 7.5] If $\mu \in \mathcal{P}_{1}(\mathbb{G})$, then $\tilde{\Gamma}_{\mu}$ defines a normal *isomorphism of $\tilde{\mathcal{H}}_{\mu}$ onto $\mathcal{H}_{\mu} \rtimes_{\Gamma_{\mu}} \mathbb{G}=\left(\Gamma_{\mu}\left(\mathcal{H}_{\mu}\right) \cup\left(1 \otimes L^{\infty}\left(\hat{\mathbb{G}}^{\prime}\right)\right)\right)^{\prime \prime}$.

An important consequence, whose application to quantum information theory will be explored in the next chapter, is the following. 
Corollary 4.4.5. If $\mu \in \mathcal{P}_{1}(\mathbb{G})$, then $\tilde{\mathcal{H}}_{\mu}$ is a subalgebra of $\mathcal{B}\left(L^{2}(\mathbb{G})\right)$ if and only if $\mathcal{H}_{\mu}$ is a subalgebra of $L^{\infty}(\mathbb{G})$.

Proof. If $\mathcal{H}_{\mu}$ is a subalgebra of $L^{\infty}(\mathbb{G})$, then $\mathcal{H}_{\mu} \rtimes_{\Gamma_{\mu}} \mathbb{G}$ is a subalgebra of $L^{\infty}(\mathbb{G}) \bar{\otimes} \mathcal{B}\left(L^{2}(\mathbb{G})\right)$. Hence, $\tilde{\Gamma}_{\mu}: \tilde{\mathcal{H}}_{\mu} \rightarrow L^{\infty}(\mathbb{G}) \bar{\otimes} \mathcal{B}\left(L^{2}(\mathbb{G})\right)$ is an injective homomorphism with respect to both the Choi-Effros product, and the usual product in $\mathcal{B}\left(L^{2}(\mathbb{G})\right)$, thereby making $\tilde{\mathcal{H}}_{\mu}$ a subalgebra of $\mathcal{B}\left(L^{2}(\mathbb{G})\right)$. The reverse implication is clear as $\mathcal{H}_{\mu}$ is always a von Neumann subalgebra of $\tilde{\mathcal{H}}_{\mu}$.

In the commutative case, Theorem 4.4.4 generalizes the main result in [40, Proposition 6.3] beyond second countable locally compact groups. In particular, if $\mu$ is a probability measure on $G$ such that $\mathcal{H}_{\mu}$ is a subalgebra of $L^{\infty}(G)$, then $\tilde{\mathcal{H}}_{\mu}=\mathcal{H}_{\mu} \vee \mathcal{R}(G)$. Indeed, since $\Theta^{l}(\mu)$ is an $\mathcal{R}(G)$-bimodule map, and its restriction to $L^{\infty}(G)$ is $\Phi_{\mu}$, we have $\mathcal{H}_{\mu} \cup \mathcal{R}(G) \subseteq \tilde{\mathcal{H}}_{\mu}$, which by Corollary 4.4.5 implies $\mathcal{H}_{\mu} \vee \mathcal{R}(G) \subseteq \tilde{\mathcal{H}}_{\mu}$. Conversely, in view of the equality $\rho(s) M_{f}=M_{\rho(s) f} \rho(s)$ for every $f \in L^{\infty}(G), s \in G$, and the inclusion $W_{a} \in L^{\infty}(G) \bar{\otimes} \mathcal{L}(G)$, we have

$$
\begin{aligned}
(1 \otimes \rho(s)) \Gamma_{a}\left(M_{f}\right) & =(1 \otimes \rho(s)) W_{a}^{*}\left(1 \otimes M_{f}\right) W_{a}=W_{a}^{*}\left(1 \otimes \rho(s) M_{f}\right) W_{a} \\
& =W_{a}^{*}\left(1 \otimes M_{\rho(s) f} \rho(s)\right) W_{a}=W_{a}^{*}\left(1 \otimes M_{\rho(s) f}\right) W_{a}(1 \otimes \rho(s)) \\
& =\Gamma_{a}\left(M_{\rho(s) f}\right)(1 \otimes \rho(s))
\end{aligned}
$$

for all $f \in L^{\infty}(G)$ and $s \in G$. Applying Theorem 2.4.2, we see that the von Neumann algebra $\mathcal{H}_{\mu} \rtimes_{\Gamma_{a}} \mathbb{G}_{a}=\left(\Gamma_{a}\left(\mathcal{H}_{\mu}\right) \cup(1 \otimes \mathcal{R}(G))\right)^{\prime \prime}$ is the weak* closure of operators 
of the form $\sum_{\imath=1}^{n} \Gamma_{a}\left(M_{f_{\imath}}\right)\left(1 \otimes \rho\left(s_{\imath}\right)\right)=\tilde{\Gamma}_{a}\left(\sum_{\imath=1}^{n} M_{f_{\imath}} \rho\left(s_{\imath}\right)\right), f_{\imath} \in \mathcal{H}_{\mu}, s_{\imath} \in G$. As a von Neumann algebra isomorphism, $\tilde{\Gamma}_{a}: \tilde{\mathcal{H}}_{\mu} \rightarrow \mathcal{H}_{\mu} \rtimes_{\Gamma_{a}} \mathbb{G}_{a}$ is a weak*-weak* homeomorphism. Thus,

$$
\tilde{\Gamma}_{a}\left(\tilde{\mathcal{H}}_{\mu}\right)=\mathcal{H}_{\mu} \rtimes_{\Gamma_{a}} \mathbb{G}_{a} \subseteq \tilde{\Gamma}_{a}\left(\mathcal{H}_{\mu} \vee \mathcal{R}(G)\right)
$$

which yields the reverse inclusion.

On the dual side, Theorem 4.4.4 generalizes the main result in [59, Theorem 4.8] beyond locally compact groups with the approximation property (see [29]). Since $\mathcal{H}_{\varphi}$ is always a von Neumann subalgebra of $\mathcal{L}(G)$ for every $\varphi \in \mathcal{P}_{1}(G)$, a similar argument as above gives $\tilde{\mathcal{H}}_{\varphi}=\mathcal{H}_{\varphi} \vee L^{\infty}(G)$.

In the language of quantum information, we have obtained a precise description of the fixed points of two intriguing classes of quantum channels. In fact, both classes yield new counter-examples to recently solved conjectures, as we shall see in the final chapter. 


\section{Chapter 5}

\section{Quantum Group Channels}

Here we explore some of the applications of the above results to quantum information theory. We study the relation between quantum channels arising from dual quantum groups, and show that on the level of quantum channels, quantum group duality is expressed as a commutation relation. We also present new classes of counter-examples to recently solved conjectures concerning fixed point algebras in infinite dimensions, and the convex structure of the set of bistochastic channels. Finally, we explicitly compute the harmonic operators for the smallest nontrivial quantum group, the KacPaljutkin algebra [42].

\subsection{Dual Channels}

A distinguishing property of quantum channels coming from our representation is that they have "dual" counterparts acting on the same space over the quantum system. 
More precisely, if $\mathbb{G}$ is a locally compact quantum group with dual $\hat{\mathbb{G}}$, then the quantum channels lying in $\Theta^{l}\left(\mathcal{P}_{1}(\mathbb{G})\right)$ are dual to those lying in $\hat{\Theta}^{l}\left(\mathcal{P}_{1}(\hat{\mathbb{G}})\right)$. One manifestation of this duality is the following result, where for a set $\mathcal{S} \subseteq \mathcal{C B}\left(\mathcal{B}\left(L^{2}(\mathbb{G})\right)\right)$, we denote by $\mathcal{S}^{c}$ its commutant in $\mathcal{C B}\left(\mathcal{B}\left(L^{2}(\mathbb{G})\right)\right)$.

Theorem 5.1.1. [41, Theorem 5.1] Let $\mathbb{G}$ be a locally compact quantum group with dual $\hat{\mathbb{G}}$. Then we have

$$
\hat{\Theta}^{l}\left(C_{c b}^{l}\left(L^{1}(\hat{\mathbb{G}})\right)\right)=\Theta^{l}\left(C_{c b}^{l}\left(L^{1}(\mathbb{G})\right)\right)^{c} \cap \mathcal{C}{\mathcal{B}_{L^{\infty}}^{\sigma}(\mathbb{G})^{\prime}}^{\prime}\left(\mathcal{B}\left(L^{2}(\mathbb{G})\right)\right)
$$

Corollary 5.1.2. Let $\mathbb{G}$ be a co-amenable locally compact quantum group with dual $\hat{\mathbb{G}}$. Then we have

$$
\hat{\Theta}^{l}(\mathcal{P}(\hat{\mathbb{G}}))=\Theta^{l}(\mathcal{P}(\mathbb{G}))^{c} \cap \mathcal{C} \mathcal{P}_{L^{\infty}(\mathbb{G})^{\prime}}^{\sigma}\left(\mathcal{B}\left(L^{2}(\mathbb{G})\right)\right)
$$

Proof. The inclusion " $\subseteq$ " follows directly from Theorem 5.1.1 and the definition of $C_{c b}^{l}\left(L^{1}(\hat{\mathbb{G}})\right)^{+}($cf. $\S 4.3 .2)$. Conversely, using [36, Theorem 4.2] and [5, Theorem 3.1], co-amenability implies

$$
C_{c b}^{l}\left(L^{1}(\mathbb{G})\right) \cong M(\mathbb{G}) \cong C_{u}(\mathbb{G})^{*}=\operatorname{span}(\mathcal{P}(\mathbb{G}))
$$

Hence, $\Theta^{l}\left(C_{c b}^{l}\left(L^{1}(\mathbb{G})\right)\right)=\operatorname{span} \Theta^{l}(\mathcal{P}(\mathbb{G}))$, so if $\Phi \in \mathcal{C P}_{L^{\infty}(\mathbb{G})^{\prime}}^{\sigma}\left(\mathcal{B}\left(L^{2}(\mathbb{G})\right)\right)$ commutes with $\Theta^{l}(\mathcal{P}(\mathbb{G}))$, then it necessarily commutes with $\Theta^{l}\left(C_{c b}^{l}\left(L^{1}(\mathbb{G})\right)\right)$, making $\Phi$ an 
element of $\hat{\Theta}^{l}(\mathcal{P}(\hat{\mathbb{G}}))$ by Theorem 5.1.1.

Definition 5.1.3. Let $H$ be a Hilbert space, and let $M$ be a von Neumann subalgebra of $\mathcal{B}(H)$. We say that a quantum channel $\Phi: \mathcal{B}(H) \rightarrow \mathcal{B}(H)$ is $M$-noiseless if it is an $M$-bimodule map. Note that $\Phi$ is $M$-noiseless if and only if its Kraus operators belong to $M^{\prime}[28]$.

From the viewpoint of quantum information, Corollary 5.1.2 says that dual quantum groups give rise to commuting quantum channels. Moreover, any $\mathbb{G}^{\prime}$-noiseless channel commuting with quantum group channels arising from $\mathbb{G}$, is necessarily a quantum group channel arising from $\hat{\mathbb{G}}$.

\subsection{Commutative and Co-Commutative Quantum}

\section{Groups}

Definition 5.2.1. Let $G$ be a locally compact group. A function $f \in L^{\infty}(G)$ is said to be left uniformly continuous if for every $\varepsilon>0$, there exists a neighborhood $U$ of the identity such that $\left\|l_{s} f-f\right\|_{\infty}<\varepsilon$ for all $s \in U$, where $l_{s} f(t)=f(s t), t \in G$. The set of such functions is denoted $L U C(G)$. We say that a family of functions $\left(f_{\alpha}\right)_{\alpha \in A}$ in $L^{\infty}(G)$ is equi-left uniformly continuous if for every $\varepsilon>0$, there exists a neighborhood $U$ of the identity such that $\left\|l_{s} f_{\alpha}-f_{\alpha}\right\|_{\infty}<\varepsilon$ for all $s \in U, \alpha \in A$. 
For a locally compact group $G$, recall that the representation (4.1) of the measure algebra is given by

$$
\left\langle\Theta^{l}(\mu)(x), \rho\right\rangle=\int_{G}\left\langle\lambda(s)^{*} x \lambda(s), \rho\right\rangle d \mu(s),
$$

for $\mu \in M(G), x \in \mathcal{B}\left(L^{2}(G)\right)$, and $\rho \in \mathcal{T}\left(L^{2}(G)\right)$. Let $M_{1}^{+}(G)$ denote the set probability measures in $M(G)$.

Proposition 5.2.2. If $\mu \in M_{1}^{+}(G)$, then $\Theta^{l}(\mu)$ is a bistochastic quantum channel on $\mathcal{B}\left(L^{2}(G)\right)$

Proof. Clearly $\Theta^{l}(\mu)$ is completely positive and unital, so it remains to show that it is trace preserving. Let $\left(e_{2}\right)_{\imath \in I}$ be an orthonormal basis for $L^{2}(G)$, and let $\rho \in \mathcal{T}\left(L^{2}(G)\right)$. Then $w^{*}-\sum_{\imath \in I} \theta_{e_{\imath}, e_{\imath}}=1$, and

$$
\begin{aligned}
\operatorname{tr}\left(\Theta^{l}(\mu)(\rho)\right)-\operatorname{tr}(\rho) & =\sum_{\imath \in I}\left\langle\Theta^{l}(\mu)(\rho)-\rho, \theta_{e_{2}, e_{2}}\right\rangle \\
& =\sum_{\imath \in I} \int_{G}\left(\left\langle\lambda(s)^{*} \rho \lambda(s), \theta_{e_{2}, e_{\imath}}\right\rangle-\operatorname{tr}(\rho)\right) d \mu(s) \\
& =\sum_{\imath \in I} \int_{G}\left(\left\langle\rho, \lambda(s) \theta_{e_{\imath}, e_{2}} \lambda(s)^{*}\right\rangle-\operatorname{tr}(\rho)\right) d \mu(s) .
\end{aligned}
$$

Let $\mathcal{F}(I)$ denote the directed set of finite subsets of $I$. Defining

$$
f_{A}(s)=\sum_{\imath \in A}\left(\left\langle\rho, \lambda(s) \theta_{e_{\imath}, e_{2}} \lambda(s)^{*}\right\rangle-\operatorname{tr}(\rho)\right), \quad A \in \mathcal{F}(I), s \in G
$$


we see that $\left(f_{A}\right)_{A \in \mathcal{F}(I)}$ is an equi-LUC family satisfying $\sup _{A}\left\|f_{A}\right\|_{\infty}<\infty$ and $f_{A} \rightarrow 0$ pointwise. Hence, $f_{A} \rightarrow 0$ uniformly on compact sets [57]. Now, using the regularity of $\mu$, one can interchange the sum and the integral to obtain

$$
\begin{aligned}
\operatorname{tr}\left(\Theta^{l}(\mu)(\rho)\right)-\operatorname{tr}(\rho) & =\int_{G} \sum_{\imath \in I}\left(\left\langle\rho, \lambda(s) \theta_{e_{2}, e_{2}} \lambda(s)^{*}\right\rangle-\operatorname{tr}(\rho)\right) d \mu(s) \\
& =\int_{G} \sum_{\imath \in I}\left(\left\langle\lambda(s)^{*} \rho \lambda(s), \theta_{e_{2}, e_{\imath}}\right\rangle-\operatorname{tr}(\rho)\right) d \mu(s) \\
& =\int_{G}\left(\left\langle\lambda(s)^{*} \rho \lambda(s), 1\right\rangle-\operatorname{tr}(\rho)\right) d \mu(s) \\
& =0 .
\end{aligned}
$$

Since $\rho \in \mathcal{T}\left(L^{2}(G)\right)$ was arbitrary, the result follows.

From classical harmonic function theory, it is known that every probability measure $\mu$ on a compact or locally compact abelian group is Choquet-Deny, meaning that any $\mu$-harmonic function $f \in L^{\infty}(G)$ is necessarily constant on the (left) cosets of $G_{\mu}$, the closed subgroup generated by the support of $\mu$. In this case, $\tilde{\mathcal{H}}_{\mu}=L^{\infty}\left(G / G_{\mu}\right) \vee \mathcal{R}(G)$ is a subalgebra of $\mathcal{B}\left(L^{2}(G)\right)$ (§4.4). We say that $\mu$ is adapted if $G_{\mu}=G$.

On dual side, given $\varphi \in \mathcal{P}_{1}(G)$, there exists a cyclic unitary representation $(\pi, \xi)$ such that $\varphi(s)=\langle\pi(s) \xi, \xi\rangle, s \in G$. Letting $\left(e_{\imath}\right)_{\imath \in I}$ be an orthonormal basis for $H_{\pi}$, 
and $\xi_{\imath}(s)=\left\langle e_{\imath}, \pi(s) \xi\right\rangle$, for $i \in I, s \in G$, it follows that

$$
\hat{\Theta}(\varphi)(x)=w^{*}-\sum_{\imath \in I} M_{\xi_{2}} x M_{\xi_{2}}^{*}, \quad x \in \mathcal{B}\left(L^{2}(G)\right)
$$

By Proposition 2.5.3, $\hat{\Theta}(\varphi)$ also defines a bistochastic quantum channel on $\mathcal{B}\left(L^{2}(G)\right.$ ). It is known that the set $G_{\varphi}=\{s \in G: \varphi(s)=1\}$ is a closed subgroup of $G$, and that $\mathcal{H}_{\varphi}=\mathcal{R}\left(G_{\varphi}\right)[14, \S 3.2]$. Hence, $\tilde{\mathcal{H}}_{\varphi}=\mathcal{R}\left(G_{\varphi}\right) \vee L^{\infty}(G)$ (§4.4). Following [59, Definition 2.3] we say that $\varphi$ is adapted if $G_{\varphi}=\{e\}$. Note that here we use the right version of [14].

When $G$ is locally compact abelian with Pontrjagin dual $\hat{G}$, the Fourier-Stieltjes transform provides a multiplicative linear injection from $M(G)^{+}$onto $\mathcal{P}(G)[25, \S 4.2]$. Denoting the image of $\mu \in M(G)_{1}^{+}$by $\hat{\mu}$, we may view the channel $\hat{\Theta}(\hat{\mu})$ as the "Fourier transform" of $\Theta^{l}(\mu)$.

For example, consider $G=\mathbb{Z}_{2}$. Any $\mu \in M_{1}^{+}\left(\mathbb{Z}_{2}\right)$ can be written as a convex combination of Dirac masses: $\mu=\mu(0) \delta_{0}+\mu(1) \delta_{1}$, in which case identifying $\hat{\mathbb{Z}}_{2}$ with $\mathbb{Z}_{2}$ yields $\hat{\mu}=\mu(0) \chi^{(0)}+\mu(1) \chi^{(1)}$, where $\chi^{(0)}$ and $\chi^{(1)}$ are the trivial, and sign character, respectively. Then

$$
\Theta^{l}(\mu)(\rho)=\mu(0) \rho+\mu(1) X \rho X^{*}
$$

and

$$
\hat{\Theta}(\hat{\mu})(\rho)=\mu(0) \rho+\mu(1) Z \rho Z^{*},
$$


where $X=\left(\begin{array}{ll}0 & 1 \\ 1 & 0\end{array}\right), Z=\left(\begin{array}{rr}1 & 0 \\ 0 & -1\end{array}\right)$, and $\rho \in \mathcal{B}\left(\mathbb{C}^{2}\right)$. We encountered the first channel in $§ 2.5$. It is known as the bit flip channel, while the second is called the phase flp channel as it induces a (local) phase flip on qubits. Both channels are of major importance in the theory of quantum error correction [52], which focuses on detecting and eliminating errors induced by the environment during a quantum computation.

Although the fixed points of these channels may be determined by rather elementary methods, computıng the fixed points of general quantum (or "noisy") channels is a central problem in quantum error correction (see [35] and the references therein). There are known algorithms for computing the fixed points of bistochastic channels in finite dimensions [85], but for certain cases, a more direct and efficient method not only avoids lengthy computation, but provides extra insight into the internal structure of the noise. With the aid of Theorem 4.4.4, we can obtain explicit descriptions of the fixed points of our classes of channels.

Example 5.2.3. Let $n \in \mathbb{N}$. Taking $G$ to be the Cartesian product of $n$ copies of $\mathbb{Z}_{2}$, denoted $\left(\mathbb{Z}_{2}\right)^{n}$, yields the $n$-qubit bit flip and phase flip channels. Indeed, as $\lambda\left(s_{1}, \ldots, s_{n}\right)=\otimes_{\imath=1}^{n} \lambda\left(s_{\imath}\right)$ for all $s=\left(s_{1}, \ldots, s_{n}\right) \in\left(\mathbb{Z}_{2}\right)^{n}$ and $M_{f \otimes g}=M_{f} \otimes M_{g}$ for all $f, g \in L^{\infty}\left(\left(\mathbb{Z}_{2}\right)^{n}\right)$, we may write

$$
\Theta^{l}(\mu)(\rho)=\sum_{s \in\left(\mathbb{Z}_{2}\right)^{n}} \mu(s) X_{s} \rho X_{s}^{*}
$$


and

$$
\hat{\Theta}(\hat{\mu})(\rho)=\sum_{s \in\left(\mathbb{Z}_{2}\right)^{n}} \mu(s) Z_{s} \rho Z_{s}^{*}
$$

where $X_{s}=\otimes_{\imath=1}^{n} \lambda\left(s_{\imath}\right)$ and $Z_{s}=\otimes_{\imath=1}^{n} M_{\chi^{s_{\imath}}}$, with $\chi^{s_{2}}$ the character on $\mathbb{Z}_{2}$ corresponding to $s_{\imath}$, and $s=\left(s_{1}, \ldots, s_{n}\right) \in\left(\mathbb{Z}_{2}\right)^{n}$. For instance, $\Theta^{l}(\mu)$ flips the first two qubits with probability $\mu(1,1,0, \ldots, 0)$.

The fixed points of these dual channels will clearly depend on the support of $\mu$. If $\mu$ is adapted, then $\tilde{\mathcal{H}}_{\mu}=\mathcal{R}\left(\left(\mathbb{Z}_{2}\right)^{n}\right)=\mathcal{L}\left(\left(\mathbb{Z}_{2}\right)^{n}\right)$, and $\tilde{\mathcal{H}}_{\hat{\mu}}=L^{\infty}\left(\left(\mathbb{Z}_{2}\right)^{n}\right)$. In this case, the channels share no common fixed points besides the trivial subspace $\mathbb{C} 1$. If $G_{\mu} \neq G$, then there are nonconstant $\mu$-harmonic functions $f \in L^{\infty}\left(\left(\mathbb{Z}_{2}\right)^{n}\right)$ for which $M_{f} \in \tilde{\mathcal{H}}_{\mu} \cap \tilde{\mathcal{H}}_{\hat{\mu}}$, namely those which are constant on the cosets of $G_{\mu}$. As noted in [59, Proposition 2.1] $G_{\mu} \neq G$ is equivalent to $G_{\hat{\mu}} \neq\{e\}$, so there are nontrivial translations $\rho(s) \in \tilde{\mathcal{H}}_{\mu} \cap \tilde{\mathcal{H}}_{\hat{\mu}}$. We therefore see that for nonadapted measures, we can "sneak" in extra information that will not be effected by either bit flips nor phase flips. In fact, the entire von Neumann algebra $L^{\infty}\left(G / G_{\mu}\right) \vee \mathcal{R}\left(G_{\hat{\mu}}\right)$ is left invariant under both channels.

A similar analysis can be done using the group $\left(\mathbb{Z}_{d}\right)^{n}, n, d \in \mathbb{N}$, giving the fixed points for the bit flip and phase flip channels on qudits, i.e., states on $\mathbb{C}^{d}$. 


\subsubsection{Fixed Point Algebras}

If $H$ is a finite dimensional Hilbert space, it is known that any bistochastic quantum channel $\Phi: \mathcal{B}(H) \rightarrow \mathcal{B}(H)$ satisfies

$$
\operatorname{Fix}(\Phi)=\left\{a_{\imath}, a_{\imath}^{*}\right\}^{\prime}
$$

where $\left(a_{\imath}\right)$ are the Kraus operators of $\Phi$. In particular, the fixed point set is always a subalgebra of $\mathcal{B}(H)[47$, Theorem 2.1].

It was shown in $[3, \S 4]$ that the infinite dimensional generalization is false. However, to our knowledge, the only known counter-examples are of the form $\Theta^{l}(\mu)$ for some $\mu \in M(G)$, where $G$ is an infinite discrete group (see also [55, §4]). By Corollary 4.4.5, we know that for general locally compact groups $G$, the fixed points of $\Theta^{l}(\mu)$ form a subalgebra of $\mathcal{B}\left(L^{2}(G)\right)$ if and only if $\mu$ is a Choquet-Deny measure, which in the adapted case means it has trivial harmonic functions. Therefore, using the well-known result in [45, Theorem 4.2], which states that any adapted measure on a nonamenable group has nontrivial harmonic functions, we immediately obtain the following.

Proposition 5.2.4. Let $\mu$ be an adapted probability measure on a nonamenable group G. Then $\tilde{\mathcal{H}}_{\mu}$ is not a subalgebra of $\mathcal{B}\left(L^{2}(G)\right)$. 
Analogous to the finite-dimensional case, it was shown in [55, Theorem 3.1] that any bistochastic channel $\Phi: \mathcal{B}(H) \rightarrow \mathcal{B}(H)$ on a separable Hilbert space $H$, satisfies

$$
\operatorname{Fix}(\Phi) \cap \mathcal{K}(H)=\left\{a_{i}, a_{i}^{*}\right\}^{\prime} \cap \mathcal{K}(H)
$$

where $\left(a_{i}\right)$ are the Kraus operators of $\Phi$, and $\mathcal{K}(H)$ is the ideal of compact operators. The following characterizes the above intersection for adapted probability measures.

Proposition 5.2.5. Let $\mu$ be an adapted probability measure on a locally compact group $G$. If $G$ is compact, then $\tilde{\mathcal{H}}_{\mu} \cap \mathcal{K}\left(L^{2}(G)\right)=C_{\rho}^{*}(G)$. If $G$ is not compact, then $\tilde{\mathcal{H}}_{\mu} \cap \mathcal{K}\left(L^{2}(G)\right)=\{0\}$.

Proof. If $G$ is compact, then $\mu$ is Choquet-Deny, so $\tilde{\mathcal{H}}_{\mu}=\mathcal{R}(G)(\S 4.4)$. But then, by $\left[13\right.$, Proposition 2.2], we have $\tilde{\mathcal{H}}_{\mu} \cap \mathcal{K}\left(L^{2}(G)\right)=\mathcal{R}(G) \cap \mathcal{K}\left(L^{2}(G)\right)=C_{\rho}^{*}(G)$, the (right) reduced $C^{*}$-algebra of $G$ (see equation (2.2)).

Suppose $G$ is not compact. If $x \in \tilde{\mathcal{H}}_{\mu} \cap \mathcal{K}\left(L^{2}(G)\right)$, then $x \in \tilde{\mathcal{H}}_{\mu^{n}} \cap \mathcal{K}\left(L^{2}(G)\right)$ for all $n \in \mathbb{N}$ as $\Theta^{l}$ is a homomorphism. Hence, for all $n \in \mathbb{N}$ and $\rho \in \mathcal{T}\left(L^{2}(G)\right)$, we have

$$
\langle x, \rho\rangle=\left\langle\Theta^{l}\left(\mu^{n}\right)(x), \rho\right\rangle=\int_{G}\left\langle\lambda(s)^{*} x \lambda(s), \rho\right\rangle d \mu^{n}(s) .
$$

Since $G$ is not compact, the convolution powers $\mu^{n}$ of $\mu$ converge to zero in the weak* topology of $M(G)$ [56]. Also, as $x$ is compact, the function $s \mapsto\left\langle\lambda(s)^{*} x \lambda(s), \rho\right\rangle$ lies in $C_{0}(G)$ for any $\rho \in \mathcal{T}\left(L^{2}(G)\right)$, as is easily seen by approximating $x$ with finite rank operators. Thus, by weak* convergence of $\mu^{n}$ to 0 , it follows from equation (5.2) that 
$\langle x, \rho\rangle=0$ for all $\rho \in \mathcal{T}\left(L^{2}(G)\right)$, which implies $x=0$.

Remark 5.2.6. In [55] Lim uses a slightly different notion of bistochasticity, namely where the operator sums $S_{1}=\sum_{\imath \in I} a_{\imath} a_{\imath}^{*}$ and $S_{2}=\sum_{\imath \in I} a_{\imath}^{*} a_{\imath}$ both converge to the identity in the strong operator topology, as opposed to the weak* topology. Since the weak operator topology is equivalent to the weak* topology on bounded subsets of $\mathcal{B}(H)$ [62, Theorem 1.2.4], the strong operator convergence of $S_{1}$ and $S_{2}$ implies weak* convergence. Hence, by Proposition 2.5.3, our notion of bistochasticity is more general than the one used in [55].

Returning to finite dimensions, the success of equation (5.1), in part, motivated a new form of passive error correction known as noiseless subsystems [35]. This method relies on the well-known fact from operator algebras that any finite dimensional $C^{*}$ algebra is unitarily equivalent to a direct sum of amplified matrix algebras of the form

$$
\bigoplus_{k} I_{m_{k}} \otimes M_{n_{k}}(\mathbb{C}), \quad k, n_{k}, m_{k} \in \mathbb{N}
$$

Since the fixed points of a bistochastic channel form a von Neumann subalgebra of $\mathcal{B}(H)$, it has such a decomposition, so the idea is to determine its explicit structure, which usually models the noise present during a quantum computation. If the decomposition contains nontrivial tensor factors, i.e., if $n_{k}>1$ for some $k$, then information encoded into any state belonging to $M_{n_{k}}(\mathbb{C})$ will remain immune to the noise, eliminating the need for error correction. 
Recall that for any probability measure $\mu$ on a locally compact group $G$, the fixed points of $\Theta^{l}(\mu)$ always contain $\mathcal{R}(G)$. In particular, if $G$ is compact, we know that

$$
\mathcal{R}(G) \cong \bigoplus_{[\pi]} I_{d_{\pi}} \otimes M_{d_{\pi}}(\mathbb{C})
$$

where the sum is taken over all (equivalence classes of) irreducible representations of $G$, and $d_{\pi}$ is the dimension of the representation [25, Theorem 5.12]. For example, if $G=D_{4}$, the dihedral group of order 8 , we have

$$
\mathcal{R}\left(D_{4}\right) \cong \mathbb{C} \oplus \mathbb{C} \oplus \mathbb{C} \oplus \mathbb{C} \oplus\left(I_{2} \otimes M_{2}(\mathbb{C})\right)
$$

Thus, any state supported on the last tensor factor $M_{2}(\mathbb{C})$, will be immune to the effect of $\Theta^{l}(\mu)$ for every probability measure $\mu \in M\left(D_{4}\right)$.

In order to implement this procedure in the laboratory, one requires the precise spatial decomposition of the Hilbert space giving rise to the decomposition (5.3) [35]. Fortunately, for our channels, this is simply given by the coefficient functions of the irreducible representations of $G$ by the Peter-Weyl theorem [25, Theorem 5.12]. Hence, we arrive at a new class of quantum channels (i.e., error models) whose noiseless subsystems may be computed explicitly using well known representation theory. 


\subsubsection{The Asymptotic Quantum Birkoff Conjecture}

Definition 5.2.7. A matrix $A \in M_{d}(\mathbb{R})$ is stochastic if $A_{\imath \jmath} \geq 0$ for all $i, j$ and $\sum_{\imath=1}^{d} A_{\imath \jmath}=1$ for every $j$. If, in addition, $\sum_{\jmath=1}^{d} A_{\imath \jmath}=1$ for every $i$, then $A$ is said to be bistochastic.

Letting $\Delta_{d}$ denote the probability simplex in $d$-dimensions, that is, the set of vectors $x=\left(x_{1}, \ldots, x_{d}\right) \in \mathbb{R}^{d}$ such that $x_{\imath} \geq 0$ for all $i$ and $\|x\|_{1}=1$, we see that stochastic matrices $A \in M_{d}(\mathbb{R})$ induce linear maps $A: \Delta_{d} \rightarrow \Delta_{d}$, where $A$ is bistochastic if and only if it fixes the uniform probability distribution $\left(\frac{1}{d}, \ldots, \frac{1}{d}\right)$. Bistochastic matrices therefore play an important role in dynamical probability theory, and have been intensively studied $[31,67]$. Their geometry is described by the following major theorem.

Theorem 5.2.8 (Birkoff's Theorem). [10] The set of $d \times d$ bistochastic matrices is a convex set whose extreme points are the $d$ ! permutation matrices.

In the quantum setting, states play the role of probability distributions, making bistochastic channels the quantum analogue of bistochastic matrices. We denote the set of bistochastic channels in dimension $d$ by $\mathcal{B I S}_{d}$. Reasoning by pure analogy, one would expect the extreme points of this convex set to be the unitary conjugations. For $d=2$ this is the case, but in general it is false [53, Theorem 1]. Shortly after the 1993 result of [53], it was conjectured that as the number of particles of a quantum system grows, bistochastic channels behave more and more like random unitaries. Formally speaking, if $\mathcal{R} \mathcal{U}\left(M_{d}(\mathbb{C})\right)$ denotes the (closed) convex hull of unitary conjugations in dimension $d$, then the asymptotıc quantum Birkoff conjecture states that 
any bistochastic channel $\Phi: M_{d}(\mathbb{C}) \rightarrow M_{d}(\mathbb{C})$ satisfies

$$
\lim _{n \rightarrow \infty}\left\|\Phi^{\otimes^{n}}-\mathcal{R} \mathcal{U}\left(M_{d}(\mathbb{C})^{\otimes^{n}}\right)\right\|_{c b}=0 .
$$

This conjecture remained open until this year, when Haagerup and Musat provided a counter-example using nonfactorizable maps $[30, \S 3]$. In what follows, we will obtain an new class of counter-examples arising from the representation of $M_{c b} A(G)$. To this end, we must first construct a $\varphi \in \mathcal{P}_{1}(G)$ for which $\hat{\Theta}(\varphi)$ is not random unitary. If $G$ is a finite group, then any $\varphi \in \mathcal{P}_{1}(G)$ with cyclic representation $(\pi, \xi)$ determines a $|G| \times|G|$ matrix $C_{\varphi}$, whose $(s, t)$ entry is given by $\varphi\left(s^{-1} t\right)=\langle\pi(t) \xi, \pi(s) \xi\rangle$. Since $\varphi$ is positive definite of norm one, $C_{\varphi}$ is a positive semi-definite matrix with diagonal entries equal to one, that is, a correlation matrix [7]. In fact, the unital quantum channel $\hat{\Theta}(\varphi)$ is completely determined by $C_{\varphi}$ in the following sense:

$$
\hat{\Theta}(\varphi)(x)=C_{\varphi} \circ_{S} x, \quad x \in \mathcal{B}\left(L^{2}(G)\right)
$$

where $\circ_{S}$ denotes the Schur (or pointwise) product of matrices. Such channels are often referred to as Schur maps because of their connection with the Schur product.

Let $\mathbb{C}_{d}$ denote the convex set of $d \times d$ complex correlation matrices, and let $\mathcal{E}\left(\mathbb{C}_{d}\right)$ denote its extreme points. The following two characterizations of $\mathcal{E}\left(\mathbb{C}_{d}\right)$ will be important for us. 
Theorem 5.2.9. [54, Theorem 1] Let $A$ be a $d \times d$ correlation matrix of rank $r$, and let $A=X X^{*}$ be its canonical decomposition, where $X \in M_{d \times r}(\mathbb{C})$. Then $A \in \mathcal{E}\left(\mathbb{C}_{d}\right)$ if and only if

$$
\operatorname{span}\left\{\theta_{x_{j}, x_{j}}: 1 \leq j \leq d\right\}=\mathcal{H}_{r}
$$

where $x_{j}$ is the $j^{\text {th }}$ column of $X^{*}$, and $\mathcal{H}_{r}$ is the real linear space of $r \times r$ hermitian matrices.

Theorem 5.2.10. [7, Lemma 2.4] Let $A$ be a $d \times d$ correlation matrix, and let $S_{A}: M_{d}(\mathbb{C}) \rightarrow M_{d}(\mathbb{C})$ be its corresponding bistochastic Schur map. Then $A \in \mathcal{E}\left(\mathbb{C}_{d}\right)$ if and only if $S_{A} \in \mathcal{E}\left(\mathcal{B I S}_{d}\right)$.

In the case of finite groups $G$, the matrix $C_{\varphi}$ is simply the Gram matrix of the set of vectors $\{\pi(s) \xi: s \in G\}$, where $\varphi=(\pi, \xi) \in \mathcal{P}_{1}(G)$. Hence, in the decomposition $C_{\varphi}=X X^{*}$, we may take $X^{*}$ to be the matrix whose columns are precisely the vectors $\pi(s) \xi, s \in G$.

Proposition 5.2.11. Let $G$ be a finite group, and let $\varphi=(\pi, \xi) \in \mathcal{P}_{1}(G)$. If

$$
\mathcal{H}_{r_{\varphi}}=\operatorname{span}\left\{\theta_{\pi(s) \xi, \pi(s) \xi}: s \in G\right\}
$$

where $r_{\varphi}=\operatorname{rank}\left(C_{\varphi}\right)$, then the following are equivalent:

1. $\hat{\Theta}(\varphi)$ is random unitary;

2. $\varphi$ is a character. 
Proof. Since $\varphi$ satisfies equation (5.5), Theorem 5.2.9 implies that $C_{\varphi} \in \mathcal{E}\left(\mathbb{C}_{|G|}\right)$. Hence, by Theorem 5.2.10, $\hat{\Theta}(\varphi) \in \mathcal{E}\left(\mathcal{B I} \mathcal{S}_{|G|}\right)$. So, if $\hat{\Theta}(\varphi)$ is random unitary, it is given by conjugation with a single unitary $U \in \mathcal{B}\left(L^{2}(G)\right)$. As $\hat{\Theta}(\varphi)$ is an $L^{\infty}(G)$ bimodule map, there exists $\chi \in L^{\infty}(G)$ such that $U=M_{\chi}$ and $|\chi(s)|=1$ for all $s \in G$ [28]. But then, recalling the action of $\hat{\Theta}(\varphi)$ on $\mathcal{R}(G)(\S 4.1)$, and the formula $\rho(s) M_{f}=M_{\rho(s) f} \rho(s)$ for $f \in L^{\infty}(G)$ and $s \in G$, we have

$$
\hat{\Theta}(\varphi)(\rho(s))=\varphi(s) \rho(s)=M_{\chi} \rho(s) M_{\chi}^{*}=M_{\chi} M_{\rho(s) \bar{\chi}} \rho(s)
$$

for all $s \in G$. Thus, $\varphi(s)=\chi(t) \overline{\chi(t s)}$ for all $s, t \in G$ implying $|\varphi(s)|=1$ for every $s \in G$, which, by [34, Theorem 32.7] makes $\varphi$ a character.

On the other hand, if $\varphi$ is a character, then by construction $\hat{\Theta}(\varphi)(x)=M_{\varphi}^{*} x M_{\varphi}$, $x \in \mathcal{B}\left(L^{2}(G)\right)$ with $M_{\varphi}$ unitary.

Example 5.2.12. Let $G=S_{3}$, the smallest nonabelian group. Then $S_{3}$ has a two dimensional irreducible representation $\pi: S_{3} \rightarrow \mathcal{B}\left(\mathbb{C}^{2}\right)$, given by its action on the triangle:

$$
\pi(e)=\left(\begin{array}{cc}
1 & 0 \\
0 & 1
\end{array}\right), \quad \pi(123)=\left(\begin{array}{cc}
e^{i 2 \pi / 3} & 0 \\
0 & e^{-i 2 \pi / 3}
\end{array}\right)
$$




$$
\begin{gathered}
\pi(132)=\left(\begin{array}{cc}
e^{-\imath 2 \pi / 3} & 0 \\
0 & e^{\imath 2 \pi / 3}
\end{array}\right), \quad \pi(12)=\left(\begin{array}{ll}
0 & 1 \\
1 & 0
\end{array}\right), \\
\pi(23)=\left(\begin{array}{cc}
0 & e^{-\imath 2 \pi / 3} \\
e^{\imath 2 \pi / 3} & 0
\end{array}\right), \quad \pi(13)=\left(\begin{array}{cc}
0 & e^{\imath 2 \pi / 3} \\
e^{-\imath 2 \pi / 3} & 0
\end{array}\right) .
\end{gathered}
$$

Letting $\left\{e_{1}, e_{2}\right\}$ denote the standard basis of $\mathbb{C}^{2}$, if $\xi=\frac{1}{\sqrt{5}}\left(-e_{1}+2 \imath e_{2}\right)$, for instance, one may easily verify by computation that the positive definite function $\varphi(s)=\langle\pi(s) \xi, \xi\rangle$, $s \in G$, satisfies equation (5.5). Therefore, by Proposition 5.2.11, the unital quantum channel $\hat{\Theta}(\varphi)$ is not random unitary as $\varphi$ is not a character on $S_{3}$.

Being dual to the random unitary channels $\Theta^{l}(\mu)$ arising from probability measures in $M\left(S_{3}\right)$, one would expect that the channel from Example 5.2.12 is "far away" from the set of all random unitaries in six dimensions. This is indeed the case, and with the help of factorizable maps [30], it is not too difficult to prove.

Definition 5.2.13. [30, Definition 1.1] Let $(M, \tau)$ and $(N, \omega)$ be von Neumann algebras equipped with normal faithful tracial states $\tau$ and $\omega$, respectively. A linear map $\Phi: M \rightarrow N$ is called a $(\tau, \omega)-$ Markov map if

1. $\Phi$ is completely positive,

2. $\Phi$ is unital, 
3. $\omega \circ \Phi=\tau$.

In particular, when $(M, \tau)=(N, \omega)$, we say that $\Phi$ is an $\tau-$ Markov map.

If $\Phi: M \rightarrow N$ is a $(\tau, \omega)$-Markov map, it was shown in [1, Lemma 2.5] that there exists a unique completely positive, unital map $\tilde{\Phi}: N \rightarrow M$ such that

$$
\tau(\tilde{\Phi}(y) x)=\omega(y \Phi(x)), \quad x \in M, \quad y \in N
$$

Definition 5.2.14. [30, Definition 1.3] A $(\tau, \omega)$-Markov map $\Phi: M \rightarrow N$ is called factorizable if there exists a von Neumann algebra $P$ equipped with a normal faithful tracial state $\chi$, and injective $*$-homomorphisms $J_{0}: M \rightarrow P$ and $J_{1}: N \rightarrow P$ such that $J_{0}$ is $(\tau, \chi)$-Markov and $J_{1}$ is $(\omega, \chi)$-Markov, satisfying, moreover, $\Phi=J_{0}^{*} \circ J_{1}$.

Remark 5.2.15. The definitions of $(\tau, \omega)$-Markov maps and factorizable maps in [30] do not assume the associated states are tracial. However, for our purposes, this provides no restriction as we will always be working with the unique normalized trace $\tau_{d}$ on $M_{d}(\mathbb{C}), d \geq 1$ (see $[30$, Remark $\left.1.4(\mathrm{a})]\right)$.

In what follows, a von Neumann algebra $M$ is said to be fintte if $x \in M$ and $x^{*} x=1$ imply $x x^{*}=1[62, \S 6.3]$. Note that a linear map $\Phi: M_{d}(\mathbb{C}) \rightarrow M_{d}(\mathbb{C})$ is a $\tau_{d}$-Markov map if and only if it is bistochastic. 
Theorem 5.2.16. [30, Theorem 2.2] Let $\Phi: M_{d}(\mathbb{C}) \rightarrow M_{d}(\mathbb{C})$ be a bistochastic channel written in the form

$$
\Phi(x)=\sum_{\imath=1}^{n} a_{\imath}^{*} x a_{\imath}, \quad x \in M_{d}(\mathbb{C})
$$

with $a_{1}, \ldots, a_{n}$ linearly independent. Then the following conditions are equivalent:

1. $\Phi$ is factorizable.

2. There exists a finite von Neumann algebra $N$, equipped with a normal faithful tracial state $\tau_{N}$, and a unitary operator $U \in M_{d}(\mathbb{C}) \otimes N$ such that

$$
\Phi(x)=\left(\iota \otimes \tau_{N}\right)\left(U^{*}(x \otimes 1) U\right), \quad x \in M_{d}(\mathbb{C})
$$

3. There exists a finte von Neumann algebra $N$, equipped with a normal faithful tracial state $\tau_{N}$, and $v_{1}, \ldots, v_{n} \in N$ such that $U:=\sum_{\imath=1}^{n} a_{\imath} \otimes v_{\imath}$ is unitary in $M_{d}(\mathbb{C}) \otimes N$, and

$$
\tau_{N}\left(v_{\imath}^{*} v_{\jmath}\right)=\delta_{\imath \jmath}, \quad 1 \leq i, j \leq n
$$

With this characterization, it is easy to see that unitary conjugations are factorizable, and that the set of factorizable maps on $M_{d}(\mathbb{C})$ is convex. Hence, random unitary channels are factorizable. In fact, they are precisely those factorizable channels for which the von Neumann algebra $N$ in Theorem 5.2.16 is abelian [30, Proposition 2.4]. 
Recall from $\S 4.3 .1$ that an element $\varphi \in \mathcal{P}_{1}(G)$ is an extreme point if and only if the associated representation $\pi_{\varphi}$ is irreducible. By injectivity of $\hat{\Theta}$, we see that this condition is necessary, but insufficient for $\hat{\Theta}(\varphi)$ to be extreme within the set of bistochastic channels. Indeed, taking $\pi: S_{3} \rightarrow \mathcal{B}\left(\mathbb{C}^{2}\right)$ as in Example 5.2.12, and defining $\varphi=(\pi, \xi) \in \mathcal{P}_{1}\left(S_{3}\right)$ with $\xi=e_{1}$, one can easily show that equation (5.5) is not satisfied. Hence, by Theorems 5.2.9 and 5.2.10, $\hat{\Theta}(\varphi)$ is not extreme. This motivates the following definition.

Definition 5.2.17. Let $G$ be a finite group. An element $\varphi \in \mathcal{P}_{1}(G)$ is maximally extreme if $\hat{\Theta}(\varphi)$ is an extreme point of $\mathcal{B I S}_{|G|}$.

Example 5.2.18. The positive definite function $\varphi=(\pi, \xi) \in \mathcal{P}_{1}\left(S_{3}\right)$ from Example 5.2.12 satisfies equation (5.5), and is therefore maximally extreme.

Proposition 5.2.19. Let $G$ be a finite group. If $\varphi \in \mathcal{P}_{1}(G)$ is maximally extreme with a cyclic representation $(\pi, \xi)$ satisfying $d_{\pi} \geq 2$, then $\hat{\Theta}(\varphi)$ is not factorizable.

Proof. Let $\left(e_{\imath}\right)_{\imath=1}^{d_{\pi}}$ be an orthonormal basis for $H_{\pi}$. Then defining $\xi_{\imath}(s)=\left\langle e_{\imath}, \pi(s) \xi\right\rangle$, $1 \leq i \leq d_{\pi}, s \in G$, we have

$$
\hat{\Theta}(\varphi)(x)=\sum_{i=1}^{d_{\pi}} M_{\xi_{2}} x M_{\xi_{2}}^{*}, \quad x \in \mathcal{B}\left(L^{2}(G)\right) .
$$

The set $\left\{M_{\xi_{2}}^{*}: 1 \leq i \leq d_{\pi}\right\}$ is linearly independent, for if $\sum_{\imath=1}^{d_{\pi}} \alpha_{i} M_{\xi_{2}}^{*}=0$, then 
$\sum_{\imath=1}^{d_{\pi}} \alpha_{\imath} \overline{\xi_{\imath}(s)}=0$ for all $s \in G$, and so by the definition of $\xi_{\imath}$, we have

$$
\left\langle\xi, \sum_{\imath=1}^{d_{\pi}} \overline{\alpha_{\imath}} \pi(s) e_{\imath}\right\rangle=0, \quad \forall s \in G
$$

As the subspace $V:=\operatorname{span}\left\{\sum_{\imath=1}^{d_{\pi}} \overline{\alpha_{\imath}} \pi(s) e_{\imath}: s \in G\right\}$ is $G$-invariant, equation (5.6) yields that $\pi(t) \xi \perp V$ for every $t \in G$, from which the cyclicity of $\xi$ implies that $V=\{0\}$.

Assuming $\hat{\Theta}(\varphi)$ is factorizable, we may then apply Theorem 5.2.16 to obtain a finite von Neumann algebra $N$, with normal faithful tracial state $\tau_{N}$, and elements $v_{1}, \ldots, v_{d_{\pi}}$ such that $U:=\sum_{\imath=1}^{d_{\pi}} M_{\xi_{2}}^{*} \otimes v_{\imath}$ is a unitary operator in $\mathcal{B}\left(L^{2}(G)\right) \otimes N$, and $\tau_{N}\left(v_{\imath}^{*} v_{\jmath}\right)=\delta_{\imath \jmath}, \quad 1 \leq \imath, \jmath \leq d_{\pi}$. Taking a cue from [30, Corollary 2.3], it follows that

$$
\sum_{\imath, \jmath=1}^{d_{\pi}} M_{\xi_{\imath}} M_{\xi_{\jmath}}^{*} \otimes\left(v_{\imath}^{*} v_{\jmath}-\delta_{\imath \jmath} 1_{N}\right)=U^{*} U-\left(\sum_{\imath=1}^{d_{\pi}} M_{\xi_{\imath}} M_{\xi_{2}}^{*}\right) \otimes 1_{N}=0
$$

If $\omega$ is a hermitian functional on $N$, so that $\omega\left(x^{*}\right)=\overline{\omega(x)}$ for all $x \in N$, applying $\iota \otimes \omega$ to the above equation yields

$$
\sum_{\imath, \jmath=1}^{d_{\pi}} \omega\left(v_{\imath}^{*} v_{\jmath}-\delta_{\imath \jmath} 1_{N}\right) M_{\xi_{2}} M_{\xi_{\jmath}}^{*}=0
$$


upon which setting $\omega_{\imath \jmath}=\omega\left(v_{\imath}^{*} v_{\jmath}-\delta_{\imath \jmath} 1_{N}\right)$ implies

$$
\begin{aligned}
0 & =\sum_{\imath, \jmath=1}^{d_{\pi}} \omega_{\imath \jmath} \xi_{\imath}(s) \overline{\xi_{\jmath}(s)} \\
& =\sum_{\imath, \jmath=1}^{d_{\pi}} \omega_{\imath \jmath}\left\langle e_{\imath}, \pi(s) \xi\right\rangle\left\langle\pi(s) \xi, e_{\jmath}\right\rangle \\
& =\sum_{\imath, \jmath=1}^{d_{\pi}} \omega_{\imath \jmath} \operatorname{tr}\left(\theta_{\pi(s) \xi, \pi(s) \xi} \theta_{e_{\imath}, e_{\jmath}}\right) \\
& =\operatorname{tr}\left(\theta_{\pi(s) \xi, \pi(s) \xi} \Omega\right)
\end{aligned}
$$

for all $s \in G$, where $\Omega$ is the $d_{\pi} \times d_{\pi}$ matrix with $(\imath, \jmath)$ entry equal to $\omega_{\imath \jmath}$. Since $\omega$ is a hermitian functional on $N$, and $\left(v_{\imath}^{*} v_{\jmath}-\delta_{\imath \jmath} 1_{N}\right)^{*}=v_{\jmath}^{*} v_{\imath}-\delta_{\jmath \imath} 1_{N}$, we see that $\Omega$ is a hermitian matrix satisfying $\operatorname{tr}\left(\theta_{\pi(s) \xi, \pi(s) \xi} \Omega\right)=0$ for all $s \in G$, which implies $\Omega=0$ by maximality of $\varphi$ (equation (5.5)). As every linear functional on $N$ is a linear combination of hermitian functionals, it follows that $\omega\left(v_{\imath}^{*} v_{\jmath}-\delta_{\imath \jmath} 1_{N}\right)=0$ for every $\omega \in N^{*}$, yielding $v_{\imath}^{*} v_{\jmath}=\delta_{\imath \jmath} 1_{N}$ for all $i, \jmath$. Since $d_{\pi} \geq 2$, we have in particular that $v_{1}^{*} v_{1}=v_{2}^{*} v_{2}=1_{N}$, making both $v_{1}$ and $v_{2}$ unitary operators since $N$ is finite. Thus, we have unitary operators satisfying $v_{1}^{*} v_{2}=0$, a contradiction.

Remark 5.2.20. After the proof of Proposition 5.2.19 was established, it was found that [53, Corollary 1] together with [30, Corollary 2.3] yields the same result.

In $[30$, Theorem 6.1$]$, it was also shown that any nonfactorizable map does not satisfy the asymptotic quantum Birkoff conjecture. The following corollary is therefore immediate. 
Corollary 5.2.21. Let $G$ be a finıte group. If $\varphi \in \mathcal{P}_{1}(G)$ is maxımally extreme with a cyclic representation $(\pi, \xi)$ satısfyıng $d_{\pi} \geq 2$, then $\hat{\Theta}(\varphi)$ does not satısfy the asymptotıc quantum Bırkoff conjecture.

Recall that the positive definite function $\varphi=(\pi, \xi)$ from Example 5.2.12 is maximally extreme, thereby giving a concrete counter-example to the asymptotic quantum Birkoff conjecture. To see that $\pi$ yields many counter-examples as $\xi$ varies over the unit sphere in $\mathbb{C}^{2}$, we turn to a geometric characterization of maximal extremity. We outline the construction for two-dimensional representations, but the theory extends naturally to arbitrary dimensions, as explained in the upcoming paper [16]. We also note that Helm and Strunz have studied this geometric representation and obtained a version of Proposition 5.2.22 [33].

Given a qubit $\rho \in \mathcal{D}\left(\mathbb{C}^{2}\right)$, we may write it as

$$
\rho=\frac{1}{2}\left(I_{2}+\overrightarrow{r_{\rho}} \cdot \vec{\sigma}\right)
$$

where $\vec{\sigma}:=(X, Y, Z)$ is the vector of Pauli matrices given by

$$
\begin{gathered}
X=\left(\begin{array}{ll}
0 & 1 \\
1 & 0
\end{array}\right), \quad Y=\left(\begin{array}{cc}
0 & -\imath \\
\imath & 0
\end{array}\right), \quad Z=\left(\begin{array}{cc}
1 & 0 \\
0 & -1
\end{array}\right), \\
\overrightarrow{r_{\rho}}:=(\operatorname{tr}(\rho X), \operatorname{tr}(\rho Y), \operatorname{tr}(\rho Z)) \in \mathbb{R}^{3},\left\|\overrightarrow{r_{\rho}}\right\| \leq 1, \text { and } \overrightarrow{r_{\rho}} \cdot \vec{\sigma}:=\operatorname{tr}(\rho X) X+\operatorname{tr}(\rho Y) Y+
\end{gathered}
$$

$\operatorname{tr}(\rho Z) Z$. This gives a one-to-one correspondence between quantum states in $\mathcal{D}\left(\mathbb{C}^{2}\right)$ and vectors in the closed unit ball of $\mathbb{R}^{3}$, known as the Bloch sphere representation 
$[60, \S 8.3 .2]$. One can easily show that $\rho$ is pure if and only if $\left\|\vec{r}_{\rho}\right\|=1$.

Let $G$ be a finite group, and $\pi: G \rightarrow \mathcal{B}\left(\mathbb{C}^{2}\right)$ be a unitary representation. Then $\pi$ induces an action of $G$ on the Bloch sphere via $(s, \rho) \mapsto \pi(s) \rho \pi(s)^{*}, s \in G, \rho \in$ $\mathcal{D}\left(\mathbb{C}^{2}\right)$. If $\xi$ is a unit vector in $\mathbb{C}^{2}$, we let $\overrightarrow{r_{s}}$ denote the vector in $\mathbb{R}^{3}$ corresponding to $\pi(s) \theta_{\xi, \xi} \pi(s)^{*}, s \in G$. Not surprisingly, the extremity of the positive definite function $\varphi=(\pi, \xi)$ is related to the geometry of the orbit of the pure state $\theta_{\xi, \xi}$.

Proposition 5.2.22. Let $G$ be a fintte group, and let $\varphi=(\pi, \xi) \in \mathcal{P}_{1}(G)$ with $d_{\pi}=2$. Then $\varphi$ is maximally extreme if and only if the affine span of $\left(\overrightarrow{r_{s}}\right)_{s \in G}$ is $\mathbb{R}^{3}$.

Proof. By Theorem 5.2.9, $\varphi$ is maximally extreme if and only if $\operatorname{span}\left\{\pi(s) \theta_{\xi, \xi} \pi(s)^{*} \mid\right.$ $s \in G\}=\mathcal{H}_{2}$, the real linear space of $2 \times 2$ hermitian matrices (see equation (5.5)). Endowed with the Hilbert-Schmidt inner product, defined by $\langle A, B\rangle_{H S}=\operatorname{tr}(A B)$, $A, B \in \mathcal{H}_{2}$, the space $\mathcal{H}_{2}$ becomes a Hilbert space upon which $\left\{I_{2}, X, Y, Z\right\}$ forms an orthogonal basis. For $s \in G$, and $A \in \mathcal{H}_{2}$, writing the Bloch sphere representation of $\theta_{\pi(s) \xi, \pi(s) \xi}$ yields

$$
\left\langle\theta_{\pi(s) \xi, \pi(s) \xi}, A\right\rangle_{H S}=\overrightarrow{R_{s}} \cdot \vec{a}
$$

with $\overrightarrow{R_{s}}:=\frac{1}{2}\left(1, \overrightarrow{r_{s}}\right)$ and $\vec{a}:=(\operatorname{tr}(A), \operatorname{tr}(A X), \operatorname{tr}(A Y), \operatorname{tr}(A Z))$ in $\mathbb{R}^{4}$, where $\cdot$ denotes the usual dot product. Hence, $\varphi$ is maximally extreme if and only if $\operatorname{span}\left\{\overrightarrow{R_{s}} \mid s \in\right.$ $G\}=\mathbb{R}^{4}$.

Now, suppose the affine span of $\left(\overrightarrow{r_{s}}\right)_{s \in G}$ is $\mathbb{R}^{3}$, and that there exists some nonzero $\vec{v}=\left(v_{0}, \overrightarrow{v_{1}}\right) \in \mathbb{R}^{4}$ orthogonal to $\overrightarrow{R_{s}}$ for all $s \in G$. Then $\vec{v} \cdot \overrightarrow{R_{s}}=\frac{1}{2}\left(v_{0}+\overrightarrow{v_{1}}\right.$. $\left.\overrightarrow{r_{s}}\right)=0$, which implies $\overrightarrow{v_{1}} \cdot \overrightarrow{r_{s}}=-v_{0}$ for all $s \in G$. But then, if $\left(\alpha_{s}\right)_{s \in G}$ are real 
numbers summing to 1 , we have $\overrightarrow{v_{1}} \cdot\left(\sum_{s \in G} \alpha_{s} \overrightarrow{r_{s}}\right)=-v_{0}$, and since the affine span of $\left(\overrightarrow{r_{s}}\right)_{s \in G}$ is $\mathbb{R}^{3}$, we get $\overrightarrow{v_{1}}=\left(-v_{0},-v_{0},-v_{0}\right)$, making $\vec{v}=v_{0}(1,-1,-1,-1)$. Writing $(-1,-1,-1)$ as the affine combination $\sum_{s \in G} \beta_{s} \overrightarrow{r_{s}}$, yields

$$
\vec{v}=v_{0}\left(\sum_{s \in G} \beta_{s}, \sum_{s \in G} \beta_{s} \overrightarrow{r_{s}}\right)=\sum_{s \in G} v_{0} \beta_{s}\left(1, \overrightarrow{r_{s}}\right)=\sum_{s \in G} 2 v_{0} \beta_{s} \overrightarrow{R_{s}}
$$

a contradiction.

Conversely, suppose $\varphi$ is maximally extreme. Then $\operatorname{span}\left\{\overrightarrow{R_{s}} \mid s \in G\right\}=\mathbb{R}^{4}$, and by injectivity of $\hat{\Theta}, \pi$ is irreducible. Thus, if $\left\{e_{1}, e_{2}\right\}$ is an orthonormal basis for $H_{\pi}$, Schur's orthogonality relations [25, Theorem 5.8] imply that

$$
\begin{aligned}
\frac{1}{|G|} \sum_{s \in G}\left\langle\theta_{\pi(s) \xi, \pi(s) \xi} e_{\jmath}, e_{\imath}\right\rangle & =\frac{1}{|G|} \sum_{s \in G}\left\langle e_{\jmath}, \pi(s) \xi\right\rangle\left\langle\pi(s) \xi, e_{\imath}\right\rangle \\
& =\frac{1}{|G|} \sum_{k, l=1}^{2} \sum_{s \in G}\left\langle e_{l}, \xi\right\rangle\left\langle\xi, e_{k}\right\rangle \overline{\left\langle\pi(s) e_{l}, e_{\jmath}\right\rangle}\left\langle\pi(s) e_{k}, e_{\imath}\right\rangle \\
& =\sum_{k, l=1}^{2}\left\langle e_{l}, \xi\right\rangle\left\langle\xi, e_{k}\right\rangle \frac{1}{2} \delta_{\imath \jmath} \delta_{k l} \\
& =\frac{1}{2} \delta_{\imath \jmath} \sum_{k=1}^{2}\left|\left\langle\xi, e_{k}\right\rangle\right|^{2} \\
& =\frac{1}{2} \delta_{\imath \jmath}
\end{aligned}
$$

for all $1 \leq i, \jmath \leq 2$. Hence, $\frac{1}{|G|} \sum_{s \in G} \theta_{\pi(s) \xi, \pi(s) \xi}=\frac{1}{2} I_{2}$, which implies $\frac{1}{|G|} \sum_{s \in G} \overrightarrow{r_{s}}=0$. By maximal extremity of $\varphi$, any vector $\vec{v} \in \mathbb{R}^{3}$ can be written as $\vec{v}=\sum_{s \in G} \alpha_{s} \overrightarrow{r_{s}}$, 
for some $\left(\alpha_{s}\right)_{s \in G}$ satisfying $\sum_{s \in G} \alpha_{s}=0$. But then

$$
\sum_{s \in G}\left(\frac{1}{|G|}+\alpha_{s}\right) \overrightarrow{r_{s}}=\sum_{s \in G} \frac{\overrightarrow{r_{s}}}{|G|}+\vec{v}=\vec{v}
$$

Thus, $\vec{v}$ belongs to the affine span of $\left(\overrightarrow{r_{s}}\right)_{s \in G}$.

For a visual example, consider the two-dimensional irreducible representation $\pi$ : $S_{3} \rightarrow \mathcal{B}\left(\mathbb{C}^{2}\right)$ from Example 5.2.12. As $\xi \in \mathbb{C}^{2}$ varies, we see that the orbit of $\theta_{\xi, \xi}$ varies accordingly in its geometric structure:

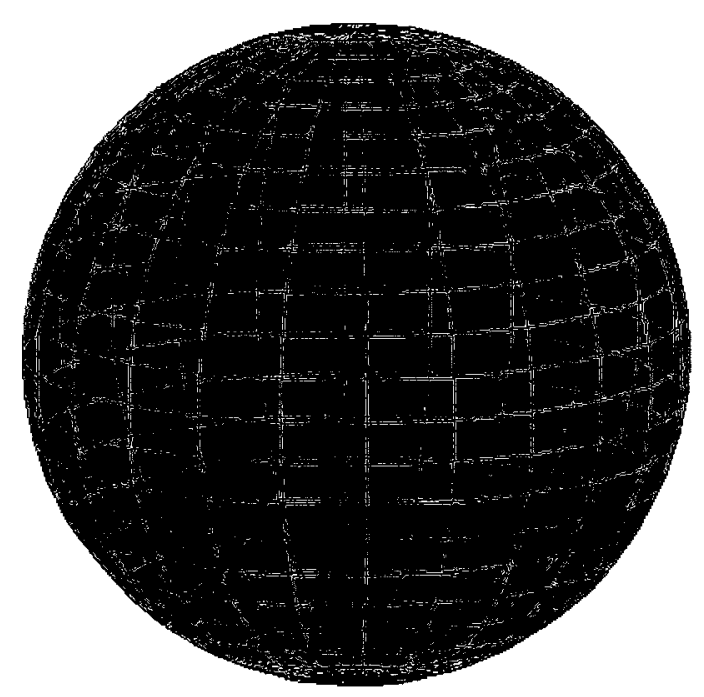

Figure 5.1: $\varphi=(\pi, \xi)$ with $\xi=\frac{1}{\sqrt{10}}(i, 3)$ is maximally extreme. Produced in Maple. 


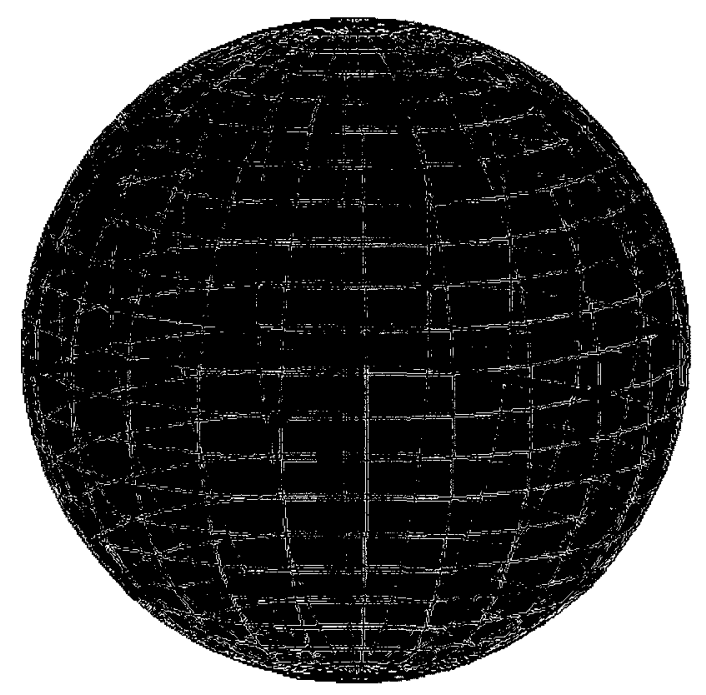

Figure 5.2: $\varphi=(\pi, \xi)$ with $\xi=\frac{1}{\sqrt{2}}(1, i)$ is not maximally extreme. Produced in Maple.

\subsection{The Kac-Paljutkin Algebra}

Historically, the first example of a locally compact quantum group which is neither commutative nor co-commutative was introduced by Kac and Paljutkin in 1965, and is known as the Kac-Paljutkin algebra [42]. One may realize this algebra through various constructions, but we shall use the method of "deformation by an abelian subgroup" developed by Landstad and Enock-Vainerman. See [22, §6.4] and the references therein for details.

Let $G_{8}$ denote the semi-direct product group $\left(\mathbb{Z}_{2} \times \mathbb{Z}_{2}\right) \rtimes_{\alpha} \mathbb{Z}_{2}$, where the action of $\mathbb{Z}_{2}$ is given by permutation of coordinates. We label the group elements in the natural binary order by

$$
G_{8}=\left\{e_{8}, z, a, a z, b, b z, a b, a b z\right\}
$$


where $e_{8}=(0,0,0), z=(0,0,1), a=(0,1,0), a z=(0,1,1), b=(1,0,0), b z=$ $(1,0,1), a b=(1,1,0)$, and $a b z=(1,1,1)$. Under the usual symmetric co-multiplication, $\mathcal{L}\left(G_{8}\right)$ becomes a co-commutative quantum group of dimension 8. To obtain the KacPaljutkin algebra, we deform the co-product $\Gamma_{s}$ using a particular unitary element $\Omega \in \mathcal{L}\left(G_{8}\right) \bar{\otimes} \mathcal{L}\left(G_{8}\right)$ in the following way.

Let $\omega \in L^{\infty}\left(\hat{\mathbb{Z}}_{2}{ }^{2} \times \hat{\mathbb{Z}}_{2}^{2}\right)$ be the function defined by $\omega(a, b)=\omega(b, a b)=\omega(a b, a)=$ $e^{i \pi / 4}, \omega\left(\chi^{(0,0)}, u\right)=\omega(u, u)=1$, and $\omega(u, t)=\overline{\omega(t, u)}$ where $u, t \in \hat{\mathbb{Z}}_{2}^{2}$ and $\chi^{(0,0)}$, $a=\chi^{(0,1)}$ and $b=\chi^{(1,0)}$ are the characters corresponding to $(0,0),(0,1)$ and $(1,0)$ in $\mathbb{Z}_{2}^{2}$, respectively. Next, let $\Omega$ be the unitary element in $\mathcal{L}\left(\mathbb{Z}_{2}^{2}\right) \bar{\otimes} \mathcal{L}\left(\mathbb{Z}_{2}^{2}\right)$, obtained via Fourier transform of $\omega$. Then, with the notation of equation $(2.1), \Omega=\left(\mathcal{F}^{-1}\right)^{*}(\omega)$, where $\mathcal{F}: L^{1}\left(\hat{\mathbb{Z}}_{2}^{2} \times \hat{\mathbb{Z}}_{2}^{2}\right) \rightarrow A\left(\mathbb{Z}_{2}^{2} \times \mathbb{Z}_{2}^{2}\right)$. So, for $\psi \in A\left(\mathbb{Z}_{2}^{2} \times \mathbb{Z}_{2}^{2}\right)$, we have

$$
\begin{aligned}
\langle\Omega, \psi\rangle & =\left\langle\omega, \mathcal{F}^{-1}(\psi)\right\rangle \\
& =\left\langle\omega, \frac{1}{16} \sum_{g, h \in \mathbb{Z}_{2}^{2}} \psi(g, h) \chi^{g} \chi^{h}\right\rangle \\
& =\frac{1}{16} \sum_{g, h \in \mathbb{Z}_{2}^{2}} \sum_{s, t \in \mathbb{Z}_{2}^{2}} \psi(g, h) \omega\left(\chi^{s}, \chi^{t}\right) \chi^{s}(g) \chi^{t}(h) \\
& =\frac{1}{16} \sum_{g, h \in \mathbb{Z}_{2}^{2}} \psi(g, h) \sum_{s, t \in \mathbb{Z}_{2}^{2}} \omega\left(\chi^{s}, \chi^{t}\right) \chi^{s}(g) \chi^{t}(h) \\
& =\left\langle\frac{1}{16} \sum_{g, h \in \mathbb{Z}_{2}^{2}}\left(\sum_{s, t \in \mathbb{Z}_{2}^{2}} \omega\left(\chi^{s}, \chi^{t}\right) \chi^{s}(g) \chi^{t}(h)\right) \lambda(g, h), \psi\right\rangle .
\end{aligned}
$$

Under the canonical embedding $\mathcal{L}\left(\mathbb{Z}_{2}^{2}\right) \bar{\otimes} \mathcal{L}\left(\mathbb{Z}_{2}^{2}\right) \hookrightarrow \mathcal{L}\left(G_{8}\right) \bar{\otimes} \mathcal{L}\left(G_{8}\right)$, we may therefore 
write

$$
\Omega=\sum_{g, h \in \mathbb{Z}_{2}^{2}} c(g, h) \lambda(g, e) \otimes \lambda(h, e),
$$

where $e$ denotes the identity in $\mathbb{Z}_{2}$, and $c(g, h)$ is the matrix of coefficients determined by

$$
c(g, h)=\frac{1}{16} \sum_{s, t \in \mathbb{Z}_{2}^{2}} \omega\left(\chi^{s}, \chi^{t}\right) \chi^{s}(g) \chi^{t}(h)
$$

Numerically, we obtain (see Appendix)

$$
[c(g, h)]=\left(\begin{array}{cccc}
\frac{5}{8}+\frac{3 \sqrt{2}}{16} & \frac{1}{8}-\frac{\sqrt{2}}{16} & \frac{1}{8}-\frac{\sqrt{2}}{16} & \frac{1}{8}-\frac{\sqrt{2}}{16} \\
\frac{1}{8}-\frac{\sqrt{2}}{16} & \frac{1}{8}-\frac{\sqrt{2}}{16} & -\frac{1}{8}+\frac{\sqrt{2}}{16}-i \frac{\sqrt{2}}{8} & i \frac{\sqrt{2}}{8}-\frac{1}{8}+\frac{\sqrt{2}}{16} \\
\frac{1}{8}-\frac{\sqrt{2}}{16} & i \frac{\sqrt{2}}{8}-\frac{1}{8}+\frac{\sqrt{2}}{16} & \frac{1}{8}-\frac{\sqrt{2}}{16} & -\frac{1}{8}+\frac{\sqrt{2}}{16}-i \frac{\sqrt{2}}{8} \\
\frac{1}{8}-\frac{\sqrt{2}}{16} & -\frac{1}{8}+\frac{\sqrt{2}}{16}-i \frac{\sqrt{2}}{8} & \frac{\sqrt{2}}{8}-\frac{1}{8}+\frac{\sqrt{2}}{16} & \frac{1}{8}-\frac{\sqrt{2}}{16}
\end{array}\right) .
$$

We now deform $\Gamma_{s}$ by conjugation to

$$
\Gamma_{\Omega}(x)=\Omega \Gamma_{s}(x) \Omega^{*}, \quad x \in \mathcal{L}\left(G_{8}\right)
$$

where for $r \in G_{8}$,

$$
\Gamma_{\Omega}(\lambda(r))=\sum_{g, h \in \mathbb{Z}_{2}^{2}} \sum_{s, t \in \mathbb{Z}_{2}^{2}} c(g, h) \overline{c(s, t)} \lambda((g, e) r(s, e)) \otimes \lambda((h, e) r(t, e)) .
$$

In view of the properties of $\omega$, it follows that $\mathbb{G}_{8}:=\left(\mathcal{L}\left(G_{8}\right), \Gamma_{\Omega}, \varphi\right)$ is the Kac-Paljutkin algebra, where the (left and right) Haar weight $\varphi$ is the canonical trace on $\mathcal{B}\left(L^{2}\left(G_{8}\right)\right.$ ) 
[22, Example 6.4]. Note that, by construction, $\Gamma_{\Omega}=\Gamma_{s}$ when restricted to the abelian subalgebra $\mathcal{L}\left(\mathbb{Z}_{2} \times \mathbb{Z}_{2}\right)$.

The new co-multiplication also induces a "twisted" pointwise product on $A\left(G_{8}\right)$, given by

$$
(\mu \bullet \nu)(r)=\sum_{g, h \in \mathbb{Z}_{2}^{2}} \sum_{s, t \in \mathbb{Z}_{2}^{2}} c(g, h) \overline{c(s, t)} \mu((g, e) r(s, e)) \nu((h, e) r(t, e))
$$

for $r \in G_{8}, \mu, \nu \in A\left(G_{8}\right)$. Observing that the first row and column of the coefficient matrix (5.7) sum to one while all others sum to zero, it follows easily that the constant function $\mu \equiv 1$ still provides a multiplicative identity for the Banach algebra $L^{1}\left(\mathbb{G}_{8}\right)=$ $\left(A\left(G_{8}\right), \bullet\right)$

By finite dimensionality,

$$
C_{u}\left(\mathbb{G}_{8}\right) \cong C_{0}\left(\mathbb{G}_{8}\right)=L^{\infty}\left(\mathbb{G}_{8}\right)=\mathcal{L}\left(G_{8}\right)
$$

Thus, any $\mu \in \mathcal{P}\left(\mathbb{G}_{8}\right)$ is a positive linear functional on $\mathcal{L}\left(G_{8}\right)$, that is, a positive definite function on $G_{8}$, making $\mathcal{P}\left(\mathbb{G}_{8}\right)=\mathcal{P}\left(G_{8}\right)=\left\{\langle\lambda(\cdot) \xi, \xi\rangle: \xi \in L^{2}\left(G_{8}\right)\right\}$.

Also, by discreteness of $\mathbb{G}_{8}$, we may write the quantum channel $\Theta^{l}(\mu)$, using equation (4.5), as:

$$
\Theta^{l}(\mu)(x)=\left\langle\mu \otimes \iota, \tilde{\Gamma}_{\Omega}(x)\right\rangle=\left\langle\mu \otimes \iota, W_{8}^{*}(1 \otimes x) W_{8}\right\rangle
$$

for all $\mu \in \mathcal{P}_{1}\left(G_{8}\right)$ and $x \in \mathcal{B}\left(L^{2}\left(G_{8}\right)\right)$, where $W_{8}$ is the left fundamental unitary of 
$\mathbb{G}_{8}$, which we now calculate.

Firstly, since the Haar weight $\varphi$ is given by the vector state $\omega_{\delta_{e}}$, we have

$$
\langle\lambda(f), \lambda(g)\rangle_{\varphi}=\omega_{\delta_{e}}\left(\lambda(f) \lambda(g)^{*}\right)=\left(f * g^{*}\right)(e)=\langle f, g\rangle_{L^{2}\left(G_{8}\right)}
$$

for all $f, g \in L^{1}\left(G_{8}\right)$. Consequently, the Hilbert space $H_{\varphi}$ is $L^{2}\left(G_{8}\right)$ via the isometric isomorphism

$$
\Lambda_{\varphi}: H_{\varphi} \ni \lambda(f) \mapsto f \in L^{2}\left(G_{8}\right)
$$

Therefore, for $f, g \in L^{2}\left(G_{8}\right)$, we have

$$
\begin{aligned}
W_{8}^{*}(f \otimes g)= & W_{8}^{*}\left(\Lambda_{\varphi}(\lambda(f)) \otimes \Lambda_{\varphi}(\lambda(g))\right) \\
& \stackrel{(3.1)}{=}\left(\Lambda_{\varphi} \otimes \Lambda_{\varphi}\right)\left(\Gamma_{\Omega}(\lambda(g))(\lambda(f) \otimes 1)\right) \\
= & \sum_{r, r^{\prime} \in G_{8}} g(r) f\left(r^{\prime}\right)\left(\Lambda_{\varphi} \otimes \Lambda_{\varphi}\right)\left(\Gamma_{\Omega}(\lambda(r))\left(\lambda\left(r^{\prime}\right) \otimes 1\right)\right) \\
= & \sum_{r, r^{\prime} \in G_{8}} g(r) f\left(r^{\prime}\right)\left(\Lambda_{\varphi} \otimes \Lambda_{\varphi}\right)\left(\Omega(\lambda(r) \otimes \lambda(r)) \Omega^{*}\left(\lambda\left(r^{\prime}\right) \otimes 1\right)\right) \\
= & \sum_{r, r^{\prime} \in G_{8}} \sum_{x, y \in \mathbb{Z}_{2}^{2}} \sum_{s, t \in \mathbb{Z}_{2}^{2}} g(r) f\left(r^{\prime}\right) c(x, y) \overline{c(s, t)}\left(\delta_{(x, e) r(s, e)} * \delta_{r^{\prime}}\right) \otimes\left(\delta_{(y, e) r(t, e)}\right) \\
= & \sum_{r \in G_{8}} \sum_{x, y \in \mathbb{Z}_{2}^{2}} \sum_{s, t \in \mathbb{Z}_{2}^{2}} g(r) c(x, y) \overline{c(s, t)}\left(\delta_{(x, e)} * \delta_{r} * \delta_{(s, e)} * f\right) \otimes\left(\delta_{(y, e)} * \delta_{r} * \delta_{(t, e)}\right) \\
= & \sum_{r \in G_{8}} \sum_{x, y \in \mathbb{Z}_{2}^{2}} \sum_{s, t \in \mathbb{Z}_{2}^{2}} g(r) c(x, y) \overline{c(s, t)}\left(\lambda(x, e)\left(\delta_{r} * \delta_{(s, e)} * f\right)\right) \otimes\left(\lambda(y, e)\left(\delta_{r} * \delta_{(t, e)}\right)\right)
\end{aligned}
$$

using the left centralizing property of the left regular representation. By definition of the semi-direct product, $r(s, e)=\left(\alpha_{r}(s), e\right) r$ for $s \in \mathbb{Z}_{2}^{2}, r \in G_{8}$, where $\alpha_{r}: \mathbb{Z}_{2}^{2} \rightarrow \mathbb{Z}_{2}^{2}$ 
is the identity, respectively, permutation of coordinates, if the third component of $r \in\left(\mathbb{Z}_{2} \times \mathbb{Z}_{2}\right) \rtimes_{\alpha} \mathbb{Z}_{2}$ is 0 , respectively, 1 . This allows us to write

$$
\delta_{r} * \delta_{(s, e)} * f=\left(\lambda\left(\alpha_{r}(s), e\right) \delta_{r}\right) * f=\lambda\left(\alpha_{r}(s), e\right)\left(\delta_{r} * f\right)
$$

Thus,

$$
W_{8}^{*}(f \otimes g)=\sum_{r \in G_{8}} \sum_{x, y, s, t \in \mathbb{Z}_{2}^{2}} g(r) c(x, y) \overline{c(s, t)} \lambda\left((x, e)\left(\alpha_{r}(s), e\right)\right)\left(\delta_{r} * f\right) \otimes \lambda\left((y, e)\left(\alpha_{r}(t), e\right)\right) \delta_{r}
$$

Noting $\left(\lambda(r) \otimes M_{\delta_{r}}\right)(f \otimes g)=g(r)\left(\delta_{r} * f\right) \otimes \delta_{r}$ for $r \in G_{8}$,

$$
W_{8}^{*}(f \otimes g)=\sum_{r \in G_{8}} \sum_{x, y, s, t \in \mathbb{Z}_{2}^{2}} c(x, y) \overline{c(s, t)}\left(\lambda\left((x, e)\left(\alpha_{r}(s), e\right) r\right) \otimes \lambda\left((y, e)\left(\alpha_{r}(t), e\right)\right) M_{\delta_{r}}\right)(f \otimes g) .
$$

Since $f, g \in L^{2}\left(G_{8}\right)$ were arbitrary, we finally arrive at

$$
W_{8}=\sum_{r \in G_{8}} \sum_{x, y, s, t \in \mathbb{Z}_{2}^{2}} \overline{c(x, y)} c(s, t) \lambda\left(r^{-1}\left(\alpha_{r}(s) x, e\right)\right) \otimes\left(M_{\delta_{r}} \lambda\left(\alpha_{r}(t) y, e\right)\right)
$$

The dual quantum group $\hat{\mathbb{G}}_{8}$ therefore has underlying von Neumann algebra $L^{\infty}\left(\hat{\mathbb{G}}_{8}\right)=$ $\left\{(\mu \otimes \iota)\left(W_{8}\right): \mu \in L^{1}\left(\mathbb{G}_{8}\right)\right\}$.

Now, if $\mu=\omega_{\xi} \in \mathcal{P}_{1}\left(\mathbb{G}_{8}\right)$ for a unit vector $\xi \in L^{2}\left(G_{8}\right)$, then $\Theta^{l}(\mu)$ is the quantum channel on $\mathcal{B}\left(L^{2}\left(G_{8}\right)\right)$ given by

$$
\Theta^{l}(\mu)(x)=\sum_{\imath=1}^{8} W_{\imath}^{*} x W_{\imath}, \quad x \in \mathcal{B}\left(L^{2}\left(G_{8}\right)\right)
$$


where $W_{\imath}=\left(\omega_{\xi, e_{\imath}} \otimes \iota\right)\left(W_{8}\right)$ and $\left(e_{\imath}\right)_{\imath=1}^{8}$ is an orthonormal basis of $L^{2}\left(G_{8}\right)$. More explicitly, the Kraus operators $W_{\imath}$ satisfy

$$
W_{\imath}=\sum_{r \in G_{8}} \sum_{x, y, s, t \in \mathbb{Z}_{2}^{2}} \overline{c(x, y)} c(s, t)\left\langle\lambda\left(r^{-1}\left(\alpha_{r}(s) x, e\right)\right) \xi, e_{\imath}\right\rangle M_{\delta_{r}} \lambda\left(\alpha_{r}(t) y, e\right) .
$$

Note that the pentagonal relation implies that $W_{8}$ is a unitary co-representation of $\hat{\mathbb{G}}_{8}$, making equation (5.10) a concrete realization of the method introduced in §4.3.2.

Since the co-multiplication $\Gamma_{\Omega}$ agrees with $\Gamma_{s}$ on $\mathcal{L}\left(\mathbb{Z}_{2} \times \mathbb{Z}_{2}\right)$, the channels $\Theta^{l}(\mu)$, when restricted to this subalgebra, act as Schur maps coming from the representation (4.2) of $M_{c b} A\left(G_{8}\right)$. However, outside of $\mathcal{L}\left(\mathbb{Z}_{2} \times \mathbb{Z}_{2}\right)$ the channels behave remarkably different. For a simple illustration, consider the characteristic function $\chi_{A}$, where $A:=\{e, z\}=\{(0,0,0),(0,0,1)\} \subseteq G_{8}$. As the characteristic function of a subgroup, $\chi_{A} \in \mathcal{P}_{1}\left(G_{8}\right)$ [34, Example 32.43]. In the co-commutative setting, the quantum channel $\hat{\Theta}\left(\chi_{A}\right)$ has diagonal Kraus operators, and is a projection onto $\mathcal{L}(A) \vee L^{\infty}\left(G_{8}\right)$, its fixed point algebra (§4.4). Hence, $\chi_{A}$ a nonadapted positive definite function in $\mathcal{P}_{1}\left(G_{8}\right)$. On the other hand,

$$
\Theta^{l}\left(\chi_{A}\right)(x)=\sum_{\imath=1}^{4} W_{\imath}^{*} x W_{\imath}
$$

where the Kraus operators have the following matrix representations in the standard 
basis of $L^{2}\left(G_{8}\right)$ (see Appendix):

$$
W_{1}=\left(\begin{array}{cccccccc}
1 & 0 & 0 & 0 & 0 & 0 & 0 & 0 \\
0 & \frac{5}{8} & 0 & \frac{1}{8} & 0 & \frac{1}{8} & 0 & \frac{1}{8} \\
0 & 0 & 0 & 0 & 0 & 0 & 0 & 0 \\
0 & \frac{1}{8} & 0 & \frac{1}{8} & 0 & -\frac{1}{8}-\frac{2}{4} & 0 & -\frac{1}{8}+\frac{2}{4} \\
0 & 0 & 0 & 0 & 0 & 0 & 0 & 0 \\
0 & \frac{1}{8} & 0 & -\frac{1}{8}+\frac{2}{4} & 0 & \frac{1}{8} & 0 & -\frac{1}{8}-\frac{2}{4} \\
0 & 0 & 0 & 0 & 0 & 0 & 0 & 0 \\
0 & \frac{1}{8} & 0 & -\frac{1}{8}-\frac{2}{4} & 0 & -\frac{1}{8}+\frac{2}{4} & 0 & \frac{1}{8}
\end{array}\right) ;
$$




$$
\begin{aligned}
& W_{3}=\left(\begin{array}{cccccccc}
0 & 0 & 0 & 0 & 0 & 0 & 0 & 0 \\
0 & \frac{1}{8} & 0 & \frac{1}{8} & 0 & -\frac{1}{8}+\frac{2}{4} & 0 & -\frac{1}{8}-\frac{2}{4} \\
0 & 0 & 0 & 0 & 0 & 0 & 0 & 0 \\
0 & \frac{1}{8} & 0 & \frac{5}{8} & 0 & \frac{1}{8} & 0 & \frac{1}{8} \\
0 & 0 & 0 & 0 & \frac{1}{8} & 0 & 0 & 0 \\
0 & -\frac{1}{8}-\frac{2}{4} & 0 & \frac{1}{8} & 0 & \frac{1}{8} & 0 & -\frac{1}{8}+\frac{2}{4} \\
0 & 0 & 0 & 0 & 0 & 0 & 0 & 0 \\
0 & -\frac{1}{8}+\frac{2}{4} & 0 & \frac{1}{8} & 0 & -\frac{1}{8}-\frac{2}{4} & 0 & \frac{1}{8}
\end{array}\right) ; \\
& W_{4}=\left(\begin{array}{cccccccc}
0 & 0 & 0 & 0 & 0 & 0 & 0 & 0 \\
0 & \frac{1}{8} & 0 & -\frac{1}{8}+\frac{2}{4} & 0 & -\frac{1}{8}-\frac{2}{4} & 0 & \frac{1}{8} \\
0 & 0 & 0 & 0 & 0 & 0 & 0 & 0 \\
0 & -\frac{1}{8}-\frac{2}{4} & 0 & \frac{1}{8} & 0 & -\frac{1}{8}+\frac{2}{4} & 0 & \frac{1}{8} \\
0 & 0 & 0 & 0 & 0 & 0 & 0 & 0 \\
0 & -\frac{1}{8}+\frac{2}{4} & 0 & -\frac{1}{8}-\frac{2}{4} & 0 & \frac{1}{8} & 0 & \frac{1}{8} \\
0 & 0 & 0 & 0 & 0 & 0 & \frac{1}{8} & 0 \\
0 & \frac{1}{8} & 0 & \frac{1}{8} & 0 & \frac{1}{8} & 0 & \frac{5}{8}
\end{array}\right) .
\end{aligned}
$$

So we see that the Kraus operators of $\Theta^{l}\left(\chi_{A}\right)$ are significantly different from those of its symmetric counterpart.

To calculate the harmonic operators $\mathcal{H}_{\chi_{A}} \subseteq L^{\infty}\left(\mathbb{G}_{8}\right)=\mathcal{L}\left(G_{8}\right)$, we identify $\mathcal{L}\left(G_{8}\right)$ with $L^{2}\left(G_{8}\right)$ via equation (5.8). Under this identification, it follows that 
$\mathcal{B}:=\left\{\frac{1}{\sqrt{8}} \lambda(r): r \in G_{8}\right\}$ forms an orthonormal basis for $L^{2}\left(G_{8}\right)$, and that we may view $\Theta^{l}\left(\chi_{A}\right)$ as an operator in $\mathcal{B}\left(L^{2}\left(G_{8}\right)\right)$ by the invariance condition $\Theta^{l}\left(\chi_{A}\right)\left(\mathcal{L}\left(G_{8}\right)\right) \subseteq$ $\mathcal{L}\left(G_{8}\right)$. The matrix representation of $\Theta^{l}\left(\chi_{A}\right)$ with respect to $\mathcal{B}$ is then given by (see Appendix)

$$
\left[\Theta^{l}\left(\chi_{A}\right)\right]=\left(\begin{array}{cccccccc}
1 & 0 & 0 & 0 & 0 & 0 & 0 & 0 \\
0 & \frac{5}{8} & 0 & \frac{1}{8} & 0 & \frac{1}{8} & 0 & \frac{1}{8} \\
0 & 0 & 0 & 0 & 0 & 0 & 0 & 0 \\
0 & \frac{1}{8} & 0 & \frac{1}{8} & 0 & -\frac{1}{8}-\frac{2}{4} & 0 & -\frac{1}{8}+\frac{2}{4} \\
0 & 0 & 0 & 0 & 0 & 0 & 0 & 0 \\
0 & \frac{1}{8} & 0 & -\frac{1}{8}+\frac{2}{4} & 0 & \frac{1}{8} & 0 & -\frac{1}{8}-\frac{2}{4} \\
0 & 0 & 0 & 0 & 0 & 0 & 0 & 0 \\
0 & \frac{1}{8} & 0 & -\frac{1}{8}-\frac{2}{4} & 0 & -\frac{1}{8}+\frac{2}{4} & 0 & \frac{1}{8}
\end{array}\right) .
$$

Calculating the eigenvalues and eigenvectors of this matrix yields $\mathcal{H}_{X_{A}}=\mathbb{C} I_{8}$. Thus, in contrast to the co-commutative case, the harmonic operators in $L^{\infty}\left(\mathbb{G}_{8}\right)$ are trivial. Moreover, $\tilde{\mathcal{H}}_{\chi_{A}}=L^{\infty}\left(\hat{\mathbb{G}}_{8}\right)^{\prime}$ by Theorem 4.4.4, so $\chi_{A}$ behaves like an "adapted" positive definite functional in $\mathcal{P}_{1}\left(\mathbb{G}_{8}\right)$.

In general, the fixed points of $\Theta^{l}(\mu)$ for $\mu \in \mathcal{P}_{1}\left(\mathbb{G}_{8}\right)$ are the range of a projection $\Theta^{l}(\phi)$ for some idempotent $\phi \in \mathcal{P}_{1}\left(\mathbb{G}_{8}\right)$ satisfying $\phi \bullet \mu=\mu \bullet \phi=\phi[46$, Theorem 5.2]. It is also known that idempotent states on finite-dimensional quantum groups are precisely those $\mu=\omega_{\xi}$ for which $W(\xi \otimes \xi)=\xi \otimes \xi$ [24, Theorem 3.2]. In our 
case, there are 8 solutions to this equation, given by the characteristic functions of the following subgroups of $G_{8}$ (see Appendix):

$$
\begin{gathered}
G_{1}=\left\{e_{8}\right\}, \quad G_{2}=\left\{e_{8}, b\right\}, \quad G_{3}=\left\{e_{8}, a b\right\}, \\
G_{4}=\left\{e_{8}, a b, a z, b z\right\}, \quad G_{5}=\left\{e_{8}, z, a b, a b z\right\}, \\
G_{6}=\left\{e_{8}, a\right\}, \quad G_{7}=\left\{e_{8}, a, b, a b\right\}, \quad G_{8} .
\end{gathered}
$$

Since $G_{i} \subset \mathbb{Z}_{2} \times \mathbb{Z}_{2} \times\{e\}$ for all $i \neq 4,5$, the harmonic operators $\mathcal{H}_{\chi_{G_{i}}}=\mathcal{L}\left(G_{i}\right)$, as in the co-commutative case. For the two remaining groups we have (see Appendix)

$$
\begin{aligned}
& \mathcal{H}_{\chi_{4}}=\operatorname{span}\left\{\lambda\left(e_{8}\right), \lambda(a b),-2 i \lambda(a z)-\lambda(z)+\lambda(a b z), \lambda(a z)+\lambda(b z)\right\} \\
& \mathcal{H}_{\chi_{5}}=\operatorname{span}\left\{\lambda\left(e_{8}\right), \lambda(a b),-2 i \lambda(z)-\lambda(a z)+\lambda(b z), \lambda(z)+\lambda(a b z)\right\} .
\end{aligned}
$$

Noting also that the subgroups $A=\left\{e_{8}, z\right\}$ and $\left\{e_{8}, a b z\right\}$ are missing from this list, we see that the harmonic operators of $\mathbb{G}_{8}$ are quite different from those of its symmetric counterpart.

From the quantum information theory perspective, one gets interesting quantum channels displaying new phenomena. Having already seen that quantum channels coming from $M_{c b} A(G)$ for finite groups $G$ provide intriguing counter-examples to the asymptotic quantum Birkoff conjecture, much more is to be expected for quantum information from quantum channels arising from locally compact quantum groups. 


\section{Bibliography}

[1] C. Anantharaman-Delaroche. On ergodic theorems for free group actions on noncommutative spaces. Probability Theory and Related Fields 135, no. 4, 520-546 (2006).

[2] R. Arens. Operations induced in function classes. Monatshefte fr Mathematik 55, 119 (1951).

[3] A. Arias, A. Gheondea, S. Gudder. Fixed points of quantum operations. Journal of Mathematical Physics 43, no. 12, 58725881 (2002).

[4] S. Baaj, G. Skandalis. Unitaires multiplicatifs et dualité pour les produits croisé de $C^{*}$-algèbres. Annales Scientifiques de l'École Normale Supérieure 26, no. 4, 425-488 (1993).

[5] E. Bédos and L. Tuset. Amenability and co-amenability for locally compact quantum groups. International Journal of Mathematics 14, no.8, 865-884 (2003).

[6] I. Bengtsson and K. Życzkowski. Geometry of Quantum States: An Introduction to Quantum Entanglement. Cambridge University Press (2006).

[7] R. Bhat, V. Pati and V. S. Sunder. On some convex sets and their extreme points. Mathematische Annalen 296, no. 4, 637-648 (1993).

[8] D. P. Blecher and R. R. Smith. The dual of the Haagerup tensor product. Journal of the London Mathemtatical Society (2) 45, no. 1, 126-144 (1992).

[9] O. Bratteli, P.E.T. Jorgensen, A. Kishimoto, and R.F. Werner. Pure states on $\mathcal{O}_{d}$. Journal of Operator Theory 43, no. 1, 97-143 (2000).

[10] G. Birkhoff. Three observations on linear algebra. Universidad Nacional de Tucumán, Revista Serie A 5, 147-151 (1946).

[11] J. de Cannière. U. Haagerup. Multipliers of the Fourier algebras of some simple Lie groups and their discrete subgroups. American Journal of Mathematics 107, no. 2, 455-500 (1985). 
[12] M.-D. Choi and E. G. Effros. Injectivity and operator spaces. Journal of Functional Analysis 24, no. 2, 156-209 (1977).

[13] C. Chou, A.T.-M. Lau and J. Rosenblatt. Approxmation of compact operators by sums of translations. Illonois Journal of Mathematics 29, no. 2, 340-350 (1985).

[14] Chu, C.-H., Lau, A.T.-M. Harmonic Functions on Groups and Fourier Algebras. Lecture Notes in Mathematics 1782, Springer Berlin-Heidelberg-New York (2002).

[15] J. B. Conway. A Course in Operator Theory. Graduate Studies in Mathematics 21, American Mathematical Society, Rhode Island (1991).

[16] J. Crann, M. Neufang. Quantum channels arising from abstract harmonic analysis. Preprint.

[17] M. Daws. Multipliers of locally compact quantum groups via Hilbert $C^{*}$-modules. Journal of the London Mathematical Society, published online August (2011).

[18] M. Daws. Multipliers, self-induced and dual Banach algebras. Dissertationes Mathematicae 470, $62 \mathrm{pp}$ (2010).

[19] M. Daws. Completely positive multipliers of locally compact quantum groups. arXiv:1107.5244.

[20] E. G. Effros, Z.-J. Ruan. Operator Spaces. London Mathematical Society Monographs, New Series 23, Oxford University Press, New York (2000).

[21] M. Enock, J. M Schwartz. Kac Algebras and Duality of Locally Compact Groups. Springer-Verlag, Berlin (1992).

[22] M. Enock, L. Vainerman. Deformation of a Kac algebra by an abelian subgroup. Communications in Mathematical Physics 178, no. 3, 571-596 (1996).

[23] P. Eymard. L'algèbre de Fourier d'un groupe localement compact. Bulletin de la Societé Mathematiques de France 92, 181-236 (1964).

[24] U. Franz and A. Skalski. A new characterization of idempotent states on finite and compact quantum groups. Comptes Rendus Mathematique 347, no. 17-18, 991-996 (2009).

[25] G. B. Folland, A Course in Abstract Harmonic Analysis. Studies in Advanced Mathematics, CRC Press Incorporated, Florida (1995).

[26] F. Ghahramani. Isometric representations of $M(G)$ on $\mathcal{B}(H)$. Glasgow Mathematical Journal 23, no. 2, 119-122 (1982). 
[27] J.E. Gilbert. $L^{p}$-convolution operators of Banach space tensor products I, II, III. Unpublished manuscript.

[28] U. Haagerup. Decomposition of completely bounded maps on operator algebras. Unpublished, Odense University, Denmark, (1980).

[29] U. Haagerup, J. Kraus. Approximation properties for group $C^{*}$-algebras and group von Neumann algebras. Transactions of the American Mathematical Society 344 , no.2, 667-699 (1994).

[30] U. Haagerup, M. Musat. Factorization and dilation problems for completely positive maps on von Neumann algebras. Communications in Mathematical Physics. 303, no. 2, 555-594 (2011).

[31] G. H. Hardy, J. E. Littlewood and G. Pólya. Some simple inequalities satisfied by convex functions. Messenger of Mathematics 58, 145 (1929).

[32] W. Heisenberg. Über quantentheoretishe Umdeutung kinematisher und mechanischer Beziehungen. Zeitschrift fr Physik 33, 879-893, (1925).

[33] J. Helm and W. T. Strunz. Quantum decoherence of two qubits. Physical Review A 80 , no. 4,042108 (2009).

[34] E. Hewitt and K.A. Ross. Abstract Harmonic Analysis II: Structure and Analysis for Compact Groups. Analysis on Locally Compact Abelian Groups. A Series of Comprehensive Studies in Mathematics 152. Springer-Verlag, New York-Berlin (1970).

[35] J. A. Holbrook, D. W. Kribs and R. Laflamme. Noiseless subsystems and the structure of the commutant in quantum error correction. Quantum Information Processing 2, no. 5, 381419 (2004).

[36] Z. Hu, M. Neufang and Z.-J. Ruan. Completely bounded multipliers over locally compact quantum groups. Proceedings of the London Mathematical Society, published online January (2011)

[37] M. Ilie, N. Spronk. Completely bounded homomorphisms of the Fourier algebras. Journal of Functional Analysis 225, no. 2, 480-499 (2005).

[38] M. Izumi. Non-commutative Poisson boundaries and compact quantum group actions. Advances in Mathematics 169, no. 1, 1-57 (2002).

[39] M. Izumi. Non-commutative Poisson boundaries, in: Discrete Geometric Analysis. Contemporary Mathematics 347, American Mathematical Society, Providence, RI 69-81 (2004). 
[40] W. Jaworski, M. Neufang. The Choqet-Deny equatıon in a Banach space. Canadian Journal of Mathematics 59, no. 4, 795827 (2007).

[41] M. Junge, M. Neufang, Z.-J. Ruan. A Representation Theorem for Locally Compact Quantum Groups. International Journal of Mathematics 20, no. 3, 377-400 (2009).

[42] G. I. Kac and V. G.Paljutkin. An example of a ring group of order eight. Russian Mathematical Surveys 20, 268-269 (1965).

[43] R. V. Kadison, J. R. Ringrose. Fundamentals of the Theory of Operator Algebras, Volume I: Elementary Theory. Academic Press Incorporated (1983).

[44] R. V. Kadison, J. R. Ringrose. Fundamentals of the Theory of Operator Algebras, Volume II: Advanced Theory. Academic Press Incorporated (1983).

[45] V. A. Kaimanovich, A. M. Vershik. Random walks on discrete groups: boundary and entropy. The Annals of Probability 11, no 3, 457-490 (1983).

[46] M. Kalantar, M. Neufang, Z.-J. Ruan. Non-commutatıve Pousson boundarzes over locally compact quantum groups. Preprint (2011).

[47] D. W. Kribs. Quantum channels, wavelets, dilatıons, and representatıons of $O_{n}$. Proceedings of the Edinburgh Mathematical Society, 46, no.2, 421-433 (2003).

[48] J. Kustermans. Locally compact quantum groups in the unvversal settrng. International Journal of Mathematics 12, no. 3, 289338 (2001).

[49] J. Kustermans. One-parameter representatıons on $C^{*}$-algebras. Preprint Odense Universitet (1997).

[50] J. Kustermans, S. Vaes. Locally compact quantum groups. Annales Scientifiques de l'École Normale Supérieure 33, no. 6, 837-934 (2000).

[51] J. Kustermans, S. Vaes. Locally compact quantum groups in the von Neumann algebrauc settıng. Mathematica Scandinavica 92, no. 1, 68-92 (2003).

[52] R. Laflamme. Private communication.

[53] L. J. Landau and R. F. Streater. On Birkoff's theorem for doubly stochastic completely positive maps of matrix algebras. Linear Algebra and its Applications 193, 107-127 (1993).

[54] C.-K. Li, B.-S. Tam, A note on extreme correlatıon matrices. SIAM Journal on Matrix Analysis and Applications 15, no. 3, 903-908 (1994).

[55] B. J. Lim. Noncommutative Porsson boundaries of unital quantum channels. Journal of Mathematical Physics 51, 052202 (2010). 
[56] A. Mukherjea. Limit theorems for probability measures on non-compact groups and semigroups. Z. Wahrscheinlichkeitstheorie Verw. Gebiete 33, no. 4 273-284 (1976).

[57] M. Neufang. Abstrakte Harmonische Analyse und Modulhomomorphismen 'u von Neumann-Algebren. Ph.D. Thesis at University of Saarland, Saarbr'ucken, Germany (2000).

[58] M. Neufang, Z.-J. Ruan, N. Spronk. Completely isometric representations of $M_{c b} A(G)$ and $U C B(\hat{G})^{*}$. Transactions of the American Mathematical Society 360, no. 3, 1133-1161 (2008).

[59] M. Neufang, V. Runde. Harmonic operators: the dual perspective. Mathematische Zeitschrift 255, no. 3, 669-690 (2007).

[60] M. A. Nielsen and I. L. Chuang. Quantum Computation and Quantum Information. Cambridge University Press (2000).

[61] V. Paulsen. Completely Bounded Maps and Operator Algebras. Cambridge University Press (2002).

[62] L.-B. Ren. Introduction to Operator Algebras. World Scientific Publishing Co. Pte. Ltd. (1992).

[63] Z.-J. Ruan. Operator amenability of $A(G)$. American Journal of Mathematics 117 , no. 6,14491474 (1995).

[64] Z.-J. Ruan. On the predual of dual algebras. Journal of Operator Theory 27, no. 1, 179-192 (1992).

[65] Z.-J. Ruan. Injectivity of operator spaces. Transactions of the American Mathematical Society 315, no. 1, 89-104, (1989).

[66] S. Sakai. A characterization of $W^{*}$-algebras. Pacific Journal of Mathematics 6, 763-773 (1956).

[67] J. Schur. Über eine Klasse von Mittelbildungen mit Anwendungen auf die Determinantentheorie. Sitzungsberichte Berlin Mathematischen Gesellschaft 22, no. 9 (1923).

[68] N. Spronk. Measurable schur multipliers and completely bounded multipliers of the Fourier algebras. Proceedings of the London Mathematical Society 89, no. 1, 161-192 (2004).

[69] W. F. Stinespring. Positive functions on $C^{*}$-algebras. Proceedings of the American Mathematical Society 6, no. 2, 211-216 (1955). 
[70] M. H. Stone. Linear transformations in Hilbert space, III: operational methods and group theory. Proceedings of the National Academy of Sciences U.S.A. 16, $172-175(1930)$.

[71] E. Størmer, Regular abelian Banach algebras of linear maps of operator algebras. Journal of Functional Analysis 37, 331-373 (1980).

[72] M. Takesaki. A characterization of group algebras as a converse of TannakaStinespringTatsuuma duality theorem. American Journal of Mathematics 91, 529564 (1969).

[73] M. Takesaki. Theory of Operator Algebras I. Encyclopedia of Mathematical Sciences 124, Springer-Verlag Berlin-Heidelberg-New York (2002).

[74] M. Takesaki. Theory of Operator Algebras II. Encyclopedia of Mathematical Sciences 125, Springer-Verlag Berlin-Heidelberg-New York (2003).

[75] J. Tomiyama. Tensor products and properties of norm one in von Neumann algebras. Lecture Notes, University of Copenghagen (1970)

[76] S. Vaes. Locally Compact Quantum Groups. Ph.D. thesis, K.U. Leuven (2000).

[77] S. Vaes, L. Vainerman. On low-dimensional locally compact quantum groups. Locally Compact Quantum Groups and Groupoids. Proceedings of the Meeting of Theoretical Physicists and Mathematicians, Strasbourg, February 21 - 23 (2002).

[78] S. Vaes and A. Van Daele. The Heisenberg commutation relations, commuting squares and the Haar measure on locally compact quantum groups. Operator Algebras and Mathematical Physics 379-400 (Constanta, 2001) (Theta, Bucharest, 2003).

[79] A. Van Daele. Locally compact quantum groups. A von Neumann algebra approach. preprint: zrXiv:math/0602212v1 (2006).

[80] J. von Neumann. Die Eindeutigkeit der Schr'odingerschen Operatoren. Mathematische Annalen 104, 570-578 (1931).

[81] A. Weil. Lintégration dans les groupes topologiques et ses applications. Actualités Scientifiques et Industrielles, 869 Paris (1940).

[82] J. G. Wendel. Left centralizers and isomorphisms of group algebras. Pacific Journal of Mathematics 2, 251-261 (1952).

[83] S. L. Woronowicz. Twisted SU(2) group. An example of a non-commutative differential calculus. Publications of the Research Institute for Mathematical Sciences, Kyoto University 23, no. 1, 117-181 (1987). 
[84] S. L. Woronowicz. From multiplicative unitaries to quantum groups. International Journal of Mathematics 7, no. 1, 127149 (1996).

[85] V. Zarikian. Alternating-projection algorithms for operator-theoretic calculations. Linear Algebra and its Applications 419, 2-3, 710-734 (2006). 


\section{Appendix}

Below is the Maple code used to perform the various linear algebra calculations in $\S 5.3$ of the thesis.

We begin with a calculation of the coefficient matrix (5.7). The function $\omega \in$ $L^{\infty}\left(\hat{\mathbb{Z}}_{2}{ }^{2} \times \hat{\mathbb{Z}}_{2}^{2}\right)$ is stored, and an array $\Phi$ is defined to keep track of the various products of characters of $\mathbb{Z}_{2} \times \mathbb{Z}_{2}$ in order to implement the Fourier transform.

restart;

with(LinearAlgebra):

$\mathrm{w}:=\operatorname{Array}(0 . .3,0 . .3)$ :

$\mathrm{w}[0,0]:=1: \mathrm{w}[0,1]:=1: \mathrm{w}[0,2]:=1: \mathrm{w}[0,3]:=1:$

$\mathrm{w}[1,0]:=1: \mathrm{w}[1,1]:=1: \mathrm{w}[1,2]:=\exp \left(\mathrm{I}^{*} \mathrm{Pi} / 4\right): \mathrm{w}[1,3]:=\exp \left(-\mathrm{I}^{*} \mathrm{Pi} / 4\right):$

$\mathrm{w}[2,0]:=1: \mathrm{w}[2,1]:=\exp \left(-\mathrm{I}^{*} \mathrm{Pi} / 4\right): \mathrm{w}[2,2]:=1: \mathrm{w}[2,3]:=\exp \left(\mathrm{I}^{*} \mathrm{Pi} / 4\right):$

$\mathrm{w}[3,0]:=1: \mathrm{w}[3,1]:=\exp \left(\mathrm{I}^{*} \mathrm{Pi} / 4\right): \mathrm{w}[3,2]:=\exp \left(-\mathrm{I}^{*} \mathrm{Pi} / 4\right): \mathrm{w}[3,3]:=1$ :

phi $:=\operatorname{Array}(0 . .1,0 . .1)$ :

phi $[0,0]:=1: \operatorname{phi}[0,1]:=1$ :

phi $[1,0]:=1$ : phi $[1,1]:=-1$ :

Phi $:=\operatorname{Array}(0 . .3,0 . .3,0 . .3,0 . .3)$ :

for i from 0 by 1 to 3 do

for $\mathrm{j}$ from 0 by 1 to 3 do

$\operatorname{Phi}[0,0, i, j]:=1$;

end do:

end do:

for i from 0 by 1 to 3 do

for $\mathrm{j}$ from 0 by 1 to 3 do

if $\operatorname{modp}(\mathrm{j}, 2)=1$ then $\operatorname{Phi}[0,1, \mathrm{i}, \mathrm{j}]:=-1$

else Phi $[0,1, \mathrm{i}, \mathrm{j}]:=1$

end if:

end do:

end do:

for i from 0 by 1 to 3 do

for $\mathrm{j}$ from 0 by 1 to 3 do

if $\mathrm{j} 1$ then $\operatorname{Phi}[0,2, \mathrm{i}, \mathrm{j}]:=-1$

else $\operatorname{Phi}[0,2, \mathrm{i}, \mathrm{j}]:=1$ 
end if:

end do:

end do:

for i from 0 by 1 to 3 do

for $\mathrm{j}$ from 0 by 1 to 3 do

if $\operatorname{modp}(\mathrm{j}, 3)=0$ then $\operatorname{Phi}[0,3, \mathrm{i}, \mathrm{j}]:=1$

else $\operatorname{Phi}[0,3, \mathrm{i}, \mathrm{j}]:=-1$

end if:

end do:

end do:

for $\mathrm{k}$ from 0 by 1 to 3 do

for i from 0 by 1 to 3 do

for $\mathrm{j}$ from 0 by 1 to 3 do

if $\operatorname{modp}(\mathrm{i}, 2)=1$ then $\operatorname{Phi}[1, \mathrm{k}, \mathrm{i}, \mathrm{j}]:=-1 * \operatorname{Phi}[0, \mathrm{k}, \mathrm{i}, \mathrm{j}]$

else Phi $[1, k, i, j]:=\operatorname{Phi}[0, k, i, j]$

end if:

end do:

end do:

end do:

for $\mathrm{k}$ from 0 by 1 to 3 do

for i from 0 by 1 to 3 do

for $\mathrm{j}$ from 0 by 1 to 3 do

if i1 then $\mathrm{Phi}[2, \mathrm{k}, \mathrm{i}, \mathrm{j}]:=-1 * \mathrm{Phi}[0, \mathrm{k}, \mathrm{i}, \mathrm{j}]$

else Phi $[2, k, i, j]:=P h i[0, k, i, j]$

end if:

end do:

end do:

end do:

for $\mathrm{k}$ from 0 by 1 to 3 do

for $i$ from 0 by 1 to 3 do

for $\mathrm{j}$ from 0 by 1 to 3 do

if $\operatorname{modp}(\mathrm{i}, 3)=0$ then $\mathrm{Phi}[3, \mathrm{k}, \mathrm{i}, \mathrm{j}]:=\mathrm{Phi}[0, \mathrm{k}, \mathrm{i}, \mathrm{j}]$

else $\mathrm{Phi}[3, \mathrm{k}, \mathrm{i}, \mathrm{j}]:=-1 * \mathrm{Phi}[0, \mathrm{k}, \mathrm{i}, \mathrm{j}]$

end if:

end do:

end do:

end do:

$c:=\operatorname{Array}(0 . .3,0 . .3)$ :

for i from 0 by 1 to 3 do

for $\mathrm{j}$ from 0 by 1 to 3 do

for $\mathrm{k}$ from 0 by 1 to 3 do

for 1 from 0 by 1 to 3 do 
$c[i, j]:=c[i, j]+w[k, l] * \operatorname{Phi}[k, l, i, j]$

end do:

end do:

end do:

end do:

for i from 0 by 1 to 3 do

for $\mathrm{j}$ from 0 by 1 to 3 do

$c[i, j]:=1 / 16^{*} c[i, j]$

end do:

end do:

Next, to calculate the Kraus operators of $\Theta^{l}\left(\chi_{A}\right)$, we define the appropriate arrays corresponding to the left regular representation, the standard basis in $\mathbb{C}^{8}$, and the multiplication operators of $\delta_{s}, s \in G_{8}$. We also store the group multiplication tables of $\mathbb{Z}_{2}$ and $\mathbb{Z}_{2}^{2}$ in arrays $\alpha$ and $\beta$, respectively. We then define the appropriate arrays to split the computation into a few steps.

lambda: $=\operatorname{Array}(0 . .7)$ :

lambda $[0]:=$ IdentityMatrix $(8)$ :

lambda[1] := Matrix $([[0,1,0,0,0,0,0,0],[1,0,0,0,0,0,0,0]$,

$[0,0,0,0,0,1,0,0],[0,0,0,0,1,0,0,0],[0,0,0,1,0,0,0,0]$,

$[0,0,1,0,0,0,0,0],[0,0,0,0,0,0,0,1],[0,0,0,0,0,0,1,0]])$ :

lambda $[2]:=$ Matrix $([[0,0,1,0,0,0,0,0],[0,0,0,1,0,0,0,0]$, $[1,0,0,0,0,0,0,0],[0,1,0,0,0,0,0,0],[0,0,0,0,0,0,1,0]$, $[0,0,0,0,0,0,0,1],[0,0,0,0,1,0,0,0],[0,0,0,0,0,1,0,0]])$ :

lambda[3] := lambda[2].lambda[1]:

lambda[4] := Matrix $([[0,0,0,0,1,0,0,0],[0,0,0,0,0,1,0,0]$, $[0,0,0,0,0,0,1,0],[0,0,0,0,0,0,0,1],[1,0,0,0,0,0,0,0]$, $[0,1,0,0,0,0,0,0],[0,0,1,0,0,0,0,0],[0,0,0,1,0,0,0,0]]):$

lambda[5] := lambda[4].lambda[1]:

lambda[6]:= lambda[2].lambda[4]:

lambda[7] := lambda[2].lambda[4].lambda[1]:

$\mathrm{e}:=\operatorname{Array}(0 . .7)$ :

$\mathrm{e}[0]:=\operatorname{Vector}([1,0,0,0,0,0,0,0])$ :

$\mathrm{e}[1]:=\operatorname{Vector}([0,1,0,0,0,0,0,0])$ :

$\mathrm{e}[2]:=\operatorname{Vector}([0,0,1,0,0,0,0,0])$ :

$\mathrm{e}[3]:=\operatorname{Vector}([0,0,0,1,0,0,0,0])$ :

$\mathrm{e}[4]:=\operatorname{Vector}([0,0,0,0,1,0,0,0])$ :

$\mathrm{e}[5]:=\operatorname{Vector}([0,0,0,0,0,1,0,0])$ :

e[6]:=Vector $([0,0,0,0,0,0,1,0])$ : 
$\mathrm{e}[7]:=\operatorname{Vector}([0,0,0,0,0,0,0,1]):$

M:Array $(0 . .7)$ :

$\mathrm{M}[0]:=\operatorname{Matrix}([[1,0,0,0,0,0,0,0],[0,0,0,0,0,0,0,0]$, $[0,0,0,0,0,0,0,0],[0,0,0,0,0,0,0,0],[0,0,0,0,0,0,0,0]$, $[0,0,0,0,0,0,0,0],[0,0,0,0,0,0,0,0],[0,0,0,0,0,0,0,0]])$ :

$\mathrm{M}[1]:=\operatorname{Matrix}([[0,0,0,0,0,0,0,0],[0,1,0,0,0,0,0,0]$, $[0,0,0,0,0,0,0,0],[0,0,0,0,0,0,0,0],[0,0,0,0,0,0,0,0]$, $[0,0,0,0,0,0,0,0],[0,0,0,0,0,0,0,0],[0,0,0,0,0,0,0,0]])$ :

$\mathrm{M}[2]:=$ Matrix $([[0,0,0,0,0,0,0,0],[0,0,0,0,0,0,0,0]$, $[0,0,1,0,0,0,0,0],[0,0,0,0,0,0,0,0],[0,0,0,0,0,0,0,0]$, $[0,0,0,0,0,0,0,0],[0,0,0,0,0,0,0,0],[0,0,0,0,0,0,0,0]])$ :

$\mathrm{M}[3]:=\operatorname{Matrix}([[0,0,0,0,0,0,0,0],[0,0,0,0,0,0,0,0]$, $[0,0,0,0,0,0,0,0],[0,0,0,1,0,0,0,0],[0,0,0,0,0,0,0,0]$, $[0,0,0,0,0,0,0,0],[0,0,0,0,0,0,0,0],[0,0,0,0,0,0,0,0]])$ :

$\mathrm{M}[4]:=\operatorname{Matrix}([[0,0,0,0,0,0,0,0],[0,0,0,0,0,0,0,0]$, $[0,0,0,0,0,0,0,0],[0,0,0,0,0,0,0,0],[0,0,0,0,1,0,0,0]$, $[0,0,0,0,0,0,0,0],[0,0,0,0,0,0,0,0],[0,0,0,0,0,0,0,0]])$ :

$\mathrm{M}[5]:=\operatorname{Matrix}([[0,0,0,0,0,0,0,0],[0,0,0,0,0,0,0,0]$, $[0,0,0,0,0,0,0,0],[0,0,0,0,0,0,0,0],[0,0,0,0,0,0,0,0]$, $[0,0,0,0,0,1,0,0],[0,0,0,0,0,0,0,0],[0,0,0,0,0,0,0,0]])$ :

$\mathrm{M}[6]:=\operatorname{Matrix}([[0,0,0,0,0,0,0,0],[0,0,0,0,0,0,0,0]$, $[0,0,0,0,0,0,0,0],[0,0,0,0,0,0,0,0],[0,0,0,0,0,0,0,0]$, $[0,0,0,0,0,0,0,0],[0,0,0,0,0,0,1,0],[0,0,0,0,0,0,0,0]])$ :

$\mathrm{M}[7]:=\operatorname{Matrix}([[0,0,0,0,0,0,0,0],[0,0,0,0,0,0,0,0]$, $[0,0,0,0,0,0,0,0],[0,0,0,0,0,0,0,0],[0,0,0,0,0,0,0,0]$, $[0,0,0,0,0,0,0,0],[0,0,0,0,0,0,0,0],[0,0,0,0,0,0,0,1]])$ :

lambdah: $=\operatorname{Array}(0 . .3):$

for i from 0 to 3 do lambdah $[\mathrm{i}]:=$ conjugate $(\mathrm{c}[0, \mathrm{i}]) *$ lambda $[0]+$ conjugate $(\mathrm{c}[1, \mathrm{i}])^{*}$ lambda $[2]+$ conjugate $(\mathrm{c}[2, \mathrm{i}]) *$ lambda $[4]+$ conjugate $(\mathrm{c}[3, \mathrm{i}])^{*}$ lambda $[6]$ :

end do:

alpha: $=\operatorname{Array}(0 . .3)$ :

alpha[0]: $=0$ :

alpha $[1]:=2$ : 
alpha[2]:=1:

alpha $[3]:=3$ :

beta: $=\operatorname{Array}(0 . .3,0 . .3)$ :

$\operatorname{beta}[0,0]:=0: \operatorname{beta}[0,1]:=1: \operatorname{beta}[0,2]:=2: \operatorname{beta}[0,3]:=3$ :

$\operatorname{beta}[1,0]:=1$ : $\operatorname{beta}[1,1]:=0: \operatorname{beta}[1,2]:=3$ : beta $[1,3]:=2$ :

$\operatorname{beta}[2,0]:=2: \operatorname{beta}[2,1]:=3: \operatorname{beta}[2,2]:=0: \operatorname{beta}[2,3]:=1$ :

$\operatorname{beta}[3,0]:=3: \operatorname{beta}[3,1]:=2: \operatorname{beta}[3,2]:=1$ : $\operatorname{beta}[3,3]:=0$ :

$\mathrm{v}:=\operatorname{Vector}([1,1,0,0,0,0,0,0])$ :

$\mathrm{xi}:=\mathrm{v} / \operatorname{VectorNorm}(\mathrm{v}, 2$, conjugate $=$ true $) ;$

$\mathrm{d}:=\operatorname{Array}(0 . .7,0 . .7,0 . .3,0 . .3)$ :

for i from 0 by 1 to 7 do

for $\mathrm{x}$ from 0 by 1 to 7 do

for t from 0 by 1 to 3 do

for h from 0 by 1 to 3 do

for $\mathrm{s}$ from 0 to 3 do

if $\operatorname{modp}(\mathrm{x}, 2)=1$ then

$\mathrm{d}[\mathrm{i}, \mathrm{x}, \mathrm{t}, \mathrm{h}]:=\mathrm{d}[\mathrm{i}, \mathrm{x}, \mathrm{t}, \mathrm{h}]+$ simplify

$(\mathrm{c}[\mathrm{s}, \mathrm{t}] *$ DotProduct $(\mathrm{e}[\mathrm{i}]$, HermitianTranspose

(lambda[x]).lambda [2*alpha[s]].lambdah[h].xi)):

else

$\mathrm{d}[\mathrm{i}, \mathrm{x}, \mathrm{t}, \mathrm{h}]:=\mathrm{d}[\mathrm{i}, \mathrm{x}, \mathrm{t}, \mathrm{h}]+$ simplify

(c[s,t $]^{*}$ DotProduct $(\mathrm{e}[\mathrm{i}]$, HermitianTranspose

(lambda[x]).lambda[2*s].lambdah[h].xi)):

end if:

end do:

end do:

end do:

end do:

end do:

$\mathrm{W}:=\operatorname{Array}(0 . .7)$ :

for $\mathrm{i}$ from 0 by 1 to 7 do

for $\mathrm{x}$ from 0 by 1 to 7 do

for $\mathrm{t}$ from 0 by 1 to 3 do

for $\mathrm{h}$ from 0 by 1 to 3 do

if $\operatorname{modp}(\mathrm{x}, 2)=1$ then

$\mathrm{W}[\mathrm{i}]:=\mathrm{W}[\mathrm{i}]+\operatorname{simplify}\left(\mathrm{d}[\mathrm{i}, \mathrm{x}, \mathrm{t}, \mathrm{h}]^{*} \mathrm{M}[\mathrm{x}]\right.$

.lambda[2*alpha[t]].lambda[2*h]):

else

$\mathrm{W}[\mathrm{i}]:=\mathrm{W}[\mathrm{i}]+\operatorname{simplify}\left(\mathrm{d}[\mathrm{i}, \mathrm{x}, \mathrm{t}, \mathrm{h}]^{*} \mathrm{M}[\mathrm{x}]\right.$

.lambda[2*t].lambda[2*h]):

end if:

end do: 
end do:

end do:

end do:

To calculate the matrix representation of $\Theta^{l}(\mu)$ in the Hilbert-Schmidt basis of $\mathcal{L}\left(G_{8}\right)$, we simply use the standard formulas and define the appropriate arrays to split to computation into a few steps. By changing the input vector from $(1,1,0,0,0,0,0,0)$ to $(1,0,0,1,0,1,1,0)$ and $(1,1,0,0,0,0,1,1)$, one gets the Hilbert-Schmidt matrix for the two idempotents giving rise to the nontrivial harmonic functions $\mathcal{H}_{\chi_{G_{2}}}, i=4,5$. The eigenvalues and eigenvectors may then be computed directly.

$\mathrm{s}:=\operatorname{Array}(0 . .3,0 . .3,0 . .3,0 . .3)$ :

for $\mathrm{r}$ from 0 by 1 to 3 do

for $\mathrm{t}$ from 0 by 1 to 3 do

for i from 0 by 1 to 3 do

for $\mathrm{j}$ from 0 by 1 to 3 do

for $\mathrm{k}$ from 0 by 1 to 3 do

$\mathrm{s}[\mathrm{r}, \mathrm{t}, \mathrm{i}, \mathrm{j}]:=\mathrm{s}[\mathrm{r}, \mathrm{t}, \mathrm{i}, \mathrm{j}]+\mathrm{c}[\mathrm{k}, \text { beta}[\text { alpha[j], beta }[\mathrm{r}, \mathrm{t}]]]^{*}$ lambda $[2 *$ beta[k,beta[alpha[i], $\left.\left.\mathrm{r}]\right]+1\right]$ end do;

end do;

end do;

end do;

end do;

$\mathrm{S}:=\operatorname{Array}(0 . .3,0 . .3)$ :

for $\mathrm{r}$ from 0 by 1 to 3 do

for $\mathrm{t}$ from 0 by 1 to 3 do

for i from 0 by 1 to 3 do

for $\mathrm{j}$ from 0 by 1 to 3 do

$\mathrm{S}[\mathrm{r}, \mathrm{t}]:=\mathrm{S}[\mathrm{r}, \mathrm{t}]+\operatorname{simplify}($ conjugate $(c[\mathrm{i}, \mathrm{j}]) * \mathrm{~s}[\mathrm{r}, \mathrm{t}, \mathrm{i}, \mathrm{j}])$

end do:

end do:

end do:

end do:

$\mathrm{v} 2:=\operatorname{Vector}([1,1,0,0,0,0,0,0])$ :

$\mathrm{xi} 2:=\mathrm{v} 2 /$ VectorNorm $(\mathrm{v} 2,2$, conjugate $=$ true $)$;

$\mathrm{HS}:=\operatorname{Array}(0 . .7,0 . .7)$ :

for $\mathrm{r}$ from 0 to $7 \mathrm{do}$

for $\mathrm{t}$ from 0 to $7 \mathrm{do}$

if $\operatorname{modp}(\mathrm{r}, 2)=1$ then

if $\operatorname{modp}(\mathrm{t}, 2)=1$ then

$\mathrm{HS}[\mathrm{r}, \mathrm{t}]:=\operatorname{simplify}(\operatorname{DotProduct}(\mathrm{xi} 2, \mathrm{~S}[(\mathrm{t}-1) / 2,(\mathrm{r}-1) / 2] \cdot \mathrm{xi} 2))$

else

$\mathrm{HS}[\mathrm{r}, \mathrm{t}]:=0$ : 
end if:

else

if $\mathrm{t}=\mathrm{r}$ then

HS $[r, t]:=$ simplify(DotProduct(xi2,lambda $[r] . x i 2))$

else

$\mathrm{HS}[\mathrm{r}, \mathrm{t}]:=0$

end if:

end if:

end do:

end do:

HSM:=Matrix $([[\operatorname{HS}[0,0], \operatorname{HS}[0,1], \operatorname{HS}[0,2], \operatorname{HS}[0,3], \operatorname{HS}[0,4], \operatorname{HS}[0,5], \operatorname{HS}[0,6], \operatorname{HS}[0,7]]$

,[HS[1,0],HS[1,1],HS[1,2],HS[1,3],HS[1,4],HS[1,5],HS[1,6],HS[1,7]]

,[HS[2,0],HS[2,1],HS[2,2],HS[2,3],HS[2,4],HS[2,5],HS[2,6],HS[2,7]]

,$[\mathrm{HS}[3,0], \mathrm{HS}[3,1], \mathrm{HS}[3,2], \mathrm{HS}[3,3], \mathrm{HS}[3,4], \mathrm{HS}[3,5], \mathrm{HS}[3,6], \mathrm{HS}[3,7]]$

,$[\mathrm{HS}[4,0], \mathrm{HS}[4,1], \mathrm{HS}[4,2], \mathrm{HS}[4,3], \mathrm{HS}[4,4], \mathrm{HS}[4,5], \mathrm{HS}[4,6], \mathrm{HS}[4,7]]$

,$[\mathrm{HS}[5,0], \mathrm{HS}[5,1], \mathrm{HS}[5,2], \mathrm{HS}[5,3], \mathrm{HS}[5,4], \mathrm{HS}[5,5], \mathrm{HS}[5,6], \mathrm{HS}[5,7]]$

,$[\mathrm{HS}[6,0], \mathrm{HS}[6,1], \mathrm{HS}[6,2], \mathrm{HS}[6,3], \mathrm{HS}[6,4], \mathrm{HS}[6,5], \mathrm{HS}[6,6], \mathrm{HS}[6,7]]$

$,[\operatorname{HS}[7,0], \mathrm{HS}[7,1], \mathrm{HS}[7,2], \mathrm{HS}[7,3], \mathrm{HS}[7,4], \mathrm{HS}[7,5], \mathrm{HS}[7,6], \mathrm{HS}[7,7]]])$;

Eigenvectors(HSM);

Finally, to solve for the idempotents, we need a function called KroneckerProduct which computes the Kronecker product of two matrices/vectors. One then stores the fundamental unitary $W_{8}$ in a few steps and solves the fixed point equation $W_{8}(\xi \otimes \xi)=$ $\xi \otimes \xi$.

KroneckerProduct:=proc(A::Matrix,B::Matrix)

local $\mathrm{m}, \mathrm{n}$ :

$\mathrm{m}, \mathrm{n}:=$ LinearAlgebra:-Dimensions(A):

$\operatorname{Matrix}([\operatorname{seq}([\operatorname{seq}(A[j, i] * B, i=1 . . n)], j=1 . . m)])$

end proc:

interface $($ rtablesize $=$ infinity $)$ :

$\mathrm{B}:=$ Matrix $(2,3$, symbol $=\mathrm{b})$;

$\mathrm{A}:=$ Matrix $(2,2$, symbol $=\mathrm{a})$;

simplify(KroneckerProduct(A,B));

Omega:=ZeroMatrix (64):

for i from 0 to 3 do

for $\mathrm{j}$ from 0 to 3 do

Omega: = Omega $+\operatorname{simplify}\left(\mathrm{c}[\mathrm{i}, \mathrm{j}]{ }^{*}\right.$ KroneckerProduct

(lambda[2*i],lambda[2*j]))

end do:

end do:

W8i:=ZeroMatrix(64): 
for $\mathrm{x}$ from 0 to 7 do

for $\mathrm{k}$ from 0 to 3 do

for 1 from 0 to 3 do

if $\operatorname{modp}(\mathrm{x}, 2)=0$ then

W8i: $=$ W8i $+\operatorname{simplify}(c[k, l]]^{*}$

KroneckerProduct

(HermitianTranspose(lambda $[\mathrm{x}]$ ).

lambda $[2 * \mathrm{k}], \mathrm{M}[\mathrm{x}]$. lambda $[2 * 1]))$

else

W8i: $=$ W8i+simplify $\left(c[k, 1]^{*}\right.$

KroneckerProduct

(HermitianTranspose(lambda[x]).

lambda $[2 *$ alpha $[\mathrm{k}]], \mathrm{M}[\mathrm{x}]$. lambda[2*alpha[l]]))

end if:

end do:

end do:

$\operatorname{print}(\mathrm{x})$;

end do:

W8:=simplify(W8i.HermitianTranspose(Omega)):

Eta: $=\operatorname{Vector}(8$, symbol=eta $)$ :

Etan: $=$ Eta/VectorNorm $($ Eta,2,conjugate $=$ true):

EtaM:=Matrix $([[\operatorname{eta}[1]],, \operatorname{eta}[2]],[\operatorname{eta}[3]],[\operatorname{eta}[4]],, \operatorname{eta}[5]]$,

[eta[6]], [eta[7]],,[eta[8]]]);

cEtaM:=convert(KroneckerProduct(EtaM,EtaM),Vector):

LinearSolve(W8, convert(KroneckerProduct(EtaM,EtaM),Vector)):

Bas: $=\operatorname{Array}(0 . .63)$ :

for i from 0 to 63 do

$\operatorname{Bas}[\mathrm{i}]:=\operatorname{Vector}(64)$;

for $\mathrm{j}$ from 1 to 64 do

if $\mathrm{j}=\mathrm{i}+1$ then $\operatorname{Bas}[\mathrm{i}][\mathrm{j}]:=1$

else

$\operatorname{Bas}[\mathrm{i}][\mathrm{j}]:=0$

end if:

end do:

end do:

eq:=Array $(0 . .63)$ :

for i from 0 to 63 do

eq[i]:=simplify(DotProduct(Bas[i],(W8.cEtaM - cEtaM)))

end do:

¿ solve $($ eq $[0]=0$, eq $[1]=0$, eq $[2]=0$, eq $[3]=0$,

eq $[4]=0$, eq $[5]=0$, eq $[6]=0$, eq $[7]=0$,

eq $[8]=0$, eq $[9]=0$, eq $[10]=0$, eq $[11]=0$, eq $[12]=0$, 
eq $[13]=0$, eq $[14]=0$, eq $[15]=0$,

eq[16] $=0$,eq $[17]=0$, eq $[18]=0$,eq $[19]=0$,eq $[20]=0$,

eq $[21]=0$, eq $[22]=0$,eq[23] $=0$,

eq $[24]=0$, eq $[25]=0$,eq $[26]=0$, eq $[27]=0$,eq $[28]=0$,

eq $[29]=0$, eq $[30]=0$, eq $[31]=0$,

eq $[32]=0$, eq $[33]=0$, eq $[34]=0$,eq $[35]=0$, eq $[36]=0$,

eq $[37]=0$,eq $[38]=0$,eq $[39]=0$,

eq $[40]=0$, eq $[41]=0$, eq $[42]=0$, eq $[43]=0$, eq $[44]=0$,

eq $[45]=0$, eq $[46]=0$,eq $[47]=0$,

eq $[48]=0$, eq $[49]=0$, eq $[50]=0$, eq $[51]=0$, eq $[52]=0$,

eq $[53]=0$, eq $[54]=0$,eq $[55]=0$,

eq $[56]=0$,eq $[57]=0$, eq $[58]=0$, eq $[59]=0$, eq $[60]=0$,

eq $[61]=0$, eq $[62]=0$,eq $[63]=0$ 